\title{
Uso do modelo Slash Dobrado em dados de longa duração
}

\author{
Marcos Rafael Nogueira Cavalcante
}

TESE APRESENTADA

$\mathrm{AO}$

Instituto DE MATEMÁticA E EstatísticA

DA

Universidade DE SÃo PAUlo

PARA

OBTENÇÃO DO TÍTULO

DE

DOUTOR EM CIÊNCIAS

Programa: Estatística

Orientador: Prof. Dr. Antonio Carlos Pedroso de Lima

Durante o desenvolvimento deste trabalho o autor recebeu auxílio financeiro da CAPES/CNPq

São Paulo, junho de 2021 


\section{Uso do modelo Slash Dobrado em dados de longa duração}

Esta versão da tese contém as correções e alterações sugeridas pela Comissão Julgadora durante a defesa da versão original do trabalho, realizada em 06/10/2021. Uma cópia da versão original está disponível no

Instituto de Matemática e Estatística da Universidade de São Paulo.

Comissão Julgadora:

- Prof. Dr. Antonio Carlos Pedroso de Lima - IME-USP

- Prof ${ }^{\mathrm{a}}$. Dr ${ }^{\mathrm{a}}$. Gisela Tunes da Silva - IME-USP

- Prof. Dr. Diego Ignacio Gallardo Mateluna - Uni Atacama

- Prof. Dr. Edwin Moises Marcos Ortega - ESALQ-USP

- Prof. Dr. Eliardo Guimaraes da Costa - UFRN 


\title{
Agradecimentos
}

\author{
Gostaria de agradecer:
}

Primeiramente a Deus, nosso Pai Celestial, pois sem Ele nada seria possível e é por causa Dele que consegui superar todos os obstáculos ao longo dos meus estudos.

Aos meus pais, Luzieva e Marcos, pelos seus carinhos, conselhos, cuidados, ensinamentos e amor incondicional. Sem eles eu não teria força para seguir em frente, pois eles são o meu porto seguro. Foram eles que sempre me incentivaram a lutar pelos meus sonhos. São eles que eu sempre poderei contar em todos os momentos de minha vida. E os meus irmãos que apesar das nossas brigas, sempre me apoiaram e torceram por mim.

À minha esposa, Rosiane, que sempre esteve ao meu lado todos os dias e foi compreensiva nos momentos que não pude lhe dar a atenção que ela merece.

Tenho muito a agradecer ao meu orientador, Antonio Carlos, pois sem sua grande paciência e compreensão eu não poderia ter obtido este título que me orgulho tanto.

Não posso deixar de agradecer aos meus professores que me ensinaram tudo que eu sei, sem seus ensinamentos e conselhos eu não poderia ter chegado até aqui. Foi graças aos meus mestres que tive ao longo da minha vida de estudante que me tornei quem sou hoje.

Aos meus grandes amigos que conheci todos estes anos. Na graduação conheci pessoas incríveis que me ensinaram muito. Na residência universitária pude conviver com pessoas muito diferentes das mais diversas opiniões, elas me ajudaram muito a crescer. No IME conheci amigos que irei levar para toda a vida. Foi graças a todos os momentos felizes que tivemos que pude aproveitar ao máximo esta jornada.

Nunca poderia deixar de agradecer aos meus irmãos da Ordem DeMolay e aos meus tios Maçons. Foi graças a eles que pude melhorar as virtudes que me foram ensinadas por meus pais. São as virtudes de um DeMolay que moldaram o homem que eu sou.

Enfim, a todas as pessoas que fizeram parte de cada momento que levou à minha conclusão do mestrado. 


\section{Resumo}

CAVAlCANTE, M. R. N. Uso do modelo Slash Dobrado em dados de longa duração. 2021. 97 f. Tese (Doutorado) - Instituto de Matemática e Estatística, Universidade de São Paulo, São Paulo, 2021.

Este trabalho tem como objetivo analisar dados de longa duração. Para isto será considerada uma abordagem diferente da que é encontrada na literatura, que usualmente utiliza modelos de mistura com fração de cura. Será considerada uma distribuição paramétrica com cauda pesada, com a finalidade de acomodar dados de longo termo. Assim não há necessidade de separar os dados em curados e não curados. O que não faz muito sentido do ponto de vista filosófico, pois quando se considera o evento de interesse como sendo o óbito, não existem curados. Para avaliar o uso do modelo é feito um estudo de simulação com percentuais e tipos diferentes de censura. Covariáveis são consideradas em dois dos três parâmetros do modelo; para verificar se seus efeitos são significativos são propostos testes da razão de verossimilhanças e Wald. Utilizando os dados que motivam este trabalho é realizada uma avaliação do uso prático do modelo.

Palavras-chave: Slash dobrada, slash, normal dobrada, cauda pesada, dados de longa duração. 


\section{Abstract}

CAVAlCANTE, M. R. N. Using the Folded Normal Slash model in long-term data. 2021. 97 f. Thesis (Doctorate) - Instituto de Matemática e Estatística, Universidade de São Paulo, São Paulo, 2021.

This work aims to analyze long-term data. For this, a different approach from the one found in the literature, which usually uses mixture models with cure fraction, will be considered. A heavytailed parametric distribution will be considered, in order to accommodate long-term data. So there is no need to separate the data into healed and unhealed. Which doesn't make much sense from a philosophical point of view, because when the event of interest is considered to be death, there are no cures. To assess the use of the model, a simulation study is carried out with different percentages and types of censorship. Covariates are considered in two of the three parameters of the model; to verify whether their effects are significant, likelihood ratio and Wald tests are proposed. Using the data that motivates this work, an evaluation of the practical use of the model is carried out.

Keywords: Folded normal slash, slash, folded normal, heavy tail, long-term data. 


\section{Sumário}

$\begin{array}{ll}\text { Lista de abreviaturas } & \text { ix }\end{array}$

Lista de símbolos $\quad$ xi

Lista de Figuras $\quad$ xiii

Lista de Tabelas $\quad$ xv

1 Motivação $\quad 1$

1.1 Projeto ICESP . . . . . . . . . . . . . . . . . . . . . . . 2

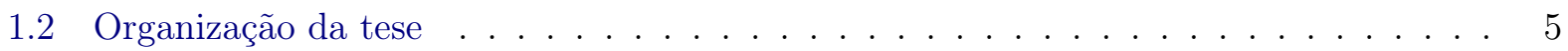

2 Distribuições Dobradas e Slash $\quad 7$

2.1 Distribuições Dobradas . . . . . . . . . . . . . . . . . . . 7

2.1.1 Família de distribuições Normal Dobrada . . . . . . . . . . . . . . . . . 7

2.2 Família de distribuições Slash . . . . . . . . . . . . . . . . . . . . . . . . . 9

2.3 Família de distribuições Slash Dobrada . . . . . . . . . . . . . . . . . . . . . . . 12

2.4 Identificabilidade no modelo Slash Dobrado . . . . . . . . . . . . . . . . . . 15

3 Distribuições Normal Dobrada e Slash Dobrada em Análise de Sobrevivência 17

3.1 Modelagem para dados censurados . . . . . . . . . . . . . . . . . . 17

3.1.1 Tempos de falha com a distribuição Normal Dobrada . . . . . . . . . . . . . 18

3.1.2 Tempos de falha com a distribuição Slash Dobrada . . . . . . . . . . . . . . . 19

3.2 Estudo de simulação . . . . . . . . . . . . . . . . . . . . . . . . 21

3.3 Teste da razão de verossimilhanças para o parâmetro $q \ldots \ldots$. . . . . . . . . . . 34

4 Heterogeneidade no modelo Slash Dobrado $\quad 39$

4.1 Inclusão de covariáveis . . . . . . . . . . . . . . . . . . . . . . . . . . 39

4.2 Teste de Wald . . . . . . . . . . . . . . . . . . . . . . . . . 42

4.3 Estudo de simulação . . . . . . . . . . . . . . . . . . . . . . . . 44

5 Aplicação aos dados do projeto ICESP $\quad 49$

5.1 Adequabilidade do modelo . . . . . . . . . . . . . . . . . . . . . . . . 49

5.2 Inclusão de covariáveis . . . . . . . . . . . . . . . . . . . . . . 50

5.3 Modelo estimado . . . . . . . . . . . . . . . . . . . . . . . . 51

5.4 Significância das covariáveis . . . . . . . . . . . . . . . . . . 52

5.5 Resíduos Cox-Snell . . . . . . . . . . . . . . . . . . . . . . . 54 
6 Considerações finais $\quad 55$

6.1 Conclusões ................................. 55

6.2 Trabalhos futuros . . . . . . . . . . . . . . . . . . . . 56

$\begin{array}{ll}\text { A Apêndice } & \mathbf{5 7}\end{array}$

A.1 Derivadas do modelo Normal Dobrado . . . . . . . . . . . . . . . . . . . . 57

A.2 Derivadas do modelo Slash Dobrado _. . . . . . . . . . . . . . . . . . . . . 61

A.3 Derivadas do modelo Slash Dobrado com covariáveis _ . . . . . . . . . . . . . . 68

$\begin{array}{ll}\text { Referências Bibliográficas } & 77\end{array}$ 


\section{Lista de abreviaturas}

$\begin{array}{ll}\text { EQM } & \text { Erro quadrático médio } \\ S L & \text { Distribuição Slash }\end{array}$ 


\title{
Lista de símbolos
}

\author{
$\phi($.$) \quad Densidade de uma distribuição normal padrão$ \\ $\Phi($.$) \quad Acumulada de uma distribuição normal padrão$ \\ $-2 \Delta($.$) Estatística do teste da razão de verossimilhanças$ \\ $I($.$) \quad Informação observada$ \\ $U($.$) \quad Função score$ \\ $L_{0}($.$) \quad Função de verossimilhança sob H_{0}$ \\ $L_{1}($.$) \quad Função de verossimilhança sob H_{1}$ \\ $l($.$) \quad Logaritmo da função de verossimilhança$
}




\section{Lista de Figuras}

1.1 Estimativas de Kaplan-Meier para os pacientes segundo o status de cirurgia . . . . . 4

1.2 Estimativas de Kaplan-Meier para os pacientes segundo o sexo . . . . . . . . . . . . 4

2.1 Função densidade de probabilidade da distribuição $\operatorname{Normal} \operatorname{Dobrada}(\mu, \sigma)$. . . . . . 8

2.2 Função densidade de probabilidade das distribuições Normal e Normal Dobrada . . . 9

2.3 Função densidade de probabilidade da distribuição $\operatorname{Slash,~} S L(0,1, q)$. . . . . . . . . 10

2.4 Função densidade de probabilidade da distribuição Slash $\operatorname{Dobrada}(\mu, \sigma, q)$. . . . . . 12

2.5 Função de sobrevivência da distribuição $\operatorname{Slash} \operatorname{Dobrada}(\mu, \sigma, q)$. . . . . . . . . . . . . 14

2.6 Função de risco da distribuição Slash $\operatorname{Dobrada}(\mu, \sigma, q)$. . . . . . . . . . . . . . . . . 15

3.1 Histogramas das estimativas dos parâmetros da distribuição Slash Dobrada(exp $(2,77)$, $4, \exp (1,10))$. Nas colunas tem-se os parâmetros, (a) $\beta_{\mu}$, (b) $\sigma$ e (c) $\beta_{q}$, com $0 \%$ de censura aleatória e 5\% tipo I em 100 réplicas. Nas linhas tem-se os tamanhos amostrais, 100, 500 e 1.000. . . . . . . . . . . . . . . . . . . . 24

3.2 Histogramas das estimativas dos parâmetros da distribuição Slash Dobrada $(\exp (2,77)$, $4, \exp (1,10))$. Nas colunas tem-se os parâmetros, (a) $\beta_{\mu}$, (b) $\sigma$ e (c) $\beta_{q}$, com $2,5 \%$ de censura aleatória e 2,5\% tipo I em 100 réplicas. Nas linhas tem-se os tamanhos amostrais, 100, 500 e 1.000. . . . . . . . . . . . . . . . . . . . 25

3.3 Histogramas das estimativas dos parâmetros da distribuição Slash Dobrada(exp $(2,77)$, $4, \exp (1,10))$. Nas colunas tem-se os parâmetros, (a) $\beta_{\mu}$, (b) $\sigma$ e (c) $\beta_{q}$, com $5 \%$ de censura aleatória e 0\% tipo I em 100 réplicas. Nas linhas tem-se os tamanhos amostrais, 100, 500 e 1.000. . . . . . . . . . . . . . . . . . . . . . 26

3.4 Histogramas das estimativas dos parâmetros da distribuição Slash Dobrada $(\exp (0,69)$, $80, \exp (-0,69))$. Nas colunas tem-se os parâmetros, (a) $\beta_{\mu}$, (b) $\sigma$ e (c) $\beta_{q}$, com $0 \%$ de censura aleatória e 25\% tipo I em 100 réplicas. Nas linhas tem-se os tamanhos amostrais, 100, 500 e 1.000. . . . . . . . . . . . . . . . . . . . . 28

3.5 Histogramas das estimativas dos parâmetros da distribuição Slash Dobrada $(\exp (0,69)$, $80, \exp (-0,69))$. Nas colunas tem-se os parâmetros, (a) $\beta_{\mu}$, (b) $\sigma$ e (c) $\beta_{q}$, com $10 \%$ de censura aleatória e 15\% tipo I em 100 réplicas. Nas linhas tem-se os tamanhos amostrais, 100, 500 e 1.000. . . . . . . . . . . . . . . . . 29

3.6 Histogramas das estimativas dos parâmetros da distribuição Slash Dobrada $(\exp (0,69)$, $80, \exp (-0,69))$. Nas colunas tem-se os parâmetros, (a) $\beta_{\mu}$, (b) $\sigma$ e (c) $\beta_{q}, \operatorname{com} 0 \%$ de censura aleatória e 50\% tipo I em 100 réplicas. Nas linhas tem-se os tamanhos amostrais, 100,500 e 1.000 . 
3.7 Histogramas das estimativas dos parâmetros da distribuição Slash Dobrada $(\exp (0,69)$, $80, \exp (-0,69)$ ). Nas colunas tem-se os parâmetros, (a) $\beta_{\mu}$, (b) $\sigma$ e (c) $\beta_{q}$, com $25 \%$ de censura aleatória e $25 \%$ tipo I em 100 réplicas. Nas linhas tem-se os tamanhos amostrais, 100, 500 e $1.000 \ldots \ldots \ldots \ldots \ldots \ldots \ldots$

3.8 Gráficos dos quantis com $0 \%$ de censura. Nas linhas $n=100$ e $n=1.000$, nas colunas quantis das distribuições qui-quadrado e Mistura de qui-quadrados . . . . . . . . . 37

3.9 Gráficos dos quantis com 30\% de censura. Nas linhas $n=100$ e $n=1.000$, nas colunas quantis das distribuições qui-quadrado e Mistura de qui-quadrados . . . . . . 37

3.10 Gráficos dos quantis com $50 \%$ de censura. Nas linhas $n=100$ e $n=1.000$, nas colunas quantis das distribuições qui-quadrado e Mistura de qui-quadrados . . . . . . 38

4.1 Histogramas das estimativas dos parâmetros $\boldsymbol{\beta}_{\mu}=(1,-3,2)$. Nas colunas tem-se os parâmetros, (a) $\beta_{\mu 0}$, (b) $\beta_{\mu 1}$ e (c) $\beta_{\mu 2}$, com $0 \%$ de censura aleatória e $25 \%$ tipo I em 100 réplicas. Nas linhas tem-se os tamanhos amostrais, 100, 500 e 1.000 . . . . . . . . 46

4.2 Histogramas das estimativas dos parâmetros $\boldsymbol{\beta}_{q}=(1,-2)$. Nas colunas tem-se os parâmetros, (a) $\beta_{q 0}$ e (b) $\beta_{q 1}$, com $0 \%$ de censura aleatória e $25 \%$ tipo I em 100 réplicas. Nas linhas tem-se os tamanhos amostrais, 100, 500 e 1.000 . . . . . . . . 46

5.1 Estimativas das curvas de sobrevivência para distribuição Slash Dobrado considerando os valores estimados. . . . . . . . . . . . . . . . . . . 53

5.2 Resíduos Cox-Snell para o modelo Slash Dobrado e alguns modelos de mistura. . . . 54 


\section{Lista de Tabelas}

1.1 Características demográficas para os pacientes do projeto ICESP $\ldots \ldots$. . . . . . 2

1.2 Características clínicas e de tratamento para os pacientes do projeto ICESP . . . . . 3

1.3 Descritiva dos tempos $($ em dias $) \ldots \ldots \ldots \ldots \ldots \ldots$

3.1 Número de iterações até a convergência do algoritmo. . . . . . . . . . . . . . . . . . . 22

3.2 Estimativas para os parâmetros da distribuição Slash Dobrada $(16,4,3)$ com $5 \%$ de censura e 100 réplicas de tamanhos 100,500 e $1.000 \ldots \ldots$. . . . . . . . . . . 23

3.3 Estimativas para os parâmetros da distribuição Slash Dobrada $(2 ; 80 ; 0,5)$ com $25 \%$ de censura, em 100 réplicas de tamanho igual a 100, 500 e 1.000 . . . . . . . . . . 27

3.4 Estimativas para os parâmetros da distribuição Slash Dobrada $(2 ; 80 ; 0,5)$ com $50 \%$ de censura, em 100 réplicas de tamanho igual a 100, 500 e 1.000 . . . . . . . . . . 30

3.5 Erro tipo I cometido em 1.000 réplicas. . . . . . . . . . . . . . . . . . . . 36

4.1 Estimativas para os parâmetros da distribuição $\boldsymbol{\beta}_{\mu}=(1,-3,2)$ com $25 \%$ de censura, em 100 réplicas de tamanho igual a 100,500 e $1.000 \ldots \ldots$. . . . . . . . . . 45

4.2 Estimativas para os parâmetros da distribuição $\boldsymbol{\beta}_{q}=(1,-2)$ com $25 \%$ de censura, em 100 réplicas de tamanho igual a 100,500 e $1.000 \ldots \ldots$. . . . . . . . . . 45

5.1 Estimativas de máxima verossimilhança para os parâmetros do modelo Slash Dobrado aplicado ao projeto ICESP. . . . . . . . . . . . . . . . . . 51

5.2 Valor p do teste de Wald para as covariáveis do modelo Slash Dobrado. . . . . . . . . 53

5.3 AIC dos modelos. . . . . . . . . . . . . . . . . . . . . . . 54 


\section{Capítulo 1}

\section{Motivação}

Em estudos de sobrevivência, o principal objetivo é estudar o tempo até a observação de um ou mais eventos de interesse. Alguns exemplos referem-se ao tempo até o reaparecimento de um certo tipo de câncer, cura de uma doença após um tratamento ou morte de pacientes terminais. O desenvolvimento inicial tem como pressuposto que, se uma unidade amostral pudesse ser acompanhada por um período suficientemente longo, o evento de interesse seria efetivamente observado. Mais recentemente, modelos que assumem a possibilidade de o evento não ser observado para parte da população têm recebido bastante atenção na literatura, como por exemplo Pitkäniemi et al. [2007] e Locatelli et al. [2007]. Tais modelos têm sua gênese nos trabalhos de Boag [1949] e Berkson \& Gage [1952] e são tratados em detalhes em Maller \& Zhou [1996], recebendo a denominação de modelos de mistura.

Alternativamente aos modelos com misturas de distribuições, Yakolev \& Tsodikov [1996] propuseram modelos com estrutura de taxa de falhas competitivas, abordagem posteriormente estendida por Chen et al. [1999], dando origem aos modelos de tempo de promoção. Rodrigues et al. [2009] propuseram uma classe de modelos que unifica os modelos de mistura e de tempo de promoção.

Os modelos de mistura e/ou tempos de promoção têm sido utilizados para a modelagem de dados em que parte dos indivíduos, denominados curados, não são suscetíveis ao evento de interesse, mesmo que fossem acompanhados por um período muito longo. Esses modelos também têm sido utilizados em situações em que o evento leva um tempo muito maior do que o esperado, sendo que todos os indivíduos são passíveis de apresentar o evento de interesse. Para a segunda situação as abordagens que assumem a presença de curados contraria a filosofia utilizada para a definição de tais modelos, o que motiva a busca por procedimentos alternativos que viabilizem com maior realismo a presença de indivíduos que, apesar de em um tempo maior do que o esperado, irão eventualmente apresentar 
o evento de interesse.

Este trabalho é motivado por um conjunto de dados reais envolvendo pacientes oncológicos em estado terminal, para os quais espera-se uma baixa expectativa de vida. Entretanto, uma parcela desses indivíduos apresenta um tempo de sobrevivência incomumente alto, sendo muitas vezes censurados devido ao término do período planejado para a realização do estudo. Denominaremos tais tempos como dados de longa duração ou de longo termo.

Um interesse natural é verificar o que influencia a ocorrência de dados de longo termo. Para isto é interessante o uso de distribuições com cauda pesada, que possibilitam a atribuição de maiores probabilidades para valores com maior distância em relação à média. Dentro desse contexto, o principal foco deste estudo será avaliar o uso da distribuição Slash Dobrada, na literatura é comum o uso do termo Folded Normal Slash (ver Gui et al. [2013]), que possui um parâmetro específico para controle do peso da cauda. Esse parâmetro pode ser utilizado para verificar características que aumentam a chance de ocorrência de dados de longo termo. A distribuição Slash Dobrada é bem flexível, podendo modelar bem dados com grande assimetria e casos em que o tempo observado é maior que o tempo esperado.

Na próxima seção é descrito o conjunto de dados que motiva este trabalho.

\subsection{Projeto ICESP}

Normilio-Silva et al. [2016] consideraram um estudo envolvendo 792 pacientes oncológicos em estado terminal. A coleta dos dados ocorreu entre março de 2010 a agosto de 2011, sendo concluído o acompanhamento de todos os pacientes em agosto de 2013.

Para este trabalho será considerado o conjunto de dados contendo 803 pacientes, sendo 191 que deram entrada na UTI do Hospital do Câncer de Barretos - SP e 612 na UTI do Instituto do Câncer do Estado de São Paulo (ICESP). Em ambos os centros não existe diferença entre os protocolos de atendimento aos pacientes.

Tabela 1.1: Características demográficas para os pacientes do projeto ICESP

\begin{tabular}{cccccc}
\hline \hline \multirow{2}{*}{ Sexo } & Feminino & Masculino & & & \\
& $341(42 \%)$ & $462(58 \%)$ & & & \\
\hline Idade & Média & Erro padrão & $1^{\circ}$ quartil & Mediana & $3^{\circ}$ quartil \\
(anos) & 61 & 14 & 53 & 63 & 71 \\
\hline Peso & Média & Erro padrão & $1^{\circ}$ quartil & Mediana & $3^{\circ}$ quartil \\
(quilos) & 65 & 15 & 54 & 63 & 74 \\
\hline \hline
\end{tabular}


Tabela 1.2: Características clínicas e de tratamento para os pacientes do projeto ICESP

\begin{tabular}{|c|c|c|c|c|}
\hline & \multirow[b]{2}{*}{ Status do câncer } & \multicolumn{2}{|r|}{ Ativo } & \multirow{2}{*}{$\begin{array}{c}\text { Controlado } \\
\text { remissão } \\
49(6 \%)\end{array}$} \\
\hline & & $\begin{array}{l}\text { recente } \\
408(51 \%)\end{array}$ & $\begin{array}{c}\text { recaída } \\
344(43 \%)\end{array}$ & \\
\hline & Status de cirurgia & $\begin{array}{l}\text { Agendada } \\
410(51 \%)\end{array}$ & $\begin{array}{c}\text { Urgência ou não cirúrgico } \\
392(49 \%)\end{array}$ & \\
\hline \multirow{3}{*}{ Tratamentos } & Cirurgia & $\begin{array}{c}\text { Não } \\
317(40 \%)\end{array}$ & $\begin{array}{l}\text { Curativa } \\
428(53 \%)\end{array}$ & $\begin{array}{l}\text { Paliativa } \\
57(7 \%)\end{array}$ \\
\hline & Radioterapia & $\begin{array}{c}\text { Não } \\
627(78 \%)\end{array}$ & $\begin{array}{l}\text { Curativa } \\
148(19 \%)\end{array}$ & $\begin{array}{l}\text { Paliativa } \\
25(3 \%)\end{array}$ \\
\hline & Quimioterapia & $\begin{array}{c}\text { Não } \\
497(62 \%)\end{array}$ & $\begin{array}{l}\text { Curativa } \\
216(27 \%)\end{array}$ & $\begin{array}{l}\text { Paliativa } \\
88(11 \%)\end{array}$ \\
\hline & Dias de UTI & $\begin{array}{l}\text { Média } \\
5,0\end{array}$ & $\begin{array}{c}\text { Erro padrão } \\
5,6\end{array}$ & \\
\hline
\end{tabular}

Tabela 1.3: Descritiva dos tempos (em dias)

\begin{tabular}{cccccc}
\hline \hline Tempo até & Média & Desvio padrão & $1^{\circ}$ quartil & Mediana & $3^{\circ}$ quartil \\
o óbito & 135 & 178 & 13 & 46 & 194 \\
\hline Tempo até & Média & Desvio padrão & $1^{\circ}$ quartil & Mediana & $3^{\circ}$ quartil \\
a censura & 690 & 158 & 720 & 724 & 733 \\
\hline Censura & $35 \%$ & & & & \\
\hline \hline
\end{tabular}

Uma covariável de interesse é a que indica se o paciente passou por cirurgia agendada ou não agendada. Na Figura 1.1 verifica-se a diferença entre os tempos de sobrevida dos dois grupos; notase que o grupo composto por pacientes com cirurgia agendada tem-se $50 \%$ de censura, ao passo que para o grupo com cirurgia não agendada essa porcentagem é de $20 \%$. Verifica-se que as funções de sobrevivência estimadas não caem para zero na velocidade esperada. 


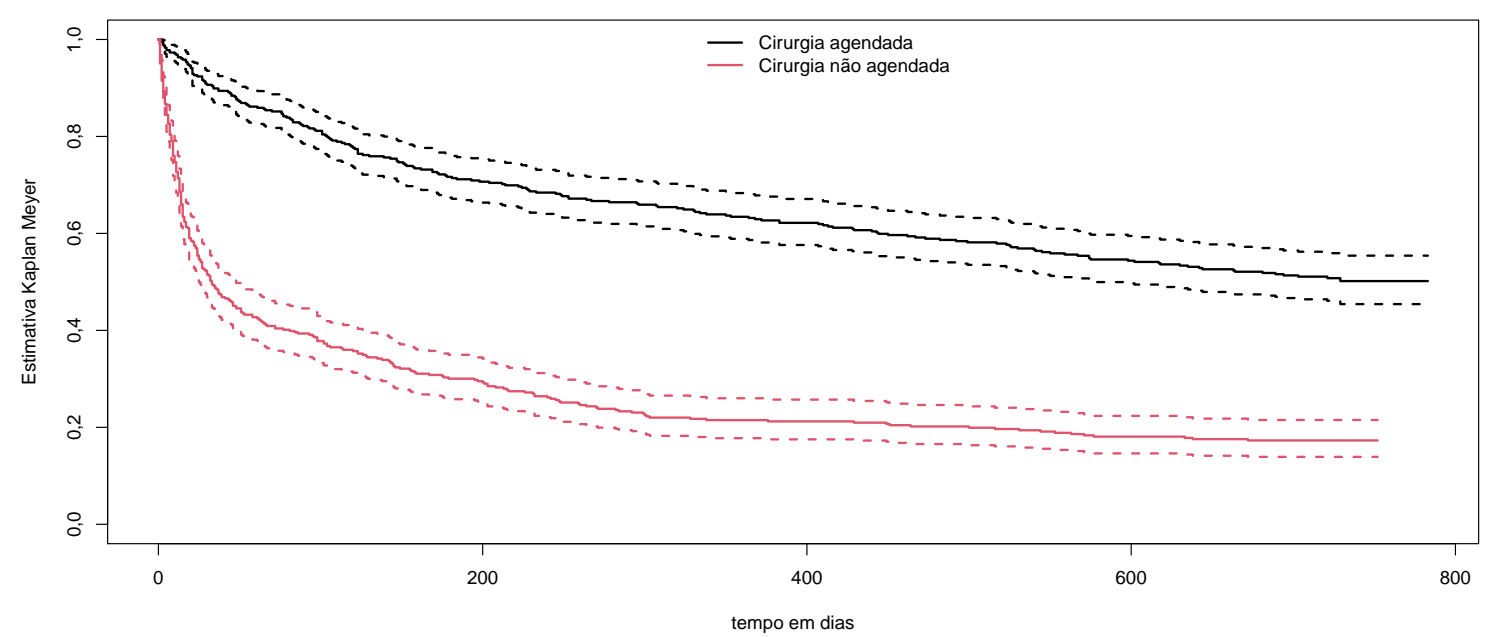

Figura 1.1: Estimativas de Kaplan-Meier para os pacientes segundo o status de cirurgia

Outras covariáveis que podem ser consideradas são o sexo e a idade dos pacientes. Espera-se que a sobrevida decaia conforme a idade aumenta. Assim como é comum existir diferença na sobrevida de pacientes de sexos diferentes.

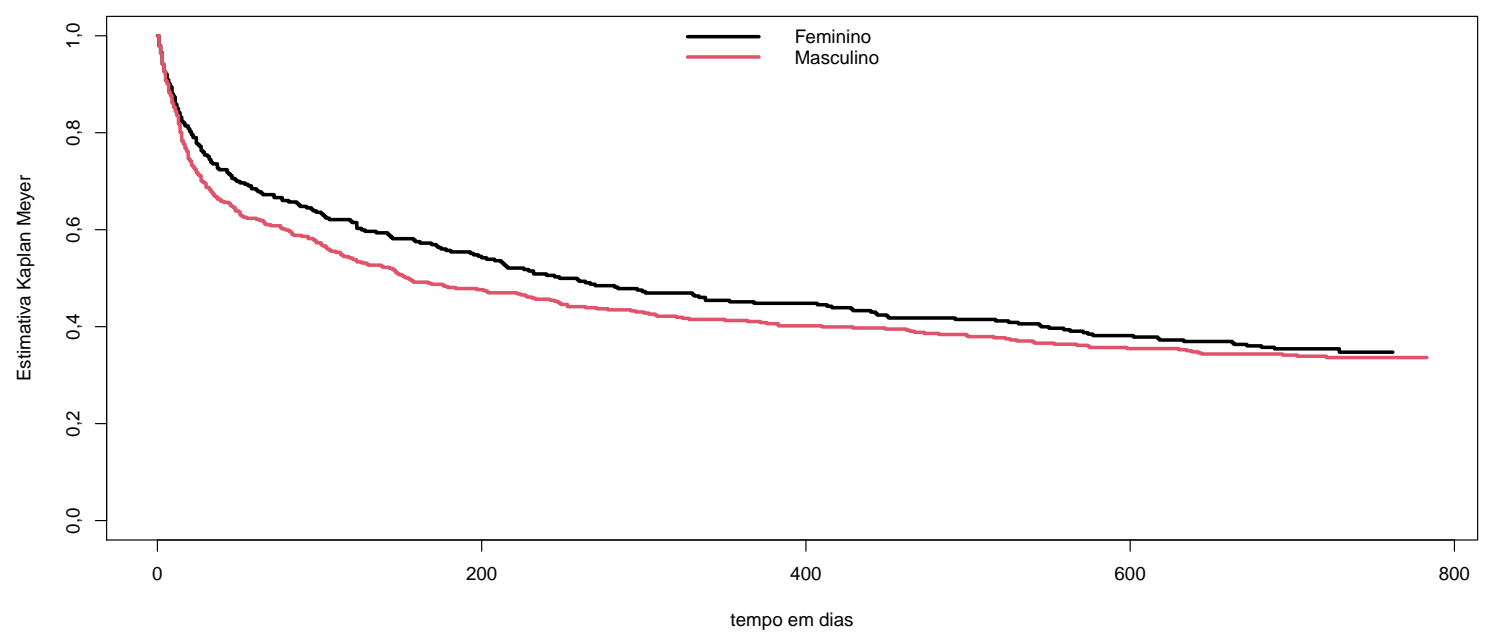

Figura 1.2: Estimativas de Kaplan-Meier para os pacientes segundo o sexo

Pela Figura 1.1 verifica-se que as curvas estimadas de sobrevivência são consideravelmente diferentes, mostrando a heterogeneidade presente nos dados. Para estudar essa heterogeneidade serão incluídas covariáveis nos parâmetros de posição e que controla o peso da cauda. Pela Figura 1.2 nota-se que não existe uma diferença significativa entre as curvas estimadas de sobrevivência. No entanto, a curva de sobrevivência estimada para as mulheres está acima da dos homens. Assim é interessante verificar se o sexo influencia na sobrevida dos pacientes. 


\subsection{Organização da tese}

O presente trabalho está dividido em seis capítulos. No segundo capítulo, são apresentadas as definições das distribuições Dobradas e Slash e algumas de suas propriedades, bem como as funções densidade de probabilidade, sobrevivência e risco. A identificabilidade do modelo é discutida.

No terceiro capítulo, as distribuições Normal Dobrada e Slash Dobrada são utilizadas sob o enfoque de análise de sobrevivência. São apresentados também os estimadores de máxima verossimilhança para os parâmetros do modelo, bem como discutido o teste da razão de verossimilhanças. Nesse capítulo também é apresentado um estudo de simulação.

O quarto capítulo considera a inclusão de covariáveis em dois parâmetros do modelo Slash Dobrado. Neste contexto é discutido o uso do teste de Wald para verificar se as covariáveis são estatisticamente significativas. O comportamento dos estimadores de máxima verossimilhança é avaliado através de simulações.

No quinto capítulo é apresentada uma aplicação do modelo Slash Dobrado aos dados ICESP que motivaram a tese.

Por fim, no sexto capítulo, são apresentadas conclusões dos resultados obtidos e perspectivas de trabalhos futuros. 


\section{Capítulo 2}

\section{Distribuições Dobradas e Slash}

Neste capítulo são apresentadas duas famílias de distribuições bastante úteis, distribuições $D o-$ bradas e distribuições Slash. Essas distribuições são adequadas para modelar dados com forte assimetria. No caso da distribuição Slash ainda é possível controlar o peso da cauda das distribuições através de um parâmetro próprio.

\subsection{Distribuições Dobradas}

As distribuições Dobradas (em inglês folded distributions) são obtidas ao aplicar o módulo em alguma variável aleatória que assuma valores nos reais, como as distribuições Normal, t-student, Laplace, dentre outras. Nestes casos, ao aplicar o módulo obtém-se as distribuições Folded Normal (Leone et al. [1961]), Folded $t$ (FT) (Psarakis \& Panaretoes [1990]) e Folded Laplace (FL) (Liu \& Kozubowski [2015]). Neste trabalho será utilizada a distribuição Folded Normal, que será denominada Normal Dobrada, esta será mostrada em maior detalhe. Para as outras distribuições, sugere-se ao leitor consultar as referências citadas.

\subsubsection{Família de distribuições Normal Dobrada}

A distribuição Normal Dobrada é uma alternativa à distribuição Normal nos casos em que é necessário modelar variáveis não negativas.

Considere a distribuição Normal, $W \sim \operatorname{Normal}\left(\mu, \sigma^{2}\right)$, com parâmetros de posição $\mu \in \mathbb{R}$ e de escala $\sigma \in \mathbb{R}_{+}$, cuja função densidade de probabilidade (f.d.p.) é dada por

$$
f_{W}(w)=\frac{1}{\sqrt{2 \pi \sigma^{2}}} e^{-\frac{(w-\mu)^{2}}{2 \sigma^{2}}} I_{\mathbb{R}}(w)
$$


Fazendo $X=|W|$ obtém-se a distribuição Normal Dobrada $(\mu, \sigma)$, cuja f.d.p. é dada por

$$
f_{X}(x)=\frac{1}{\sqrt{2 \pi \sigma^{2}}}\left[e^{-\frac{(x-\mu)^{2}}{2 \sigma^{2}}}+e^{-\frac{(x+\mu)^{2}}{2 \sigma^{2}}}\right] I_{\mathbb{R}_{+}}(x) .
$$

A função de sobrevivência da distribuição Normal Dobrada é dada por

$$
S_{X}(x)=\int_{x}^{\infty} \frac{1}{\sqrt{2 \pi \sigma^{2}}}\left[e^{-\frac{(y-\mu)^{2}}{2 \sigma^{2}}}+e^{-\frac{(y+\mu)^{2}}{2 \sigma^{2}}}\right] \mathrm{d} y
$$

Na Figura 2.1 tem-se a f.d.p da Normal Dobrada para alguns valores de $\mu$ e $\sigma$. Sem perda de generalidade pode-se considerar valores não negativos para $\mu$. Conforme a diferença $\mu-\sigma$ fica mais positiva, a curva da f.d.p. se torna mais simétrica. Já quando essa diferença fica mais próxima de zero, a f.d.p. obtém uma assimetria positiva. Devida a essa diferença indicar o quão assimétrico pode ser um conjunto de dados, pode-se incluir covariáveis nos parâmetros para assim separar grupos de indivíduos com assimetrias diferentes.

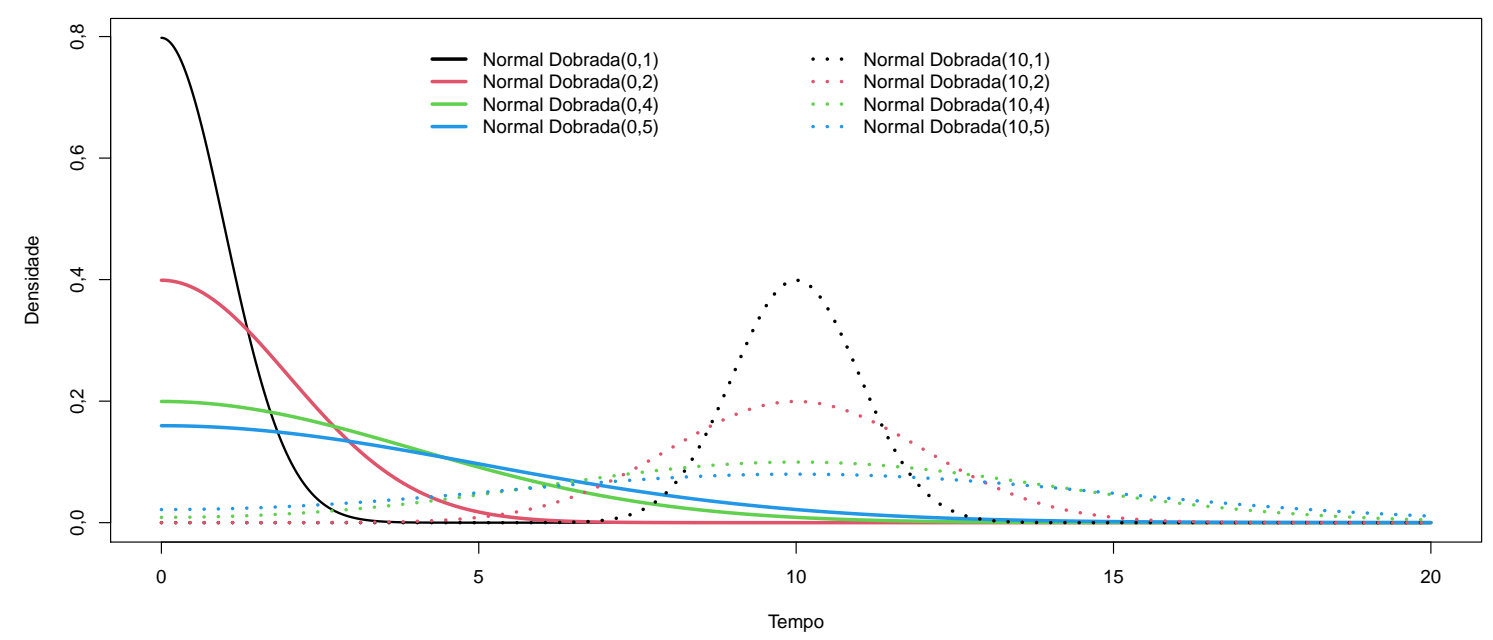

Figura 2.1: Função densidade de probabilidade da distribuição Normal Dobrada $(\mu, \sigma)$

Leone et al. [1961] consideraram a reparametrização $\theta=\mu / \sigma \in \mathbb{R}$, obtendo assim a distribuição Normal Dobrada $(\theta, \sigma)$, cuja f.d.p. é dada por

$$
f_{X}(x)=\frac{\sqrt{2}}{\sigma \sqrt{\pi}} e^{-\frac{\theta^{2}}{2}} e^{-\frac{x^{2}}{2 \sigma^{2}}} \cosh \left(\frac{\theta x}{\sigma}\right) I_{\mathbb{R}_{+}}(x),
$$

em que $\cosh (\theta)=\frac{e^{\theta}+e^{-\theta}}{2}$ é o cosseno hiperbólico.

Neste trabalho será considerada a parametrização dada em (2.1). Esta parametrização foi escolhida para facilitar o algoritmo computacional e a interpretação. Na parametrização em (2.3) 
existe uma relação entre $\theta$ e $\sigma$, o que não ocorre em (2.1). A ortogonalidade entre os parâmetros é importante para facilitar a interpretação quando inclui-se covariáveis no modelo.

Tsagris et al. [2014] falaram da relação entre os parâmetros $\mu$ e $\sigma$. Quando $\mu$ é menor que $\sigma$, a distribuição Normal Dobrada tem uma assimetria maior. Isso ocorre pois quanto menor for o quociente $\mu / \sigma$ maior a probabilidade da distribuição Normal ter valores negativos, de tal forma que o módulo resulta numa assimetria. Em contra partida, quanto maior for o quociente $\mu / \sigma$ mais simétrica é a distribuição Normal Dobrada. Na Figura 2.2 verifica-se essa relação entre os parâmetros.
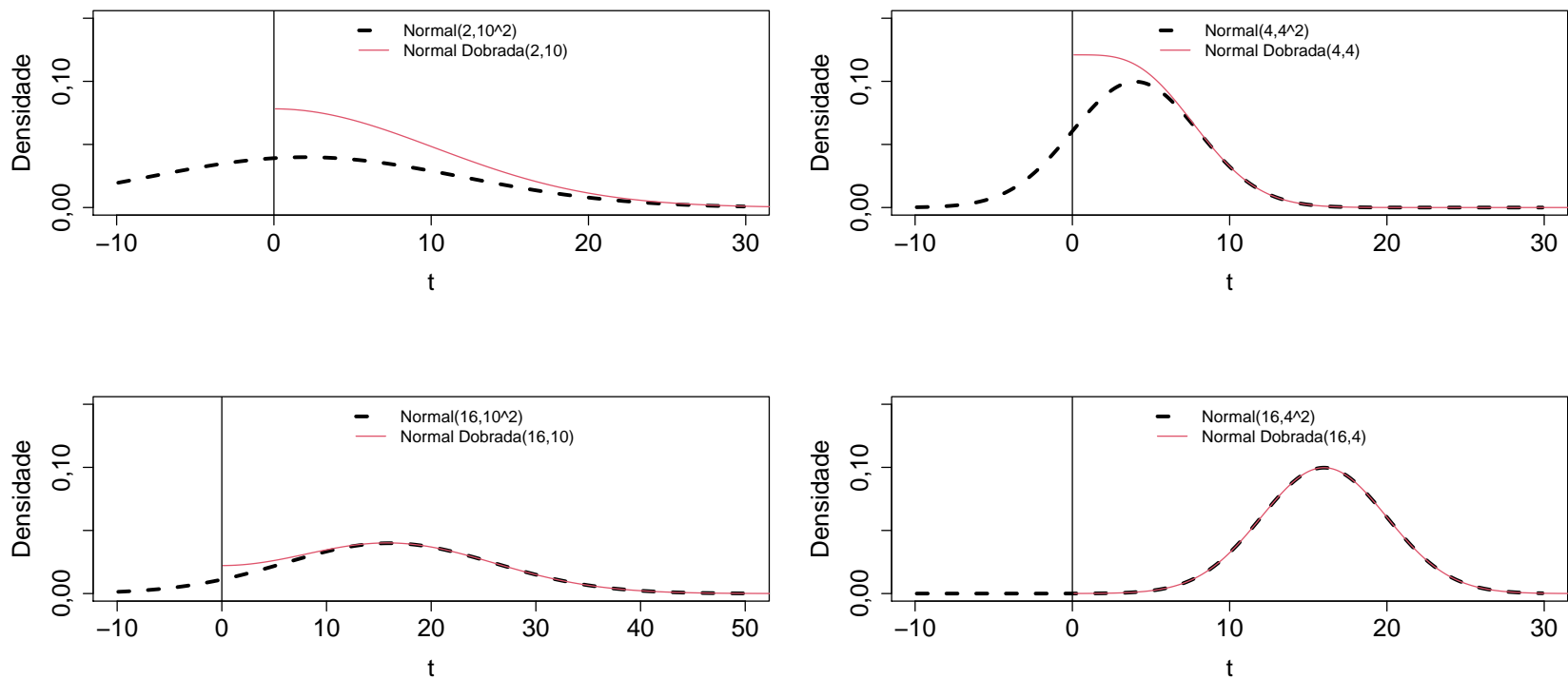

Figura 2.2: Função densidade de probabilidade das distribuições Normal e Normal Dobrada

\subsection{Família de distribuições Slash}

Wang \& Genton [2006] apresentaram como definição para a distribuição Slash o seguinte quociente

$$
S=\frac{Z}{U^{1 / q}} \sim S L(0,1, q), \quad q>0
$$

com $Z \sim \operatorname{Normal}(0,1)$ independente de $U \sim \operatorname{Uniforme}(0,1)$.

Considerando a transformação biunívoca $M=U^{1 / q}$, obtém-se

$$
F_{M}(m)=P(M \leq m)=P\left(U^{1 / q} \leq m\right)=P\left(U \leq m^{q}\right)=F_{U}\left(m^{q}\right) .
$$

Como $U \sim$ Uniforme $(0,1)$, então $F_{U}(u)=u I_{(0,1)}(u)+I_{[1, \infty)}(u)$. Assim, 


$$
F_{M}(m)=m^{q} I_{(0,1)}(m)+I_{[1, \infty)}(m) \Longrightarrow f_{M}(m)=q m^{q-1} I_{(0,1)}(m) .
$$

Desta forma, $U^{1 / q} \sim \operatorname{Beta}(q, 1)$.

Logo, pode-se utilizar a seguinte definição para a distribuição Slash. Define-se uma variável aleatória, $S$, com distribuição Slash quando esta é dada por

$$
S=\frac{Z}{U} \sim S L(0,1, q), \quad q>0
$$

sendo $Z \sim \operatorname{Normal}(0,1)$ independente de $U \sim \operatorname{Beta}(q, 1)$, em que

$$
f_{Z}(z)=\frac{e^{-z^{2} / 2}}{\sqrt{2 \pi}} I_{\mathbb{R}}(z) \text { e } f_{U}(u)=q u^{q-1} I_{(0,1)}(u) .
$$

Na Figura 2.3 tem-se a f.d.p da $S L$ para alguns valores de $q$.

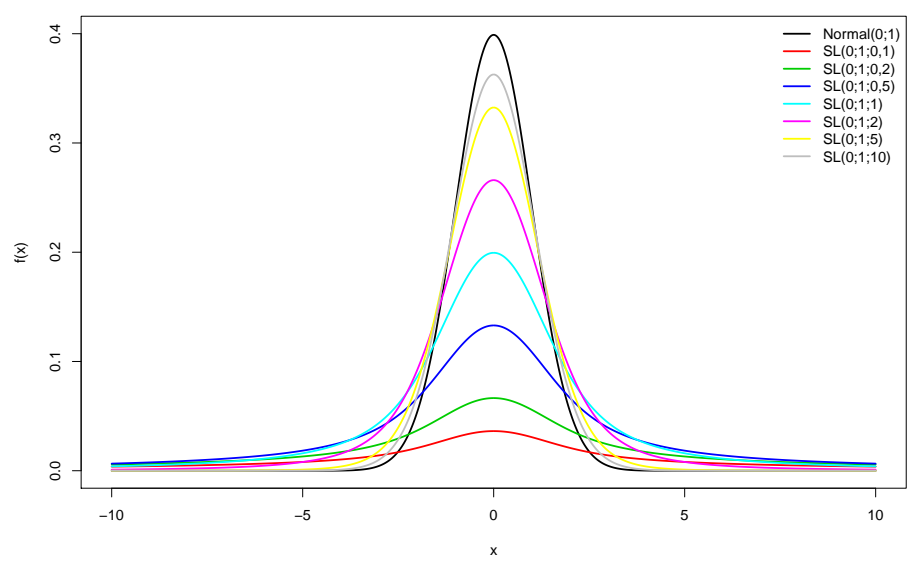

Figura 2.3: Função densidade de probabilidade da distribuição Slash, $S L(0,1, q)$

Se $q=1$, obtém-se a distribuição Slash padrão, ou na forma canônica, que possui função densidade de probabilidade mais simplificada.

$$
f_{S}(s)=\left\{\begin{array}{ccc}
\frac{\phi(0)-\phi(s)}{s^{2}}, & \text { se } & s \neq 0 \\
\frac{\phi(0)}{2}, & \text { se } & s=0
\end{array}\right.
$$

sendo $\phi(y)=\frac{1}{\sqrt{2 \pi}} e^{-\frac{y^{2}}{2}}$ a f.d.p. da distribuição Normal padrão.

Pode-se verificar também, que a distribuição Slash só possui esperança para $q>1$, sendo $E[S]=0$, e só possui variância para $q>2$, sendo $\operatorname{Var}[S]=\frac{q}{q-2}$. 
O excesso de curtose, $E C$, é dado por

$E C=\frac{E\left[(S-E[S])^{4}\right]}{E\left[(S-E[S])^{2}\right]^{2}}-3=\frac{E\left[S^{4}\right]}{E\left[S^{2}\right]^{2}}-3=3 \frac{q}{q-4}\left(\frac{q-2}{q}\right)^{2}-3=3\left(\frac{q^{2}-4 q+4}{q^{2}-4 q}-1\right)=\frac{12}{q(q-4)}$.

Nota-se que só é possível calcular a curtose para $q>4$, e que $E C>0$. Assim a distribuição Slash é leptocúrtica, ou seja, possui cauda mais pesada do que a distribuição normal. Quando q aumenta o excesso de curtose tende a zero.

Para obter uma distribuição Slash com parâmetros de posição e escala, quando há interesse, basta usar a propriedade de linearidade (ver Wang \& Genton [2006]). Ou seja, se o interesse é obter uma distribuição com parâmetros de posição e escala, respectivamente, $\mu$ e $\sigma$ é só utilizar a distribuição Slash como definida, e em seguida, fazer uma transformação linear. O modelo está, portanto, na classe dos modelos de localização-escala. Assim se

$$
S \sim S L(0,1, q) \text {, então } X=\mu+\sigma S \sim S L(\mu, \sigma, q) .
$$

Dependendo dos valores dos parâmetros a distribuição pode ter uma dispersão muito grande e uma cauda mais pesada, comparativamente à distribuição Normal padrão: quando $q$ cresce a distribuição $S L$ se aproxima da distribuição Normal padrão. Para maiores detalhes sobre a distribuição Slash (ver Cavalcante [2015]).

$\mathrm{Na}$ literatura existem muitos trabalhos sobre a distribuição Slash e variações utilizando o mesmo raciocínio mencionado anteriormente de tomar um quociente com uma distribuição Beta. Olmos et al. [2012] trabalharam com a distribuição Slashed Half-normal, que é um caso particular da distribuição Slash com $\mu=0$. Gómez \& De Castro [2019] trabalharam com este modelo em análise de regressão. Pode-se ainda considerar outras distribuições no lugar da distribuição Normal e assim obter variações da distribuição Slash.

Na presença de dados de longa duração, torna-se conveniente juntar as duas classes de distribuição, Dobradas e Slash. Como exemplos podem ser citadas as distribuições Slash Dobrada (Gui et al. [2013]) e Folded Laplace Slash (FLS) (Nojoumizadeh \& Saberi [2019]). 


\subsection{Família de distribuições Slash Dobrada}

Neste trabalho esta família de distribuições será denominada Slash Dobrada (em inglês Folded Normal Slash) e pode ser obtida da seguinte forma: sejam as variáveis aleatórias independentes $X \sim$ Normal Dobrada $(\mu, \sigma)$ e $U \sim \operatorname{Beta}(q, 1)$. Então, a variável aleatória

$$
T=X / U
$$

tem distribuição Slash Dobrada, cuja notação a ser utilizada é Slash Dobrada $(\mu, \sigma, q)$, com $\mu \in \mathbb{R}$, $\sigma \in \mathbb{R}_{+}$e $q \in \mathbb{R}_{+}$. A f.d.p. é dada por

$$
f_{T}(t)=\int_{0}^{1} \frac{q u^{q}}{\sigma \sqrt{2 \pi}}\left[e^{-\frac{(u t-\mu)^{2}}{2 \sigma^{2}}}+e^{-\frac{(u t+\mu)^{2}}{2 \sigma^{2}}}\right] \mathrm{d} u=\int_{0}^{1} \frac{q u^{q}}{\sigma}\left[\phi\left(\frac{u t-\mu}{\sigma}\right)+\phi\left(\frac{u t+\mu}{\sigma}\right)\right] \mathrm{d} u I_{\mathbb{R}_{+}}(t) .
$$

Nas Figuras 2.4, 2.5 e 2.6 em cada um dos quatro gráficos o valor de $q$ é o mesmo, dessa forma, a variação entre as f.d.p.s em um mesmo gráfico deve-se a mudanças nos outros parâmetros. Dentro de cada gráfico, as linhas com o mesmo padrão, contínuas ou tracejadas, o único parâmetro que se altera é o $\sigma$. Já nas linhas da mesma cor o único parâmetro que se altera é o $\mu$.
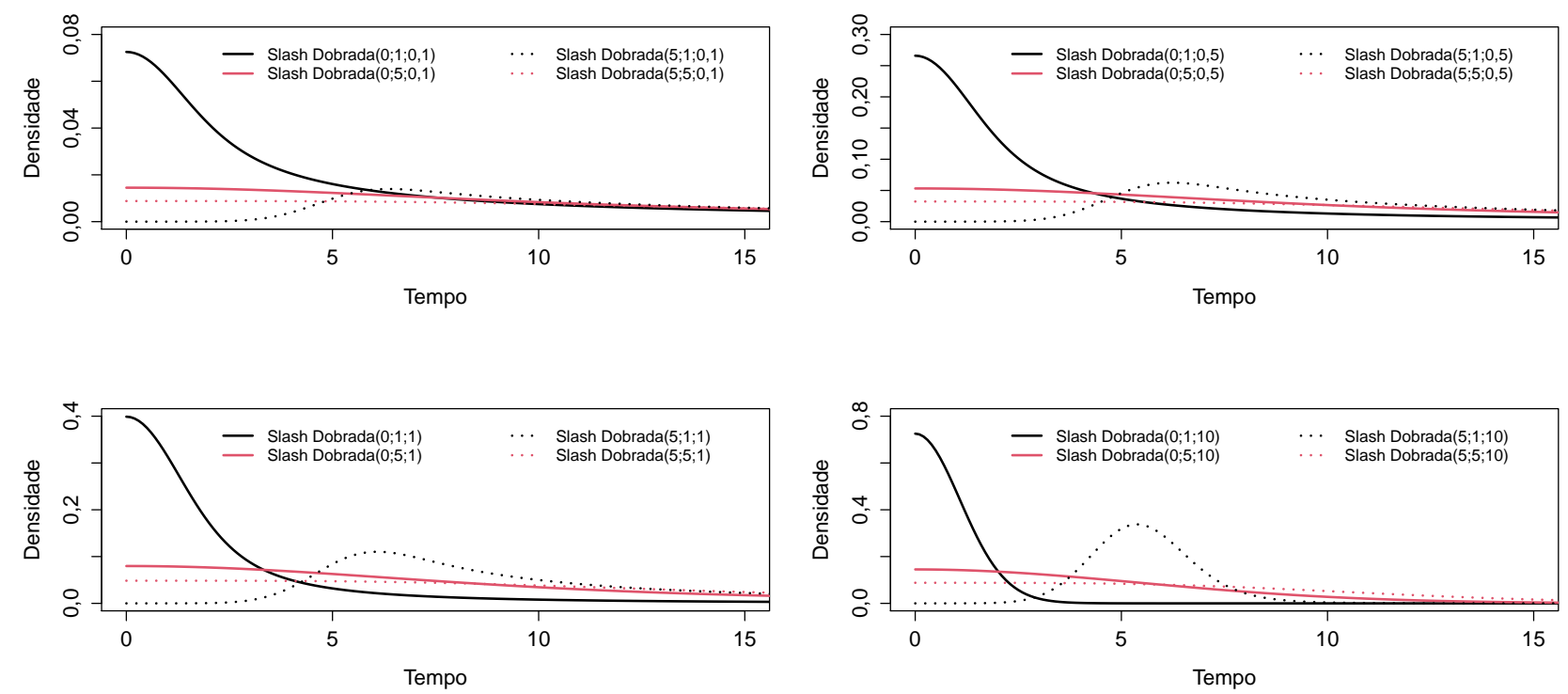

Figura 2.4: Função densidade de probabilidade da distribuição Slash Dobrada $(\mu, \sigma, q)$

A Figura 2.4 ilustra o comportamento da f.d.p. da distribuição Slash Dobrada. Nota-se que dependendo dos valores dos parâmetros, a distribuição possui cauda mais pesada, ou seja, a curva da função densidade decresce mais lentamente. Variações em qualquer parâmetro influenciam na 
concentração dos dados, sendo que para valores menores de $q$, deixando-se fixados os valores dos outros parâmetros, a distribuição possui cauda mais pesada. Tem-se, ainda, que quando o valor de $q$ aumenta a distribuição Slash Dobrada tende à distribuição Normal Dobrada. Para facilitar na visualização dos gráficos foram utilizadas escalas diferentes no eixo $y$.

Assim nota-se que modificando $\mu$ e $\sigma$, a localização e a concentração dos dados muda. Também, o parâmetro $q$ causa alterações mais significativas na curva da distribuição.

A distribuição Slash Dobrada $(\mu, \sigma, q)$ possui média e variância dadas por

$$
\begin{aligned}
E[T] & =\frac{q}{q-1}\left[2 \sigma \phi\left(\frac{\mu}{\sigma}\right)+2 \mu \Phi\left(\frac{\mu}{\sigma}\right)-\mu\right], \quad q>1 \\
\operatorname{Var}(T) & =\frac{q\left(\mu^{2}+\sigma^{2}\right)}{q-2}-\frac{q^{2}}{(q-1)^{2}}\left[2 \sigma \phi\left(\frac{\mu}{\sigma}\right)+2 \mu \Phi\left(\frac{\mu}{\sigma}\right)-\mu\right]^{2}, \quad q>2,
\end{aligned}
$$

sendo $\Phi(k)=\int_{-\infty}^{k} \frac{1}{\sqrt{2 \pi}} e^{-\frac{y^{2}}{2}} \mathrm{~d} y$ a função distribuição acumulada da distribuição Normal padrão.

Para $q<1$ a média da distribuição Slash Dobrada não existe e para $q<2$ a variância não existe.

A função de sobrevivência da distribuição Slash Dobrada é dada por

$$
S_{T}(t)=\int_{0}^{1} \frac{q u^{q}}{\sigma \sqrt{2 \pi}} \int_{t}^{\infty}\left[e^{-\frac{(u y-\mu)^{2}}{2 \sigma^{2}}}+e^{-\frac{(u y+\mu)^{2}}{2 \sigma^{2}}}\right] \mathrm{d} y \mathrm{~d} u=q \int_{0}^{1} u^{q-1}\left[S\left(\frac{u t-\mu}{\sigma}\right)+S\left(\frac{u t+\mu}{\sigma}\right)\right] \mathrm{d} u
$$

em que $S(k)=1-\Phi(k)$.

Escrevendo $S_{T}(t)$ em função da função de sobrevivência da distribuição Normal obtém-se ganhos computacionais.

A Figura 2.5 ilustra a função de sobrevivência da distribuição Slash Dobrada. Nota-se claramente que quanto menor for o valor de $q$, mais pesada é a cauda da distribuição, acomodando assim tempos de sobrevivência maiores. A curva da função de sobrevivência no primeiro gráfico decresce muito lentamente, devido ao valor pequeno de $q$; já no quarto gráfico a curva da função de sobrevivência decresce bem rapidamente, sendo o valor de $q$ maior. 

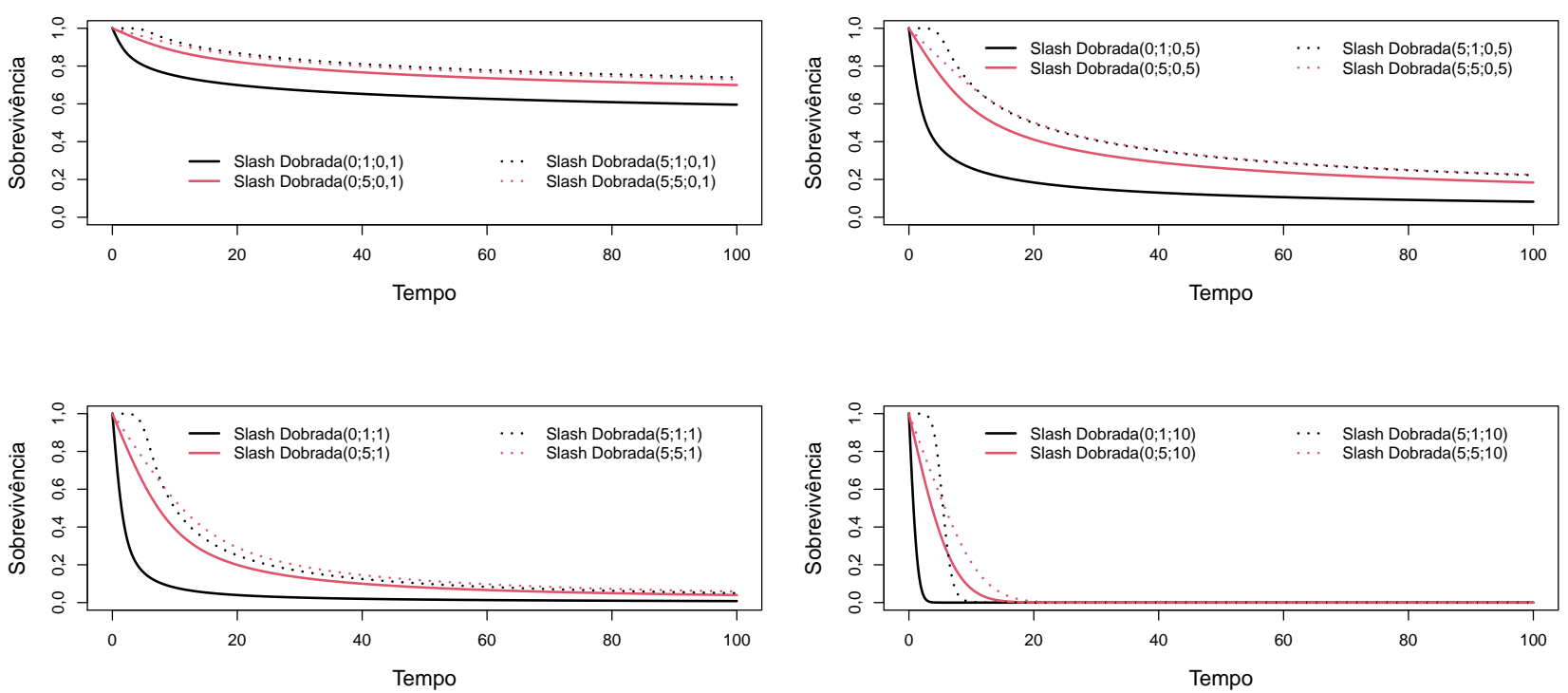

Figura 2.5: Função de sobrevivência da distribuição Slash Dobrada $(\mu, \sigma, q)$

A função de risco ou de taxa de falha da distribuição Slash Dobrada é dada por

$$
\lambda_{T}(t)=\frac{f_{T}(t)}{S_{T}(t)}=\frac{\int_{0}^{1} \frac{u^{q}}{\sigma}\left[\phi\left(\frac{u t-\mu}{\sigma}\right)+\phi\left(\frac{u t+\mu}{\sigma}\right)\right] \mathrm{d} u}{\int_{0}^{1} u^{q-1}\left[S\left(\frac{u t-\mu}{\sigma}\right)+S\left(\frac{u t+\mu}{\sigma}\right)\right] \mathrm{d} u} .
$$

Na Figura 2.6 nota-se que quanto menor for o valor do parâmetro $q$ menor a área sob a função de risco. Para valores pequenos de $q$ a probabilidade de sobrevivência em tempos mais elevados aumenta. Em contrapartida, conforme o valor de $q$ aumenta o risco vai se concentrando mais no início do estudo. Essa característica é interessante quando existem grupos de indivíduos que demoram mais para apresentar o evento de interesse e grupos que apresentam o evento mais no início do estudo. Nota-se ainda que essa distribuição pode ser adequada para modelar situações em que o risco aumenta no início do tempo, decrescendo após certo instante, como em casos em que pacientes passam por uma cirurgia ou tratamento de emergência. Nesses casos, se o evento de interesse for o óbito, seu risco aumenta antes dessas intervenções, atingindo o máximo no momento da intervenção, e depois decresce conforme o paciente vai se recuperando. Dependendo dos valores dos parâmetros, esse risco máximo pode ser mais no início ou no final do tempo de acompanhamento. Novamente para facilitar na visualização dos gráficos foram utilizadas escalas diferentes no eixo $y$. 

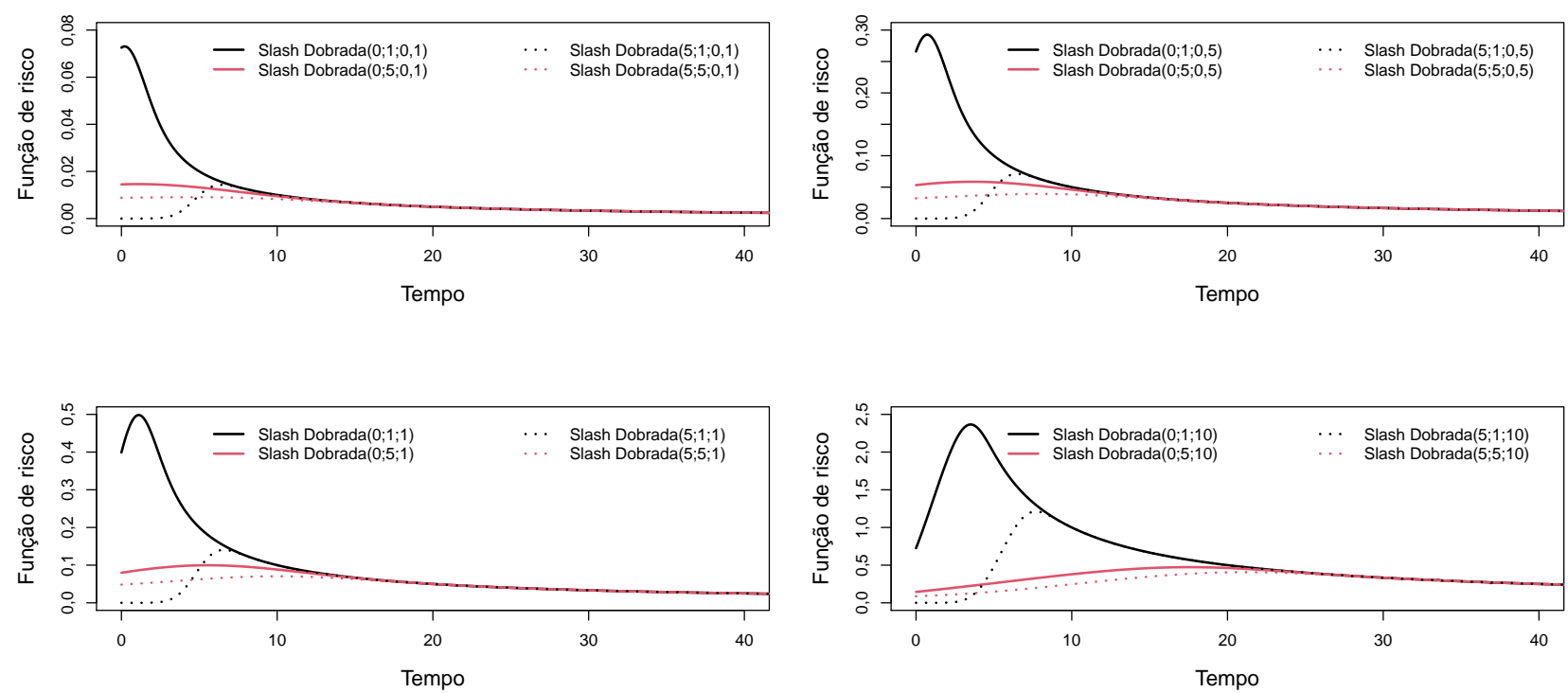

Figura 2.6: Função de risco da distribuição Slash Dobrada $(\mu, \sigma, q)$

\subsection{Identificabilidade no modelo Slash Dobrado}

A questão de identificabilidade está presente em um modelo complexo como Slash Dobrado. Considere uma amostra aleatória de tamanho $n$ da distribuição Slash Dobrada, $T_{i}, i=1, \ldots, n$, representando o tempo até um evento de interesse. Então $T_{i} \sim \operatorname{Slash} \operatorname{Dobrada}(\mu, \sigma, q), \mu \in \mathbb{R}$, $\sigma \in \mathbb{R}_{+}, q \in \mathbb{R}_{+}$, e a função de verossimilhança é dada por

$$
L(\gamma)=\prod_{i=1}^{n} f_{T_{i}}\left(t_{i}\right)=\prod_{i=1}^{n} \int_{0}^{1} \frac{q u^{q}}{\sigma \sqrt{2 \pi}}\left[e^{-\frac{\left(u t_{i}-\mu\right)^{2}}{2 \sigma^{2}}}+e^{-\frac{\left(u t_{i}+\mu\right)^{2}}{2 \sigma^{2}}}\right] \mathrm{d} u
$$

$\operatorname{com} \gamma=(\mu, \sigma, q)^{T}$.

Para encontrar os estimadores de máxima verossimilhança considera-se a função log-verossimilhança,

$$
\log (L(\gamma))=l(\gamma)=n \log q-n \log \sigma-\frac{n}{2} \log (2 \pi)+\sum_{i=1}^{n} \log \int_{0}^{1}\left[e^{-\frac{\left(u t_{i}-\mu\right)^{2}}{2 \sigma^{2}}}+e^{-\frac{\left(u t_{i}+\mu\right)^{2}}{2 \sigma^{2}}}\right] u^{q} \mathrm{~d} u
$$

Nota-se que para valores de $\mu$ com sinais opostos, mantendo-se os outros parâmetros constantes, tanto (2.7) como (2.8) levam ao mesmo valor evidenciando um problema de identificabilidade. Para contornar esse problema, sem perda de generalidade, pode-se considerar $\mu \geq 0$. Liu et al. [2020] 
consideraram essa restrição no espaço paramétrico ao utilizar a distribuição Normal Dobrada em problemas de regressão. Uma alternativa conveniente será utilizar uma reparametrização, como por exemplo $\mu=\exp \{\beta\}$. Assim $\beta \in \mathbb{R}$ e $\mu \in \mathbb{R}_{+}$, evitando-se assim eventuais problemas de fronteira nos procedimentos de estimação e testes de hipóteses. 


\section{Capítulo 3}

\section{Distribuições Normal Dobrada e Slash}

\section{Dobrada em Análise de Sobrevivência}

Neste capítulo é feita uma discussão sobre o uso das distribuições apresentadas no capítulo anterior, Normal Dobrada e Slash Dobrada, na modelagem de dados de sobrevivência. Para a utilização dessas distribuições em dados censurados são necessárias adequações e reparametrizações, que geram ganhos computacionais. Para avaliações do estimadores de máxima verossimilhança e estatísticas de testes, são realizados estudos de simulação.

\subsection{Modelagem para dados censurados}

Considere uma amostra de tamanho $n$ representada por $\left\{\left(T_{i}, C_{i}\right), i=1, \ldots, n\right\}$ em que $T_{i}, i=$ $1, \ldots, n$, representam tempos até um evento de interesse e $C_{i}, i=1, \ldots, n$, instantes de censura. As quantidades de fato observáveis são representadas pelas variáveis aleatórias $Z_{i}$ e $\delta_{i}$, em que

$$
Z_{i}=\min \left\{T_{i}, C_{i}\right\} \text { e } \delta_{i}=I\left(T_{i} \leq C_{i}\right), \operatorname{com} i=1, \ldots, n
$$

A informação, $C_{i}$ pode ser variável aleatória ou constante, dependendo do estudo.

Admitindo que os tempos de falha e de censura são independentes e a censura é não informativa (ver Klein \& Moeschberger [2005]), tem-se que a função de verossimilhança é dada por

$$
L(\gamma)=\prod_{i=1}^{n}\left[f_{T_{i}}\left(z_{i}\right)\right]^{\delta_{i}}\left[S_{T_{i}}\left(z_{i}\right)\right]^{1-\delta_{i}},
$$

com $f_{T_{i}}($.$) e S_{T_{i}}($.$) as funções densidade de probabilidade e de sobrevivência, respectivamente.$ 


\subsubsection{Tempos de falha com a distribuição Normal Dobrada}

Considerando a distribuição Normal Dobrada vista no capítulo anterior, e substituindo-se as expressões (2.1) e (2.2) em (3.1), obtém-se que a função de verossimilhança correspondente é dada por

$$
L(\gamma)=\prod_{i=1}^{n}\left\{\frac{1}{\sigma \sqrt{2 \pi}}\left[e^{-\frac{\left(z_{i}-\mu\right)^{2}}{2 \sigma^{2}}}+e^{-\frac{\left(z_{i}+\mu\right)^{2}}{2 \sigma^{2}}}\right]\right\}^{\delta_{i}}\left\{\frac{1}{\sigma \sqrt{2 \pi}} \int_{z_{i}}^{\infty}\left[e^{-\frac{(y-\mu)^{2}}{2 \sigma^{2}}}+e^{-\frac{(y+\mu)^{2}}{2 \sigma^{2}}}\right] \mathrm{d} y\right\}^{1-\delta_{i}}
$$

$\operatorname{com} \gamma=(\mu, \sigma)^{T}$.

Para encontrar os estimadores de máxima verossimilhança utiliza-se a log-verossimilhança dada por

$$
l(\gamma)=-n \log (\sigma)+\sum_{i=1}^{n} \delta_{i} \log \left(l_{1 i}\right)+\sum_{i=1}^{n}\left(1-\delta_{i}\right) \log \left(l_{2 i}\right)
$$

em que $l_{1 i}=\frac{1}{\sqrt{2 \pi}}\left[e^{-\frac{\left(z_{i}-\mu\right)^{2}}{2 \sigma^{2}}}+e^{-\frac{\left(z_{i}+\mu\right)^{2}}{2 \sigma^{2}}}\right]$ e $l_{2 i}=\frac{1}{\sqrt{2 \pi}} \int_{z_{i}}^{\infty}\left[e^{-\frac{(y-\mu)^{2}}{2 \sigma^{2}}}+e^{-\frac{(y+\mu)^{2}}{2 \sigma^{2}}}\right] \mathrm{d} y$.

A função score, $\boldsymbol{U}(\boldsymbol{\gamma})$, é obtida derivando o logaritmo da verossimilhança em relação à $\boldsymbol{\gamma}$. Desta forma

$$
\begin{aligned}
\frac{\partial l(\gamma)}{\partial \mu} & =\sum_{i=1}^{n} \delta_{i} \frac{\frac{e^{-\frac{\left(z_{i}-\mu\right)^{2}}{2 \sigma^{2}}}}{\sqrt{2 \pi}}\left(\frac{z_{i}-\mu}{\sigma^{2}}\right)-\frac{e^{-\frac{\left(z_{i}+\mu\right)^{2}}{2 \sigma^{2}}}}{\sqrt{2 \pi}}\left(\frac{z_{i}+\mu}{\sigma^{2}}\right)}{l_{1 i}}+ \\
& +\sum_{i=1}^{n}\left(1-\delta_{i}\right) \frac{\int_{z_{i}}^{\infty}\left(\frac{e^{-\frac{(y-\mu)^{2}}{2 \sigma^{2}}}}{\sqrt{2 \pi}}\left(\frac{y-\mu}{\sigma^{2}}\right)-\frac{e^{-\frac{(y+\mu)^{2}}{2 \sigma^{2}}}}{\sqrt{2 \pi}}\left(\frac{y+\mu}{\sigma^{2}}\right)\right) \mathrm{d} y}{l_{2 i}}
\end{aligned}
$$

e

$$
\begin{aligned}
\frac{\partial l(\gamma)}{\partial \sigma} & =-\frac{n}{\sigma}+\sum_{i=1}^{n} \delta_{i} \frac{\frac{e^{-\frac{\left(z_{i}-\mu\right)^{2}}{2 \sigma^{2}}}}{\sqrt{2 \pi}}\left(\frac{\left(z_{i}-\mu\right)^{2}}{\sigma^{3}}\right)+\frac{e^{-\frac{\left(z_{i}+\mu\right)^{2}}{2 \sigma^{2}}}}{\sqrt{2 \pi}}\left(\frac{\left(z_{i}+\mu\right)^{2}}{\sigma^{3}}\right)}{l_{1 i}}+ \\
& +\sum_{i=1}^{n}\left(1-\delta_{i}\right) \frac{\int_{z_{i}}^{\infty}\left(\frac{e^{-\frac{(y-\mu)^{2}}{2 \sigma^{2}}}}{\sqrt{2 \pi}}\left(\frac{(y-\mu)^{2}}{\sigma^{3}}\right)+\frac{e^{-\frac{(y+\mu)^{2}}{2 \sigma^{2}}}}{\sqrt{2 \pi}}\left(\frac{(y+\mu)^{2}}{\sigma^{3}}\right)\right) \mathrm{d} y}{l_{2 i}}
\end{aligned}
$$


Como as derivadas possuem formas complicadas não é possível encontrar analiticamente os estimadores de máxima verossimilhança. Assim, utiliza-se métodos numéricos, como o algoritmo de Newton-Raphson, que envolve as segundas derivadas, apresentadas na Seção A.1.

\subsubsection{Tempos de falha com a distribuição Slash Dobrada}

Considerando a distribuição Slash Dobrado vista no capítulo anterior, e substituindo-se as expressões (2.5) e (2.6) em (3.1), obtém-se que a função de verossimilhança

$L(\gamma)=\prod_{i=1}^{n}\left[\int_{0}^{1} \frac{q u^{q}}{\sigma \sqrt{2 \pi}}\left[e^{-\frac{\left(u z_{i}-\mu\right)^{2}}{2 \sigma^{2}}}+e^{-\frac{\left(u z_{i}+\mu\right)^{2}}{2 \sigma^{2}}}\right] \mathrm{d} u\right]^{\delta_{i}}\left[\int_{0}^{1} \frac{q u^{q}}{\sigma \sqrt{2 \pi}} \int_{z_{i}}^{\infty}\left[e^{-\frac{(u y-\mu)^{2}}{2 \sigma^{2}}}+e^{-\frac{(u y+\mu)^{2}}{2 \sigma^{2}}}\right] \mathrm{d} y \mathrm{~d} u\right]^{1-\delta_{i}}$,

com $\gamma=(\mu, \sigma, q)^{T}$, e a correspondente log-verossimilhança

$$
l(\gamma)=n \log \left(\frac{q}{\sigma}\right)+\sum_{i=1}^{n} \delta_{i} \log \left(l_{1 i}\right)+\sum_{i=1}^{n}\left(1-\delta_{i}\right) \log \left(l_{2 i}\right),
$$

em que $l_{1 i}=\int_{0}^{1} \frac{u^{q}}{\sqrt{2 \pi}}\left[e^{-\frac{\left(u z_{i}-\mu\right)^{2}}{2 \sigma^{2}}}+e^{-\frac{\left(u z_{i}+\mu\right)^{2}}{2 \sigma^{2}}}\right] \mathrm{d} u$ e $l_{2 i}=\int_{0}^{1} \frac{u^{q}}{\sqrt{2 \pi}} \int_{z_{i}}^{\infty}\left[e^{-\frac{(u y-\mu)^{2}}{2 \sigma^{2}}}+e^{-\frac{(u y+\mu)^{2}}{2 \sigma^{2}}}\right] \mathrm{d} y \mathrm{~d} u$.

A função score, $\boldsymbol{U}(\boldsymbol{\gamma})$, é obtida derivando o logaritmo da verossimilhança em relação à $\gamma$. Desta forma,

$$
\begin{aligned}
\frac{\partial l(\gamma)}{\partial \mu} & =\sum_{i=1}^{n} \delta_{i} \frac{\int_{0}^{1} \frac{u^{q}}{\sqrt{2 \pi}}\left(e^{-\frac{\left(u z_{i}-\mu\right)^{2}}{2 \sigma^{2}}}\left(\frac{u z_{i}-\mu}{\sigma^{2}}\right)+e^{-\frac{\left(u z_{i}+\mu\right)^{2}}{2 \sigma^{2}}}\left(-\frac{u z_{i}+\mu}{\sigma^{2}}\right)\right) \mathrm{d} u}{l_{1 i}}+ \\
& +\sum_{i=1}^{n}\left(1-\delta_{i}\right) \frac{\int_{0}^{1} \int_{z_{i}}^{\infty} \frac{u^{q}}{\sqrt{2 \pi}}\left(e^{-\frac{(u y-\mu)^{2}}{2 \sigma^{2}}}\left(\frac{u y-\mu}{\sigma^{2}}\right)+e^{-\frac{(u y+\mu)^{2}}{2 \sigma^{2}}}\left(-\frac{u y+\mu}{\sigma^{2}}\right)\right) \mathrm{d} y \mathrm{~d} u}{l_{2 i}}, \\
\frac{\partial l(\boldsymbol{\gamma})}{\partial \sigma} & =-\frac{n}{\sigma}+\sum_{i=1}^{n} \delta_{i} \frac{\int_{0}^{1} \frac{u^{q}}{\sqrt{2 \pi}}\left(e^{-\frac{\left(u z_{i}-\mu\right)^{2}}{2 \sigma^{2}}}\left(\frac{\left(u z_{i}-\mu\right)^{2}}{\sigma^{3}}\right)+e^{-\frac{\left(u z_{i}+\mu\right)^{2}}{2 \sigma^{2}}}\left(\frac{\left(u z_{i}+\mu\right)^{2}}{\sigma^{3}}\right)\right) \mathrm{d} u}{l_{1 i}}+ \\
& +\sum_{i=1}^{n}\left(1-\delta_{i}\right) \frac{\int_{0}^{1} \int_{z_{i}}^{\infty} \frac{u^{q}}{\sqrt{2 \pi}}\left(e^{-\frac{(u y-\mu)^{2}}{2 \sigma^{2}}}\left(\frac{(u y-\mu)^{2}}{\sigma^{3}}\right)+e^{-\frac{(u y+\mu)^{2}}{2 \sigma^{2}}}\left(\frac{(u y+\mu)^{2}}{\sigma^{3}}\right)\right) \mathrm{d} y \mathrm{~d} u}{l_{2 i}}
\end{aligned}
$$




$$
\begin{aligned}
\frac{\partial l(\gamma)}{\partial q} & =-\frac{n}{q}+\sum_{i=1}^{n} \delta_{i} \frac{\int_{0}^{1} \frac{u^{q} \log (u)}{\sqrt{2 \pi}}\left(e^{-\frac{\left(u z_{i}-\mu\right)^{2}}{2 \sigma^{2}}}+e^{-\frac{\left(u z_{i}+\mu\right)^{2}}{2 \sigma^{2}}}\right) \mathrm{d} u}{l_{1 i}}+ \\
& +\sum_{i=1}^{n}\left(1-\delta_{i}\right) \frac{\int_{0}^{1} \int_{z_{i}}^{\infty} \frac{u^{q} \log (u)}{\sqrt{2 \pi}}\left(e^{-\frac{(u y-\mu)^{2}}{2 \sigma^{2}}}+e^{-\frac{(u y+\mu)^{2}}{2 \sigma^{2}}}\right) \mathrm{d} y \mathrm{~d} u}{l_{2 i}}
\end{aligned}
$$

Como no caso da Normal Dobrada não é possível encontrar expressões explícitas para os estimadores de máxima verossimilhança, sendo necessário o uso de métodos numéricos. Para o algoritmo de Newton-Raphson é necessário encontrar as segundas derivadas, apresentadas na Seção A.2.

Alternativamente, utilizando-se apenas as primeiras derivadas, pode-se encontrar os estimadores de máxima verossimilhança considerando o método desenvolvido por Broyden, Fletcher, Goldfarb e Shanno (BFGS), Broyden [1965], no algoritmo de quasi Newton, para mais detalhes ver Ribeiro \& Karas [2013].

\section{Algoritmo de Newton-Raphson}

Conforme mencionado acima, o algoritmo de Newton-Raphson utiliza as funções score, $\boldsymbol{U}(\boldsymbol{\gamma})$, e a informação observada, $-\boldsymbol{I}(\boldsymbol{\gamma})$. O algoritmo atualiza $\gamma$ na etapa $j$ utilizando os valores na etapa $j-1$ através da expressão

$$
\left.\begin{array}{c}
\boldsymbol{\gamma}^{(j)}=\boldsymbol{\gamma}^{(j-1)}-\boldsymbol{U}\left(\boldsymbol{\gamma}^{(j-1)}\right) \boldsymbol{I}^{-1}\left(\boldsymbol{\gamma}^{(j-1)}\right), \\
\frac{\partial l(\boldsymbol{\gamma})}{\partial \sigma} \\
\frac{\partial l(\boldsymbol{\gamma})}{\partial q}
\end{array}\right) \text { e } \boldsymbol{I}(\boldsymbol{\gamma})=\left(\begin{array}{ccc}
\frac{\partial^{2} l(\boldsymbol{\gamma})}{\partial \mu^{2}} & \frac{\partial^{2} l(\boldsymbol{\gamma})}{\partial \mu \partial \sigma} & \frac{\partial^{2} l(\boldsymbol{\gamma})}{\partial \mu \partial q} \\
\frac{\partial^{2} l(\boldsymbol{\gamma})}{\partial \mu \partial \sigma} & \frac{\partial^{2} l(\boldsymbol{\gamma})}{\partial \sigma^{2}} & \frac{\partial^{2} l(\boldsymbol{\gamma})}{\partial \sigma \partial q} \\
\frac{\partial^{2} l(\boldsymbol{\gamma})}{\partial \mu \partial q} & \frac{\partial^{2} l(\boldsymbol{\gamma})}{\partial \sigma \partial q} & \frac{\partial^{2} l(\boldsymbol{\gamma})}{\partial q^{2}}
\end{array}\right) .
$$

Repete-se o algoritmo até a convergência. Como critério de parada será considerado

$$
\left(\left(\gamma^{(j)}-\gamma^{(j-1)}\right)^{T}\left(\gamma^{(j)}-\gamma^{(j-1)}\right)\right)^{1 / 2}<10^{-6}
$$




\subsection{Estudo de simulação}

Para verificar o comportamento dos estimadores de máxima verossimilhança dos parâmetros do modelo Slash Dobrado foi realizado um estudo de simulação implementado na linguagem R.

A fim de evitar problemas de identificabilidade e de fronteira foram consideradas no modelo 3.2 as reparametrizações $\mu=\exp \left\{\beta_{\mu}\right\}$ e $q=\exp \left\{\beta_{q}\right\}$, o que também possibilita incluir covariáveis nos dois parâmetros e evita restrições no espaço paramétrico, simplificando o algoritmo computacional.

Na geração dos dados foi utilizada a independência entre as distribuições Normal Dobrada e Beta, e gerados valores das distribuições Normal, com parâmetros $\mu$ e $\sigma^{2}$, e Beta, com parâmetros $q$ e 1 , tomado o módulo do quociente como na expressão 2.4 , resultando em observações, $T_{i}, i=1, \ldots, n$, de uma distribuição Slash Dobrada, com parâmetros $\mu, \sigma$ e $q$.

Foram geradas amostras de tamanho 100, 500 e 1.000. Nestes cenários considerou-se casos que tinham somente censura do tipo I, aleatória, e de ambos os tipos. Após a geração dos tempos de falhas, $T_{i}, i=1, \ldots, n$, foram geradas as censuras.

Para as censuras aleatórias foi utilizada a distribuição Bernoulli, digamos $W$, com a proporção de censura de interesse.

$$
W= \begin{cases}1, & \text { o tempo é de falha, } \quad \Rightarrow \quad Z_{i}=t_{i} \\ 0, & \text { o tempo é de censura, } \Rightarrow Z_{i}=C_{i}\end{cases}
$$

Se $w=1$ o tempo obtido anteriormente é utilizado como tempo de falha. Se $w=0$ deve-se gerar um novo valor que será um tempo de censura.

$$
C_{i} \sim \text { Uniforme }\left(0, \min \left\{\tau, t_{i}\right\}\right)
$$

O valor $\tau$ é determinado considerando o valor de corte do experimento o qual deixa um percentual $k$ de dados à direita desse valor, ou seja $\mathbb{P}(T>\tau)=k \%$, sendo $T \sim \operatorname{Slash} \operatorname{Dobrada}(\mu, \sigma, q)$. Os tempos de falhas obtidos anteriormente que eram superiores a $\tau$ foram censurados e atribuído o valor de $\tau$ para este tempo. Com isso obtém-se os tempos de falha e de censura. Em média o percentual de censura geral, aleatória mais tipo I, é o valor pré fixado.

Para facilitar a programação foi utilizado o artifício de expressar as derivadas obtidas nas funções score e informação, $U($.$) e I($.$) respectivamente, em função de densidades da distribuição Normal e$ de sobrevivência das distribuições Normal e Gama, assim como as expressões (2.5) e (2.6), e utilizar as funções intrínsecas do software R. Com isso, houve um ganho computacional e o programa convergiu mais rapidamente, uma vez que não foi necessário tratar diretamente integrais duplas. 
Fazendo transformações afim de expressar as derivadas obtidas em função do núcleo das distribuições Normal e Gama foi possível simplificar as derivadas. O ganho no tempo computacional foi bastante considerável, reduzindo de horas para minutos em alguns casos.

Conforme mencionado em Tsagris et al. [2014], verificou-se que o valor inicial no processo iterativo deve levar em consideração a relação entre $\mu$ e $\sigma$. Considera-se assim valores iniciais com $\mu$ maior que $\sigma$ e $\mu$ menor que $\sigma$. Verificou-se que mesmo que os valores iniciais estejam distantes dos verdadeiros valores usados para gerar os dados, o algoritmo se comporta de forma satisfatória, desde que essa relação seja mantida. Caso contrário, o procedimento pode se tornar bastante instável.

Com esse critério, considerando as réplicas em que o algoritmo convergiu, foram necessárias poucas iterações. Na Tabela 3.1 encontram-se alguns valores para verificar o número de iterações necessárias até a convergência do algoritmo. Em todos os casos foram consideradas 100 réplicas.

Tabela 3.1: Número de iterações até a convergência do algoritmo.

\begin{tabular}{cccc}
\hline \hline Percentual de censura & $5 \%$ & $25 \%$ & $50 \%$ \\
\hline Média de iterações & 7 & 18 & 26 \\
Mínimo de iterações & 4 & 5 & 11 \\
Máximo de iterações & 9 & 50 & 50 \\
\hline \hline
\end{tabular}

Conforme o percentual de censura aumentou foram necessárias um pouco mais de iterações. No entanto, o valor máximo de iterações não ultrapassou 50.

Para verificar o efeito da censura seria interessante utilizar uma distribuição Slash Dobrada com o mesmo conjunto de parâmetros. No entanto, uma distribuição com cauda mais pesada admite a probabilidade de valores muito grandes, e a geração destes valores causa problemas na convergência das integrais. É interessante notar que quando existe censura tipo I, como estes valores maiores são censurados, este problema é corrigido. Assim para percentuais pequenos de censura tornase complicado utilizar uma distribuição com cauda mais pesada. Já para percentuais maiores de censura surge a necessidade de uma cauda mais pesada e uma maior assimetria. Assim optou-se por utilizar uma distribuição com cauda mais leve e mais simétrica para percentuais menores de censura e uma distribuição com cauda mais pesada e maior assimetria para percentuais maiores de censura.

Para os casos com percentuais de censura baixo foi considerada a distribuição Slash Dobrada(16, 4, 3). Neste caso, existe a esperança e o desvio padrão, sendo iguais, respectivamente, a 24,0 e 27,9. A mediana é igual a 20,9, o que evidencia uma pequena assimetria. Já para percentuais maiores de 
censura foi considerada a distribuição Slash Dobrada $(2 ; 80 ; 0,5)$; neste caso tanto a média como o desvio padrão não existem, mas a mediana é igual a 216,0.

A Tabela 3.2 apresenta resultados da simulação considerando $5 \%$ de censura, em três cenários considerando combinações diferentes de censuras do tipo I e aleatória. Nestes cenários, verifica-se que conforme o tamanho das amostras aumenta as estimativas dos parâmetros se aproximam dos verdadeiros valores, como esperado. Nota-se ainda que para percentuais de censura mais baixos não faz muita diferença como se dá a composição das censuras.

Tabela 3.2: Estimativas para os parâmetros da distribuição Slash Dobrada $(16,4,3)$ com $5 \%$ de censura e 100 réplicas de tamanhos 100, 500 e 1.000.

\begin{tabular}{|c|c|c|c|c|c|c|c|c|c|c|}
\hline \multirow{2}{*}{$\begin{array}{l}\text { Tipo de } \\
\text { Censura }\end{array}$} & & \multicolumn{3}{|c|}{$n=100$} & \multicolumn{3}{|c|}{$n=500$} & \multicolumn{3}{|c|}{$n=1.000$} \\
\hline & & $\hat{\mu}$ & $\hat{\sigma}$ & $\hat{q}$ & $\hat{\mu}$ & $\hat{\sigma}$ & $\hat{q}$ & $\hat{\mu}$ & $\hat{\sigma}$ & $\hat{q}$ \\
\hline $0 \%$ & Média & 15,88 & 3,74 & 3,01 & 15,92 & 3,94 & 3,00 & 15,94 & 3,91 & 2,99 \\
\hline aleatória & Erro padrão & 0,85 & 0,66 & 0,54 & 0,40 & 0,29 & 0,27 & 0,33 & 0,22 & 0,20 \\
\hline$+5 \%$ & Vício & $-0,12$ & $-0,26$ & 0,01 & $-0,08$ & $-0,06$ & $-0,01$ & $-0,06$ & $-0,09$ & $-0,01$ \\
\hline tipo I & EQM & 0,73 & 0,50 & 0,29 & 0,17 & 0,09 & 0,07 & 0,12 & 0,05 & 0,04 \\
\hline $2,5 \%$ & Média & 15,79 & 3,63 & 2,95 & 15,84 & 3,83 & 2,93 & 15,86 & 3,81 & 2,93 \\
\hline aleatória & Erro padrão & 0,79 & 0,62 & 0,49 & 0,37 & 0,28 & 0,24 & 0,33 & 0,21 & 0,18 \\
\hline$+2,5 \%$ & Vício & $-0,21$ & $-0,37$ & $-0,05$ & $-0,16$ & $-0,17$ & $-0,07$ & $-0,14$ & $-0,19$ & $-0,07$ \\
\hline tipo I & EQM & 0,67 & 0,52 & 0,24 & 0,16 & 0,10 & 0,06 & 0,13 & 0,08 & 0,04 \\
\hline $5 \%$ & Média & 15,88 & 3,74 & 3,01 & 15,92 & 3,94 & 3,00 & 15,94 & 3,91 & 2,99 \\
\hline aleatória & Erro padrão & 0,85 & 0,66 & 0,54 & 0,40 & 0,29 & 0,27 & 0,33 & 0,22 & 0,20 \\
\hline$+0 \%$ & Vício & $-0,12$ & $-0,26$ & 0,01 & $-0,08$ & $-0,06$ & $-0,01$ & $-0,06$ & $-0,09$ & $-0,01$ \\
\hline tipo I & EQM & 0,73 & 0,50 & 0,29 & 0,17 & 0,09 & 0,07 & 0,12 & 0,05 & 0,04 \\
\hline
\end{tabular}

Para se ter uma ideia do comportamento assintótico dos estimadores de máxima verossimilhança serão utilizadas as estimativas de $\beta_{\mu}$ e $\beta_{q}$. Considerando que os valores de $\mu=16$ tem-se $\beta_{\mu}=2,77$ e para $q=3$, tem-se $\beta_{q}=1,10$.

Pelas Figuras 3.1, 3.2 e 3.3 é possível notar que conforme o tamanho amostral aumenta os histogramas das estimativas dos parâmetros vão ficando com aparência da distribuição Normal, sugerindo que as propriedades assintóticas usuais dos estimadores de máxima verossimilhança são válidas. Nas figuras, a linha vertical em vermelho indica o verdadeiro valor do parâmetro, e em geral é próxima ao ponto de simetria dos gráficos. 

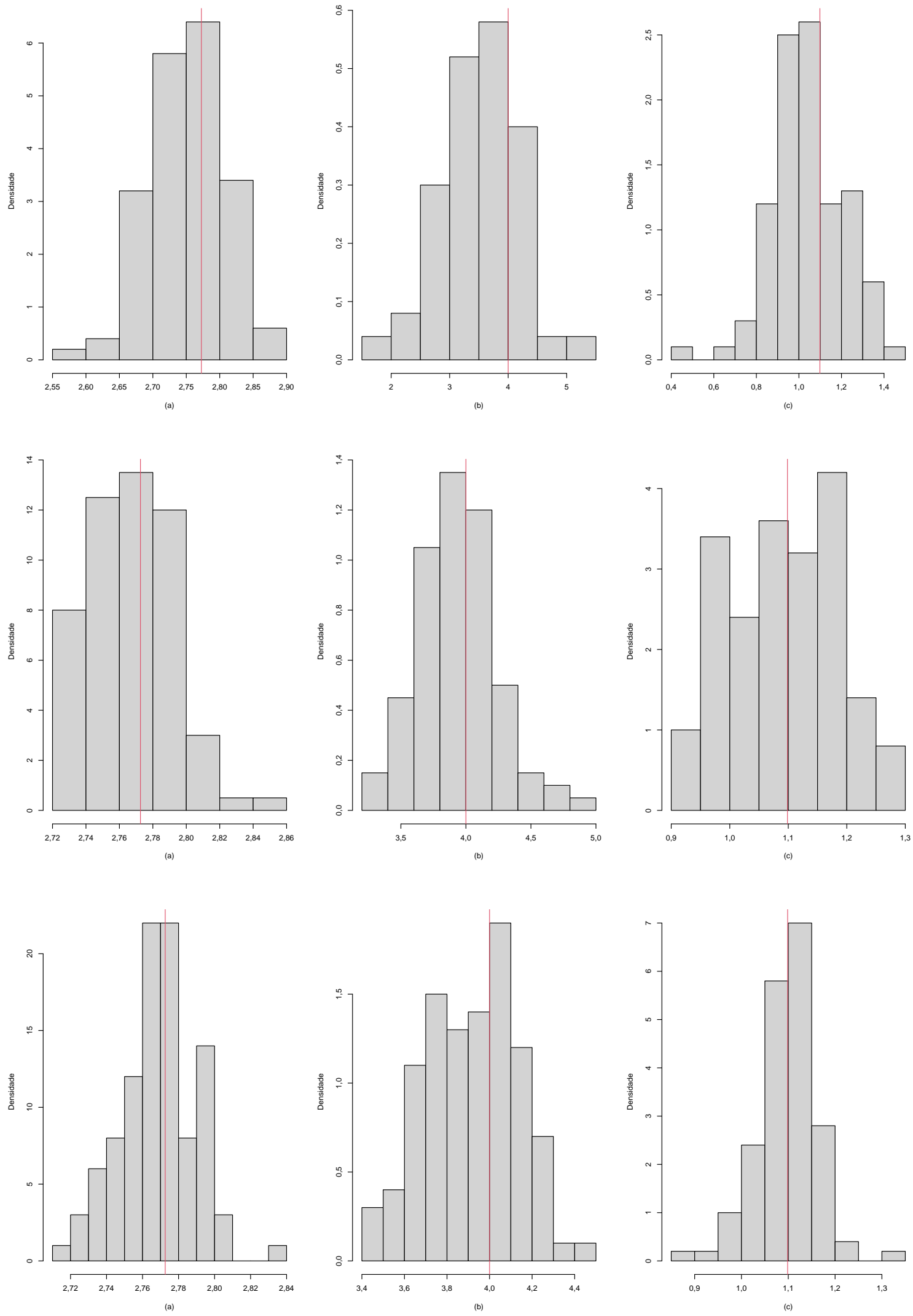

Figura 3.1: Histogramas das estimativas dos parâmetros da distribuição Slash Dobrada(exp $(2,77)$, $4, \exp (1,10))$. Nas colunas tem-se os parâmetros, (a) $\beta_{\mu}$, (b) $\sigma$ e (c) $\beta_{q}$, com $0 \%$ de censura aleatória e 5\% tipo I em 100 réplicas. Nas linhas tem-se os tamanhos amostrais, 100, 500 e 1.000. 

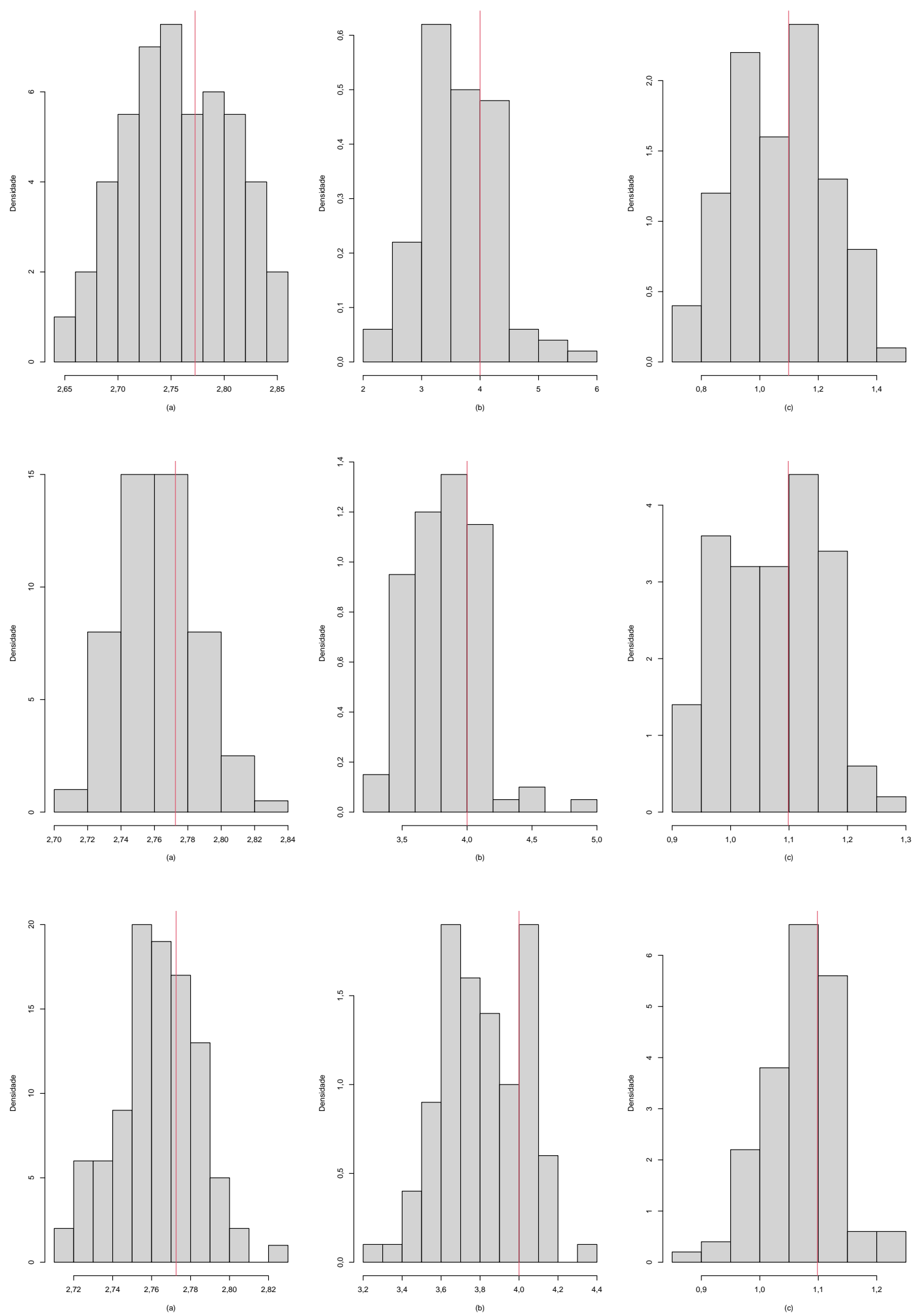

Figura 3.2: Histogramas das estimativas dos parâmetros da distribuição Slash Dobrada( $\exp (2,77)$, $4, \exp (1,10))$. Nas colunas tem-se os parâmetros, (a) $\beta_{\mu}$, (b) $\sigma e$ (c) $\beta_{q}$, com $2,5 \%$ de censura aleatória e 2,5\% tipo I em 100 réplicas. Nas linhas tem-se os tamanhos amostrais, 100, 500 e 1.000. 

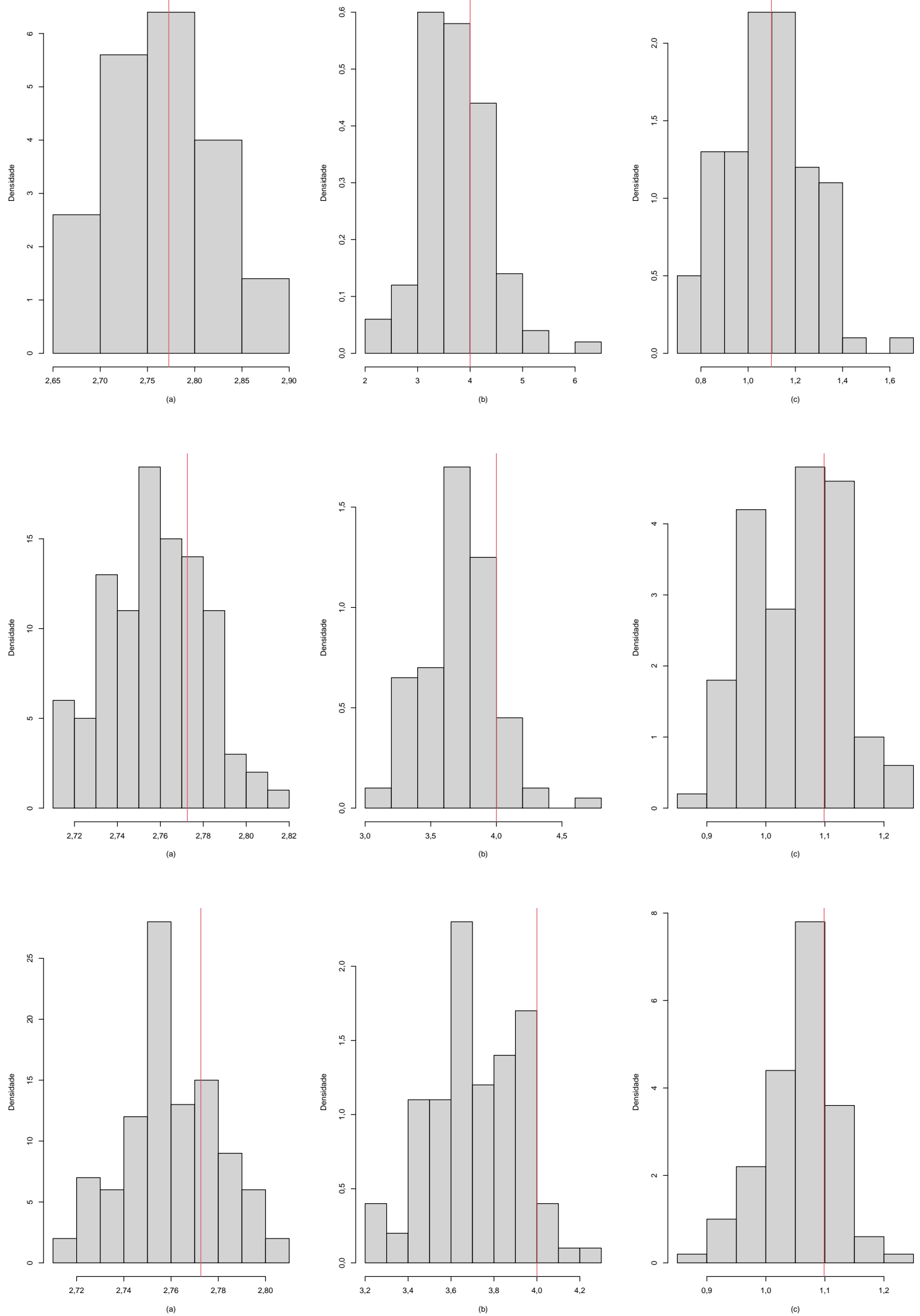

Figura 3.3: Histogramas das estimativas dos parâmetros da distribuição Slash Dobrada(exp $(2,77)$, $4, \exp (1,10))$. Nas colunas tem-se os parâmetros, (a) $\beta_{\mu}$, (b) $\sigma$ e (c) $\beta_{q}$, com $5 \%$ de censura aleatória e 0\% tipo I em 100 réplicas. Nas linhas tem-se os tamanhos amostrais, 100, 500 e 1.000. 
Para maiores concentrações de censura é necessário maior assimetria, assim uma cauda mais pesada é mais interessante. Para analisar cenários com $25 \%$ de censura é considerada a distribuição Slash Dobrada $(2 ; 80 ; 0,5)$, que consegue acomodar de forma satisfatória tanto $25 \%$ quanto $50 \%$ de censura, como será visto a seguir.

Na Tabela 3.3 são apresentados os cenários com $25 \%$ de censura. Verifica-se que ocorre uma superestimação do parâmetro $\mu$; para os parâmetros $\sigma$ e $q$ as estimativas tendem a se aproximar dos verdadeiros valores conforme o tamanho amostral aumenta, enquanto que o erro padrão diminui.

Tabela 3.3: Estimativas para os parâmetros da distribuição Slash Dobrada $(2 ; 80 ; 0,5)$ com $25 \%$ de censura, em 100 réplicas de tamanho igual a 100, 500 e 1.000.

\begin{tabular}{|c|c|c|c|c|c|c|c|c|c|c|}
\hline \multirow{2}{*}{$\begin{array}{l}\text { Tipo de } \\
\text { Censura }\end{array}$} & & \multicolumn{3}{|c|}{$n=100$} & \multicolumn{3}{|c|}{$n=500$} & \multicolumn{3}{|c|}{$n=1.000$} \\
\hline & & $\hat{\mu}$ & $\hat{\sigma}$ & $\hat{q}$ & $\hat{\mu}$ & $\hat{\sigma}$ & $\hat{q}$ & $\hat{\mu}$ & $\hat{\sigma}$ & $\hat{q}$ \\
\hline $0 \%$ & Média & 3,58 & 77,83 & 0,50 & 4,03 & 78,58 & 0,50 & 4,51 & 79,22 & 0,50 \\
\hline aleatória & Erro padrão & 1,98 & 19,36 & 0,10 & 0,77 & 8,19 & 0,04 & 0,78 & 5,23 & 0,03 \\
\hline$+25 \%$ & Vício & 1,58 & $-2,17$ & $-0,01$ & 2,03 & $-1,42$ & $<0,01$ & 2,51 & $-0,78$ & $<0,01$ \\
\hline tipo I & EQM & 6,40 & 375,91 & $<0,01$ & 4,70 & 68,50 & $<0,01$ & 6,93 & 27,68 & $<0,01$ \\
\hline $10 \%$ & Média & 4,03 & 84,05 & 0,48 & 4,91 & 83,11 & 0,48 & 5,44 & 83,37 & 0,48 \\
\hline aleatória & Erro padrão & 1,27 & 18,51 & 0,09 & 0,99 & 8,27 & 0,03 & 0,86 & 6,74 & 0,03 \\
\hline$+15 \%$ & Vício & 2,03 & 4,05 & $-0,02$ & 2,91 & 3,11 & $-0,02$ & 3,44 & 3,37 & $-0,02$ \\
\hline tipo I & EQM & 5,71 & 355,71 & $<0,01$ & 9,43 & 77,37 & $<0,01$ & 12,55 & 56,31 & $<0,01$ \\
\hline
\end{tabular}

Para a avaliação do comportamento assintótico dos estimadores de máxima verossimilhança nesse caso considerou-se valores de $\mu=2$ de tal forma que $\beta_{\mu}=0,69$ e $q=0,5$ que resulta $\beta_{q}=-0,69$. As Figuras 3.4 e 3.5 apresentam os histogramas das estimativas dos parâmetros, que vão concentrando e ficando com aparência da curva da distribuição Normal à medida que o tamanho amostral aumenta. Para o parâmetro $\mu$ verifica-se novamente o problema de superestimação. Em alguns histogramas a linha vertical não aparece, isso ocorre devido a superestimação nas estimativas de máxima verossimilhança. Nestes caso o valor considerado na geração dos dados fica abaixo das estimativas obtidas e assim não aparece nos histogramas. 

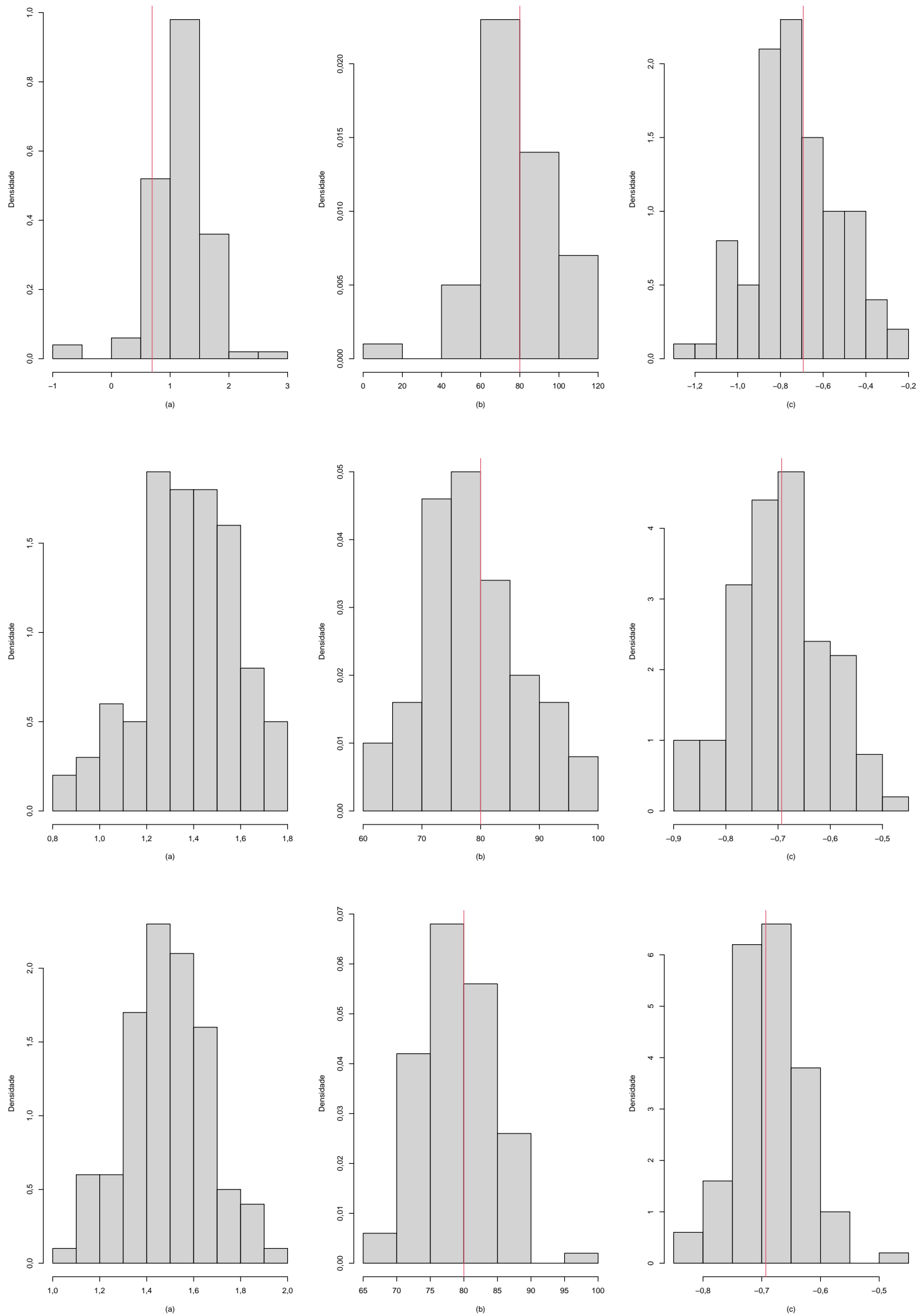

Figura 3.4: Histogramas das estimativas dos parâmetros da distribuição Slash Dobrada(exp(0,69), $80, \exp (-0,69))$. Nas colunas tem-se os parâmetros, (a) $\beta_{\mu}$, (b) $\sigma$ e (c) $\beta_{q}$, com 0\% de censura aleatória e 25\% tipo I em 100 réplicas. Nas linhas tem-se os tamanhos amostrais, 100, 500 e 1.000. 

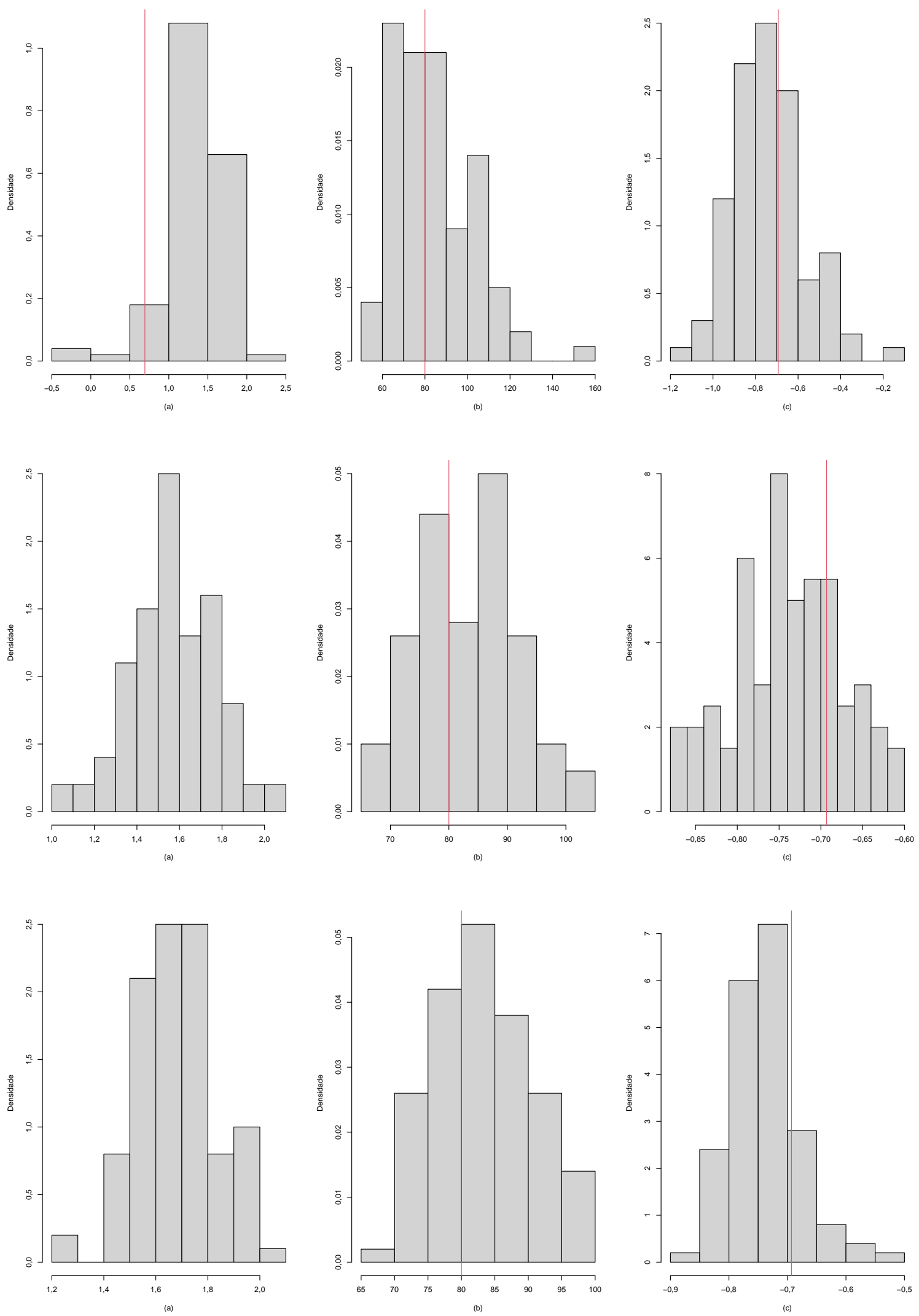

Figura 3.5: Histogramas das estimativas dos parâmetros da distribuição Slash Dobrada $(\exp (0,69)$, $80, \exp (-0,69))$. Nas colunas tem-se os parâmetros, (a) $\beta_{\mu}$, (b) $\sigma$ e (c) $\beta_{q}$, com $10 \%$ de censura aleatória e 15\% tipo I em 100 réplicas. Nas linhas tem-se os tamanhos amostrais, 100, 500 e 1.000. 
Na Tabela 3.4 tem-se os cenários com 50\% de censura. Verifica-se que o algoritmo está fornecendo estimativas próximas ao verdadeiro valor para o parâmetro $q$. No entanto, para os outros parâmetros ocorrem problemas de superestimação. Assim como os outros casos, o erro padrão diminui conforme o tamanho amostral aumenta.

Tabela 3.4: Estimativas para os parâmetros da distribuição Slash Dobrada $(2 ; 80 ; 0,5)$ com $50 \%$ de censura, em 100 réplicas de tamanho igual a 100, 500 e 1.000.

\begin{tabular}{clcccccccccc}
\hline \hline Tipo de & & \multicolumn{3}{c}{$n=100$} & \multicolumn{3}{c}{$n=500$} & \multicolumn{3}{c}{$n=1.000$} \\
Censura & & $\hat{\mu}$ & $\hat{\sigma}$ & $\hat{q}$ & $\hat{\mu}$ & $\hat{\sigma}$ & $\hat{q}$ & $\hat{\mu}$ & $\hat{\sigma}$ & $\hat{q}$ \\
\hline $0 \%$ & Média & 2,02 & 87,44 & 0,59 & 2,02 & 88,11 & 0,56 & 2,23 & 86,47 & 0,55 \\
aleatória & Erro padrão & 0,96 & 34,91 & 0,32 & 0,88 & 15,40 & 0,10 & 1,00 & 8,58 & 0,06 \\
$+50 \%$ & Vício & 0,02 & 7,44 & 0,09 & 0,02 & 8,11 & 0,06 & 0,23 & 6,47 & 0,05 \\
tipo I & EQM & 0,90 & $1.261,68$ & 0,11 & 0,77 & 300,66 & 0,01 & 1,04 & 114,63 & $<0,01$ \\
\hline $25 \%$ & Média & 3,22 & 90,66 & 0,44 & 4,49 & 90,60 & 0,43 & 4,73 & 91,99 & 0,44 \\
aleatória & Erro padrão & 1,17 & 26,90 & 0,12 & 0,65 & 10,63 & 0,04 & 0,50 & 7,47 & 0,03 \\
$+25 \%$ & Vício & 1,22 & 10,66 & $-0,06$ & 2,49 & 10,60 & $-0,07$ & 2,73 & 11,99 & $-0,06$ \\
tipo I & EQM & 2,84 & 829,85 & 0,02 & 6,64 & 224,30 & $<0,01$ & 7,71 & 198,93 & $<0,01$ \\
\hline \hline
\end{tabular}

Nas Figuras 3.6 e 3.7 visualiza-se novamente o problema da superestimação. Nota-se ainda que em determinadas situações os histogramas das estimativas do parâmetro $\mu$ não possuem a forma da distribuição Normal. Isso mostra o quão danoso pode ser altas concentrações de censura. 

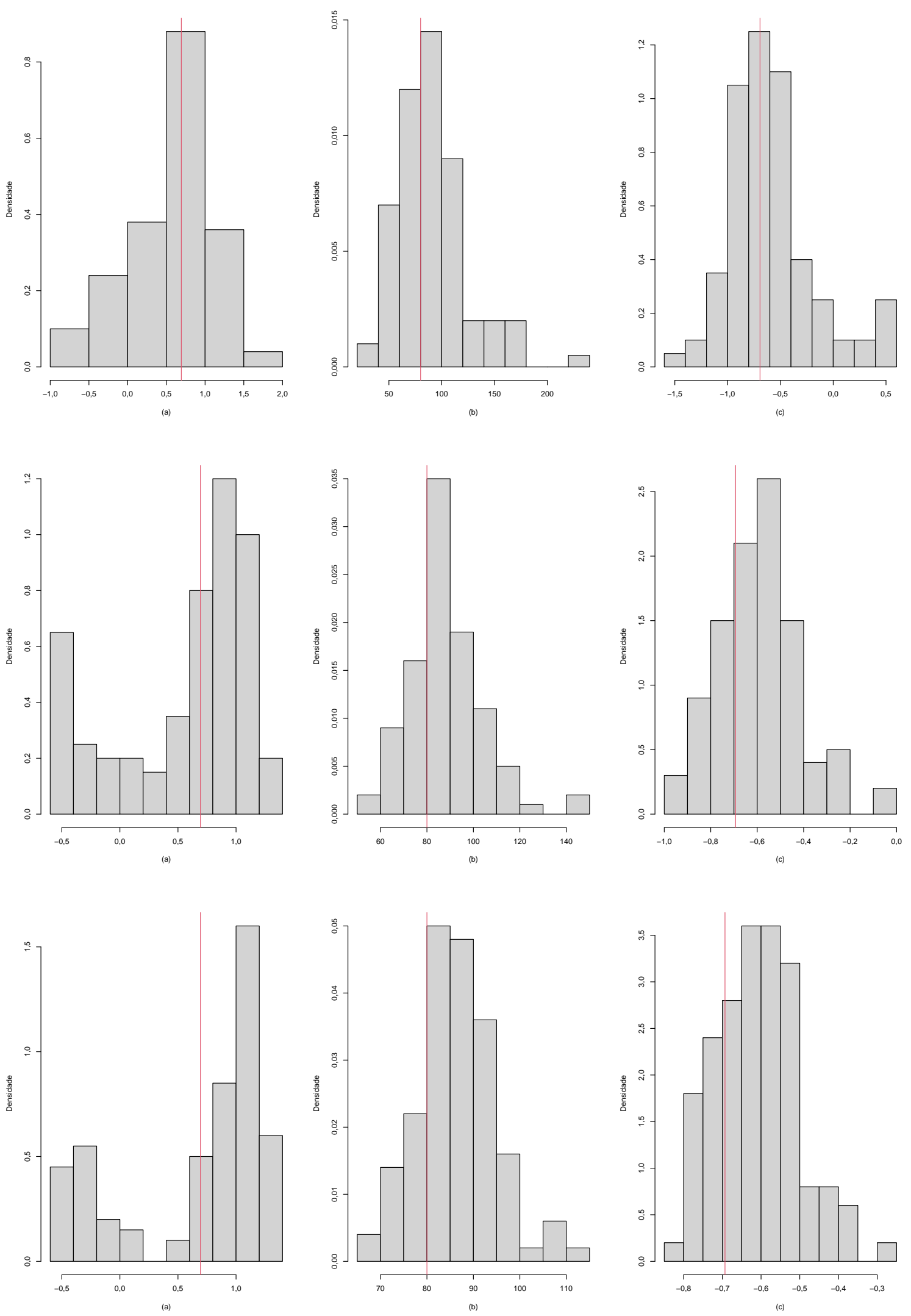

Figura 3.6: Histogramas das estimativas dos parâmetros da distribuição Slash Dobrada $(\exp (0,69)$, $80, \exp (-0,69))$. Nas colunas tem-se os parâmetros, (a) $\beta_{\mu}$, (b) $\sigma$ e (c) $\beta_{q}$, com $0 \%$ de censura aleatória e 50\% tipo I em 100 réplicas. Nas linhas tem-se os tamanhos amostrais, 100, 500 e 1.000. 

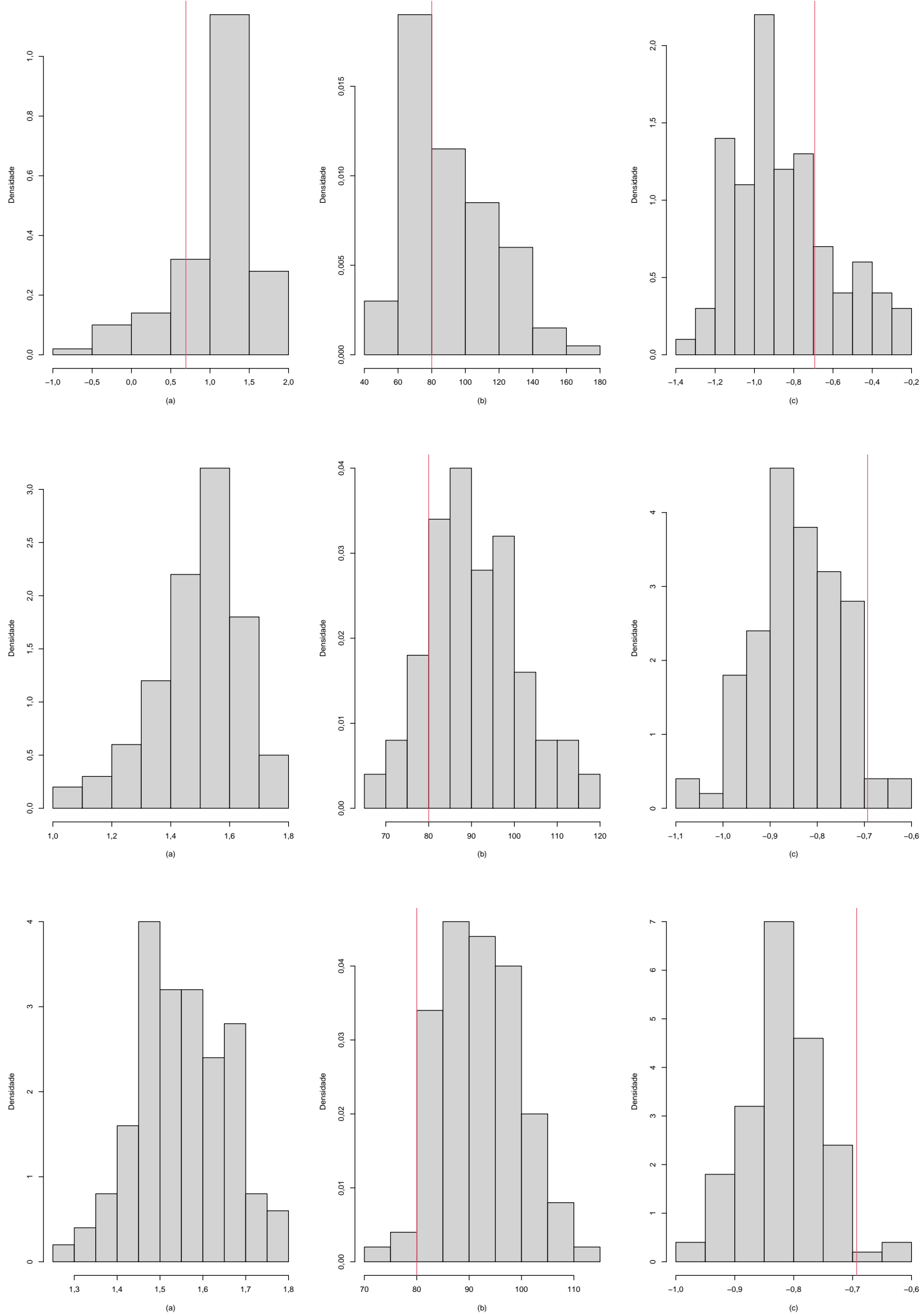

Figura 3.7: Histogramas das estimativas dos parâmetros da distribuição Slash Dobrada( $\exp (0,69)$, $80, \exp (-0,69))$. Nas colunas tem-se os parâmetros, (a) $\beta_{\mu}$, (b) $\sigma$ e (c) $\beta_{q}$, com $25 \%$ de censura aleatória e $25 \%$ tipo I em 100 réplicas. Nas linhas tem-se os tamanhos amostrais, 100, 500 e 1.000. 
Após análise desses cenários e de outros que não foram apresentados aqui, verificou-se que o procedimento tende a superestimar as estimativas dos parâmetros $\mu$ e $\sigma$ quando a distribuição possui cauda mais pesada e a proporção de censura aumenta. Quanto à estimativa do parâmetro $q$, no geral o procedimento tende a acertar as estimativas. Conforme o tamanho amostral aumenta o erro do processo iterativo tende a diminuir.

Foram analisados mais cenários do que os expostos neste trabalho, considerando variações nos parâmetros e concentrações diferentes de censura. Verificou-se que, dependendo dos valores dos parâmetros, o modelo consegue lidar melhor com altos percentuais de censura. Quando o valor de $q$ diminui a distribuição acomoda melhor um percentual de censura maior. Para valores maiores do parâmetro $q$ necessita de valores maiores de $\sigma$. Isso ocorre pois o parâmetros $q$ e $\sigma$ controlam o peso da cauda e a dispersão da distribuição. 


\subsection{Teste da razão de verossimilhanças para o parâmetro $q$}

Ao estudar o modelo Slash Dobrado, surge uma pergunta natural: "o parâmetro $q$ é pequeno ou grande?", ou de uma forma mais interessante "o modelo Slash Dobrado é adequado?". Para responder esta pergunta uma forma é propor um teste que diga se os dados são provenientes de uma distribuição Slash Dobrada ou de uma distribuição Normal Dobrada.

Ao considerar o fato de $U=W^{1 / q}$, com $U \sim \operatorname{Beta}(q, 1)$ e $W \sim \operatorname{Uniforme}(0,1)$ a distribuição Slash Dobrada é obtida através da transformação

$$
T=\frac{X}{W^{1 / q}},
$$

com $X \sim$ Nomal Dobrada.

Pode-se verificar que $\lim _{q \rightarrow \infty} W^{1 / q} \stackrel{\mathcal{P}}{\rightarrow} 1$. Assim, $T \stackrel{\mathcal{D}}{\rightarrow} X$, com $\stackrel{\mathcal{P}}{\rightarrow}$ indicando convergência em probabilidade e $\stackrel{\mathcal{D}}{\rightarrow}$ indicando convergência em distribuição. Dessa forma conforme o valor de $q$ aumenta a distribuição Slash Dobrada tende à distribuição Normal Dobrada. Analogamente ao se considerar $\xi=1 / q$, quando $\xi$ tende a zero a distribuição Slash Dobrada tende à distribuição Normal Dobrada.

Torna-se então natural verificar as hipóteses

$$
\left\{\begin{array}{l}
H_{0}: \text { O modelo Slash Dobrado pode ser reduzido ao modelo Normal Dobrado; } \\
H_{1}: \text { O modelo Slash Dobrado não pode ser reduzido ao modelo Normal Dobrado, }
\end{array}\right.
$$

ou, de forma equivalente,

$$
\left\{\begin{array}{l}
H_{0}: \quad \xi=0 \\
H_{1}: \quad \xi>0
\end{array}\right.
$$

Para testar estas hipótese pode-se utilizar o teste da razão de verossimilhanças. A função do teste é dada por:

$$
-2 \Delta(\hat{\gamma})=-2\left(\log \left(L_{0}(\hat{\gamma})\right)-\log \left(L_{1}(\hat{\gamma})\right)\right)
$$

em que $L_{0}(\hat{\gamma})$ é o máximo da função de verossimilhança sob $H_{0}$ e $L_{1}(\hat{\gamma})$ é o máximo da função de verossimilhança sob $H_{1}$. 
Sob $H_{0}$ tem-se que os dados seguem uma distribuição Normal Dobrada e os estimadores de máxima verossimilhança têm que ser obtidos como visto na Seção 3.1.1. Sob $H_{1}$ tem-se que os dados seguem uma distribuição Slash Dobrada e utilizam-se os estimadores de máxima verossimilhança vistos na Seção 3.1.2.

Em problemas regulares, sob $H_{0}$ a estatística $-2 \Delta(\hat{\gamma})$ segue uma distribuição Qui-quadrado com 1 grau de liberdade, ver Wilks [1962]. Entretanto, neste caso, temos um problema não regular e a validade do teste da razão de verossimilhanças precisa ser avaliada com maior cuidado. Uma forma de fazer isso é verificar se ocorre a propriedade de contiguidade nos moldes considerados em Sen \& Pedroso de Lima [2011]. No presente caso, a demonstração dessa propriedade torna-se muito complicada, e para avaliar a adequabilidade da distribuição qui-quadrado foi realizado um estudo de simulação, no qual serão geradas 1.000 réplicas de uma distribuição Normal Dobrada de tamanhos 100 e 1.000. Com essas réplicas serão encontrados os estimadores de máxima verossimilhança para os parâmetros sob os dois modelos, e então calculados os valores para a estatística de teste. Com estes valores, será avaliado graficamente se a distribuição Qui-quadrado (com 1 grau de liberdade) é adequada. Outro ponto importante é que, sob $H_{0}: \xi=0$, tem-se $q=\infty$, caracterizando um problema de fronteira.

Whelan \& Goldman [1999] analisaram por meio de estudos de simulação que em testes com hipóteses na fronteira do espaço paramétrico a distribuição da estatística do teste da razão de verossimilhanças, $-2 \Delta(\hat{\gamma})$, não seria uma distribuição qui-quadrado, mas sim uma mistura de quiquadrados. O teorema a seguir enuncia como seria a distribuição da estatística nesse caso.

Teorema 3.3.1. Suponha que a função de verossimilhança $L(\gamma)$ possui as três primeiras derivadas parciais em relação a todos os componentes de $\boldsymbol{\gamma}$, sendo que estas derivadas são limitadas por uma função cuja esperança exista para todo componente de $\boldsymbol{\gamma}$. Além disso, suponha que a matriz de Informação de Fisher é positiva definida. Então, o teste da razão de verossimilhanças para o teste $H_{0}: \boldsymbol{\gamma} \in \boldsymbol{\Gamma}_{0}$ versus $H_{1}: \boldsymbol{\gamma} \in \boldsymbol{\Gamma}_{1}$, tal que $\boldsymbol{\Gamma}_{0} \subset \boldsymbol{\Gamma}_{1}$, e apenas um dos componentes de $\boldsymbol{\gamma}$ está na fronteira do espaço paramétrico, sob $H_{0}$ temos que

$$
-2 \Delta(\hat{\gamma}) \sim 0,5 \chi_{r-1}^{2}+0,5 \chi_{r}^{2}
$$

em que $r$ é a diferença entre os parâmetros livres entre os modelos sob $H_{1}$ e $H_{0}$.

Demonstração: Ver Self \& Liang [1987]. 
Neste trabalho $r=1$, e assim, a distribuição assintótica seria

$$
-2 \Delta(\hat{\gamma}) \sim 0,5 \chi_{0}^{2}+0,5 \chi_{1}^{2}
$$

com $\chi_{0}^{2}$ uma distribuição degenerada no ponto 0 .

Ao realizar o estudo de simulação para verificar o erro tipo I cometido pelo teste foi considerado que sob $H_{0}$ a estatística do teste segue uma distribuição qui-quadrado com 1 grau de liberdade e, também, uma mistura de qui-quadrados, como mencionado anteriormente.

Na Tabela 3.5 encontram-se as quantidades de réplicas em que verificou-se o erro tipo I. Como referência, considerou-se uma probabilidade de erro tipo I igual a $5 \%$. Verifica-se que quando o tamanho amostral aumenta o percentual de erro se aproxima do valor esperado para a qui-quadrado, mas, para a mistura de qui-quadrados, esse valor é ultrapassado. Percebe-se ainda que conforme o percentual de censura aumenta o percentual de erro tipo I diminui, tanto para a distribuição qui-quadrado quanto para a mistura de qui-quadrados.

Nas Figuras 3.8, 3.9 e 3.10 encontram-se os gráficos dos quantis teóricos em função dos quantis empíricos. Na coluna à esquerda é considerada a distribuição qui-quadrado com 1 grau de liberdade, na coluna da direita é considerada a mistura de qui-quadrados com $r=1$. Pelos Gráficos verifica-se que conforme o percentual de censura aumenta as duas distribuições tendem a gerar gráficos com maior grau de inadequação. No entanto verifica-se que em ambos os casos com percentuais menores de censura os pontos ficam muito próximos das bandas de confiança.

Tabela 3.5: Erro tipo I cometido em 1.000 réplicas.

\begin{tabular}{ccccc}
\hline \hline \multirow{2}{*}{$\begin{array}{c}\text { Percentual } \\
\text { de censura }\end{array}$} & Qui-quadrado & Mistura & Qui-quadrado & Mistura \\
\hline $0 \%$ & $17(1,7 \%)$ & $45(4,5 \%)$ & $44(4,4 \%)$ & $74(7,4 \%)$ \\
$30 \%$ & $13(1,3 \%)$ & $34(3,4 \%)$ & $24(2,4 \%)$ & $63(6,3 \%)$ \\
$50 \%$ & $3(0,3 \%)$ & $14(1,4 \%)$ & $29(2,9 \%)$ & $63(6,3 \%)$ \\
\hline
\end{tabular}



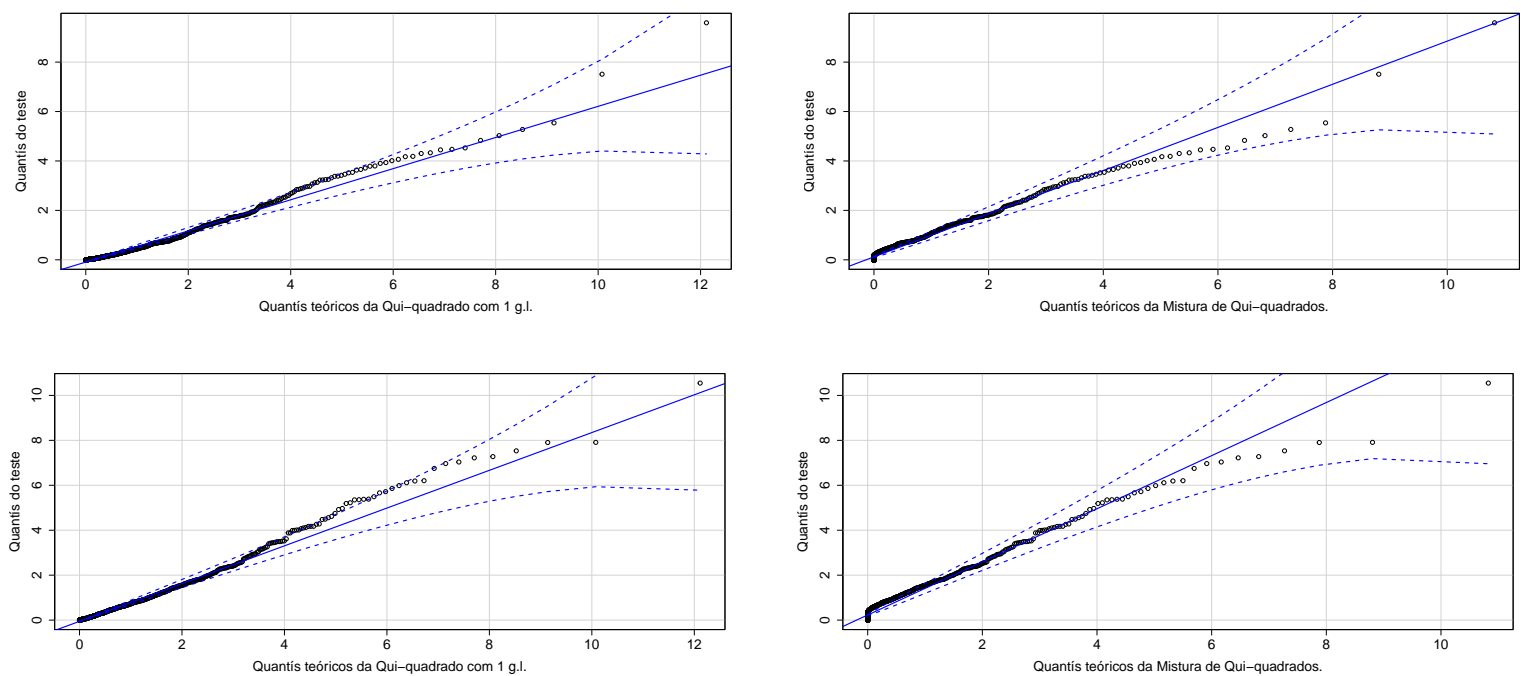

Figura 3.8: Gráficos dos quantis com 0\% de censura. Nas linhas $n=100$ e $n=1.000$, nas colunas quantis das distribuições qui-quadrado e Mistura de qui-quadrados
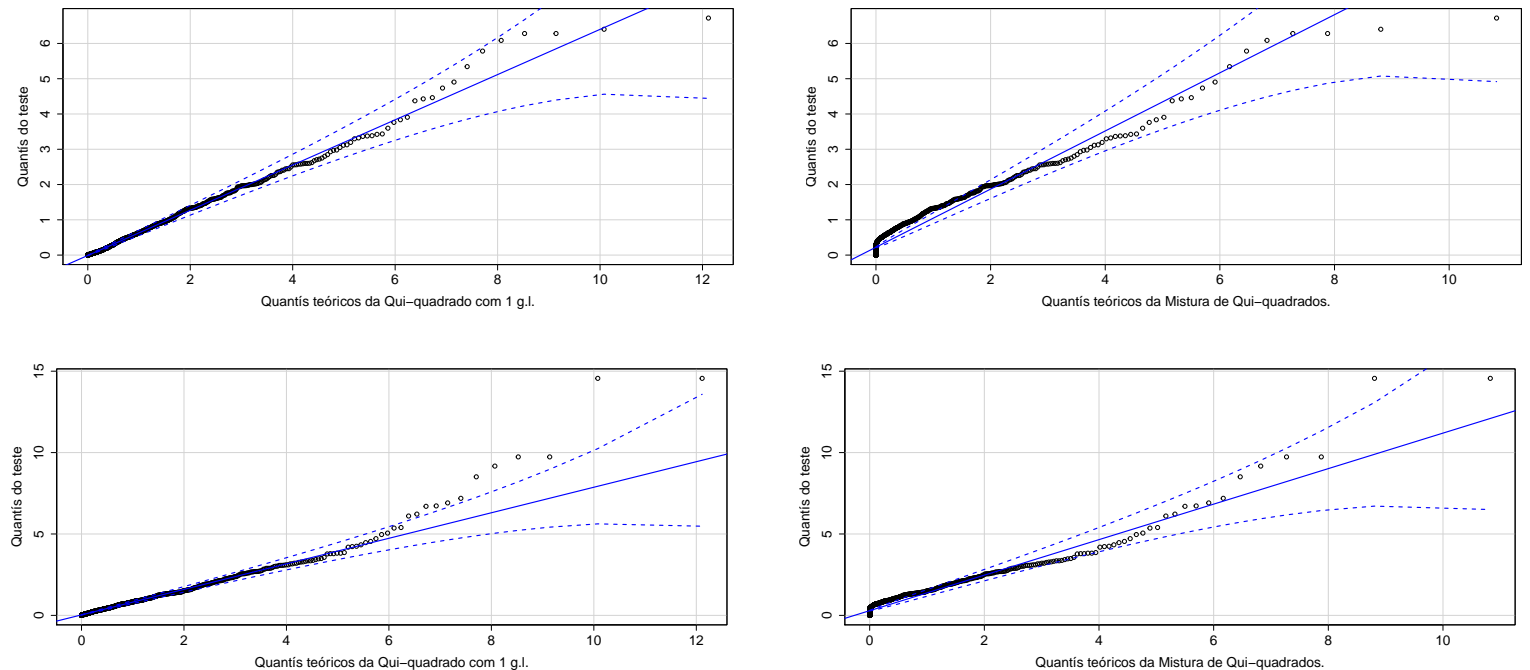

Figura 3.9: Gráficos dos quantis com 30\% de censura. Nas linhas $n=100$ e $n=1.000$, nas colunas quantis das distribuições qui-quadrado e Mistura de qui-quadrados 

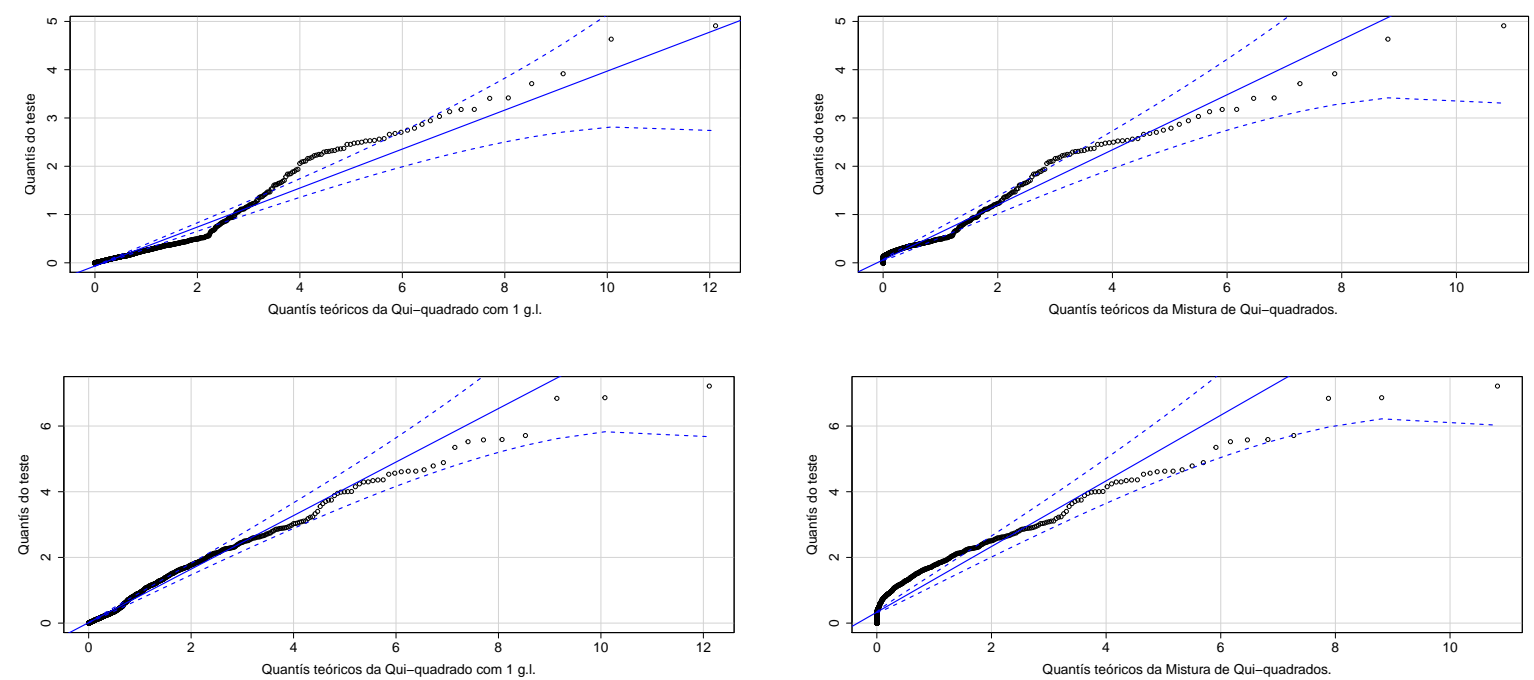

Figura 3.10: Gráficos dos quantis com $50 \%$ de censura. Nas linhas $n=100$ e $n=1.000$, nas colunas quantis das distribuições qui-quadrado e Mistura de qui-quadrados

É interessante notar que pelos valores mostrados na Tabela 3.5 a distribuição qui-quadrado aparenta ser mais adequada. Já pelos Gráficos 3.8, 3.9 e 3.10, a mistura de qui-quadrados apresenta maior adequabilidade. Portanto, é necessário mais estudos para verificar qual seria realmente a distribuição da estatística do teste da razão de verossimilhanças.

Considerando que sob $H_{0}$ a distribuição da estatística é qui-quadrado com 1 grau de liberdade, então sob $H_{1}$ a estatística $-2 \Delta(\hat{\gamma})$ segue uma distribuição qui-quadrado com 1 grau de liberdade e parâmetro de não centralidade $d$ dado por

$$
d=\xi^{2} \boldsymbol{I}(\xi)
$$

com $\boldsymbol{I}(\xi)$ o componente da matriz de informação observada correspondente ao parâmetro $\xi$.

Vale ressaltar que ao realizar um estudo de simulação para encontrar o valor do parâmetro de não centralidade deve-se considerar o método de máxima verossimilhança restrita. Dado o valor $\xi$, utilizado para gerar os dados, encontram-se os estimadores de máxima verossimilhança para os outros parâmetros. Com as estimativas desses parâmetros e o valor $\xi$, utilizando o componente da matriz de informação observada correspondente ao parâmetro $\xi$ obtém-se o valor $d$. Assim é possível analisar o poder do teste por meio de simulação. Neste trabalho não foi feito este estudo de simulação. 


\section{Capítulo 4}

\section{Heterogeneidade no modelo Slash}

\section{Dobrado}

Neste capítulo considera-se que os dados vêm de uma população heterogênea, em que covariáveis são definidas para controlar como as diferentes características dos indivíduos atuam na ocorrência dos eventos de interesse.

A heterogeneidade gera a necessidade de analisar os indivíduos separando por grupos. Os indivíduos de um determinado grupo possuem características semelhantes, mas distintas se comparadas com os indivíduos de outros grupos. Pressupõe-se que a sobrevida de indivíduos dentro de um dado grupo segue um mesmo padrão, então procura-se estimar a sobrevida para esse grupo.

\subsection{Inclusão de covariáveis}

Conforme dito anteriormente, no modelo Slash Dobrado pode-se atribuir covariáveis aos três parâmetros da distribuição. Neste trabalho será considerada a inclusão de variáveis independentes no parâmetro de posição $\mu$, e no parâmetro que controla o peso da cauda da distribuição $q$, o que permite determinar características que fazem um individuo ser de longo termo. Com isso, espera-se ser possível detectar grupos de indivíduos que apresentam o evento de interesse como o esperado e grupos de indivíduos que demoram mais para apresentar o evento. Ao incluir covariáveis quantitativas pode-se determinar como o aumento ou diminuição dos valores dessas covariáveis influenciam na probabilidade de um indivíduo ser de longo termo. Ao incluir variáveis independentes no parâmetro de posição, pode-se determinar características para estimar a sobrevida, tanto em dados de longo termo quanto naqueles que apresentam um padrão esperado. 
Considere uma população para a qual o modelo Slash Dobrado é adequado. Tomando-se uma amostra de tamanho $n$, considera-se $T_{i}, i=1, \ldots, n$, o tempo até o evento de interesse, tal que $T_{i} \sim$ Slash Dobrada $\left(\mu_{i}, \sigma, q_{i}\right), \mu_{i}=\exp \left(\boldsymbol{X}_{\mu i}^{T} \boldsymbol{\beta}_{\mu}\right)$, em que $\boldsymbol{\beta}_{\mu} \in \mathbb{R}^{p_{1}+1}$, e $\boldsymbol{X}_{\mu i}$ o vetor contendo um elemento 1 e $p_{1}$ covariáveis do $i$-ésimo indivíduo relacionadas ao parâmetro de posição, sendo ainda $q_{i}=\exp \left(\boldsymbol{X}_{q i}^{T} \boldsymbol{\beta}_{q}\right), \operatorname{com} \boldsymbol{\beta}_{q} \in \mathbb{R}^{p_{2}+1}$ e $\boldsymbol{X}_{q i}$ o vetor contendo um elemento 1 e as $p_{2}$ covariáveis do $i$-ésimo indivíduo relacionadas ao parâmetro que controla o peso da cauda da distribuição. A fim de observar o tempo até um evento de interesse define-se, como antes, $Z_{i}=\min \left\{T_{i}, C_{i}\right\}$ e $\delta_{i}=I\left(T_{i} \leq C_{i}\right), \operatorname{com} i=1, \ldots, n$.

Utilizando as funções densidade de probabilidade e sobrevivência vistas no capítulo anterior, mas agora incluindo covariáveis, chega-se à função de verossimilhança dada por

$$
\begin{aligned}
& L(\gamma)=\prod_{i=1}^{n}\left[f_{T_{i}}\left(z_{i}\right)\right]^{\delta_{i}}\left[S_{T_{i}}\left(z_{i}\right)\right]^{1-\delta_{i}} \\
& =\prod_{i=1}^{n}\left\{\int_{0}^{1} \frac{e^{\boldsymbol{X}_{q i}^{T} \boldsymbol{\beta}_{q}} u^{e^{\boldsymbol{X}_{q i}^{T} \boldsymbol{\beta}_{q}}}}{\sigma}\left[\frac{e^{-\frac{\left(u z_{i}-e^{\left.\boldsymbol{X}_{\mu i}^{T} \boldsymbol{\beta}_{\mu}\right)^{2}}\right.}{2 \sigma^{2}}}}{\sqrt{2 \pi}}+\frac{e^{-\frac{\left(u z_{i}+e^{\left.\boldsymbol{X}_{\mu i}^{T} \boldsymbol{\beta}_{\mu}\right)^{2}}\right.}{2 \sigma^{2}}}}{\sqrt{2 \pi}}\right] \mathrm{d} u\right\}^{\delta_{i}} \\
& \times \prod_{i=1}^{n}\left\{\int_{0}^{1} \frac{e^{\boldsymbol{X}_{q i}^{T} \boldsymbol{\beta}_{q}} u^{\boldsymbol{X}_{q i}^{T} \boldsymbol{\beta}_{q}}}{\sigma} \int_{z_{i}}^{\infty}\left[\frac{e^{-\frac{\left(u y-e^{\left.\boldsymbol{X}_{\mu i}^{T} \boldsymbol{\beta}_{\mu}\right)^{2}}\right.}{2 \sigma^{2}}}}{\sqrt{2 \pi}}+\frac{e^{-\frac{\left(u y+e^{\left.\boldsymbol{X}_{\mu i}^{T} \boldsymbol{\beta}_{\mu}\right)^{2}}\right.}{2 \sigma^{2}}}}{\sqrt{2 \pi}}\right] \mathrm{d} y \mathrm{~d} u\right\}^{1-\delta_{i}},
\end{aligned}
$$

em que $\boldsymbol{\gamma}=\left(\boldsymbol{\beta}_{\mu}^{T}, \sigma, \boldsymbol{\beta}_{q}^{T}\right)^{T}$.

Para determinar os estimadores de máxima verossimilhança dos parâmetros do modelo utiliza-se a log-verossimilhança dada por

$$
l(\boldsymbol{\gamma})=-n \log (\sigma)+\sum_{i=1}^{n} \boldsymbol{X}_{q i}^{T} \boldsymbol{\beta}_{q}+l_{1}+l_{2}
$$

em que

$$
l_{1}=\sum_{i=1}^{n} \delta_{i} \log \left(l_{1 i}\right), \quad l_{2}=\sum_{i=1}^{n}\left(1-\delta_{i}\right) \log \left(l_{2 i}\right),
$$

e 


$$
\begin{aligned}
& l_{1 i}=\int_{0}^{1} u^{e^{\boldsymbol{X}_{q i}^{T} \boldsymbol{\beta}_{q}}}\left[\frac{e^{-\frac{\left(u z_{i}-e^{\left.\boldsymbol{X}_{\mu i}^{T} \boldsymbol{\beta}_{\mu}\right)^{2}}\right.}{2 \sigma^{2}}}}{\sqrt{2 \pi}}+\frac{e^{-\frac{\left(u z_{i}+e^{\left.\boldsymbol{X}_{\mu i}^{T} \boldsymbol{\beta}_{\mu}\right)^{2}}\right.}{2 \sigma^{2}}}}{\sqrt{2 \pi}}\right] \mathrm{d} u \\
& =\int_{0}^{1} u^{e^{\boldsymbol{X}_{q i}^{T} \boldsymbol{\beta}_{q}}}\left[\phi\left(\frac{u z_{i}-e^{\boldsymbol{X}_{\mu i}^{T} \boldsymbol{\beta}_{\mu}}}{\sigma}\right)+\phi\left(\frac{u z_{i}+e^{\boldsymbol{X}_{\mu i}^{T} \boldsymbol{\beta}_{\mu}}}{\sigma}\right)\right] \mathrm{d} u, \\
& l_{2 i}=\int_{0}^{1} u^{e^{\boldsymbol{X}_{q i}^{T} \boldsymbol{\beta}_{q}}} \int_{z_{i}}^{\infty}\left[\frac{e^{-\frac{\left(u y-e^{\left.\boldsymbol{X}_{\mu i}^{T} \boldsymbol{\beta}_{\mu}\right)^{2}}\right.}{2 \sigma^{2}}}}{\sqrt{2 \pi}}+\frac{e^{-\frac{\left(u y+e \boldsymbol{X}_{\mu i}^{T} \boldsymbol{\beta}_{\mu}\right)^{2}}{2 \sigma^{2}}}}{\sqrt{2 \pi}}\right] \mathrm{d} y \mathrm{~d} u \\
& =\int_{0}^{1} u^{e^{\boldsymbol{X}_{q i}^{T} \boldsymbol{\beta}_{q}}-1} \sigma\left[S\left(\frac{u z_{i}-e^{\boldsymbol{X}_{\mu i}^{T} \boldsymbol{\beta}_{\mu}}}{\sigma}\right)+S\left(\frac{u z_{i}+e^{\boldsymbol{X}_{\mu i}^{T} \boldsymbol{\beta}_{\mu}}}{\sigma}\right)\right] \mathrm{d} u \text {. }
\end{aligned}
$$

Derivando o logaritmo da verossimilhança em relação à $\boldsymbol{\gamma}$ obtém-se

$$
\begin{aligned}
& \frac{\partial l(\gamma)}{\partial \boldsymbol{\beta}_{\mu}}=\frac{\partial l_{1}}{\partial \boldsymbol{\beta}_{\mu}}+\frac{\partial l_{2}}{\partial \boldsymbol{\beta}_{\mu}}, \\
& \frac{\partial l(\gamma)}{\partial \sigma}=-\frac{n}{\sigma}+\frac{\partial l_{1}}{\partial \sigma}+\frac{\partial l_{2}}{\partial \sigma}, \\
& \frac{\partial l(\gamma)}{\partial \boldsymbol{\beta}_{q}}=\sum_{i=1}^{n} \boldsymbol{X}_{q i}+\frac{\partial l_{1}}{\partial \boldsymbol{\beta}_{q}}+\frac{\partial l_{2}}{\partial \boldsymbol{\beta}_{q}}
\end{aligned}
$$

com

$$
\begin{aligned}
& \frac{\partial l_{1}}{\partial \boldsymbol{\beta}_{\mu}}=\sum_{i=1}^{n} \delta_{i} \frac{\int_{0}^{1} u^{e^{\boldsymbol{X}_{q i}^{T} \boldsymbol{\beta}_{q}}} e^{\boldsymbol{X}_{\mu i}^{T} \boldsymbol{\beta}_{\mu}} \boldsymbol{X}_{\mu i}\left[\phi\left(\frac{u z_{i}-e^{\boldsymbol{X}_{\mu i}^{T} \boldsymbol{\beta}_{\mu}}}{\sigma}\right)\left(\frac{z_{i} u-e^{\boldsymbol{X}_{\mu i}^{T} \boldsymbol{\beta}_{\mu}}}{\sigma^{2}}\right)-\phi\left(\frac{u z_{i}+e^{\boldsymbol{X}_{\mu i}^{T} \boldsymbol{\beta}_{\mu}}}{\sigma}\right)\left(\frac{z_{i} u+e^{\boldsymbol{X}_{\mu i}^{T} \boldsymbol{\beta}_{\mu}}}{\sigma^{2}}\right)\right] \mathrm{d} u}{l_{1 i}}, \\
& \frac{\partial l_{1}}{\partial \sigma}=\sum_{i=1}^{n} \delta_{i} \frac{\int_{0}^{1} u^{e^{\mathbf{X}_{q i}^{T} \boldsymbol{\beta}_{q}}}\left[\phi\left(\frac{u z_{i}-e^{\mathbf{X}_{\mu i}^{T} \boldsymbol{\beta}_{\mu}}}{\sigma}\right)\left(\frac{\left(z_{i} u-e^{\mathbf{X}_{\mu i}^{T} \boldsymbol{\beta}_{\mu}}\right)^{2}}{\sigma^{3}}\right)+\phi\left(\frac{u z_{i}+e^{\mathbf{X}_{\mu i}^{T} \boldsymbol{\beta}_{\mu}}}{\sigma}\right)\left(\frac{\left(z_{i} u+e^{\mathbf{X}_{\mu i}^{T} \boldsymbol{\beta}_{\mu}}\right)^{2}}{\sigma^{3}}\right)\right] \mathrm{d} u}{l_{1 i}}, \\
& \frac{\partial l_{1}}{\partial \boldsymbol{\beta}_{q}}=\sum_{i=1}^{n} \delta_{i} \frac{\int_{0}^{1} u^{e^{\boldsymbol{X}_{q i}^{T} \boldsymbol{\beta}_{q}}} e^{\boldsymbol{X}_{q i}^{T} \boldsymbol{\beta}_{q}} \boldsymbol{X}_{q i} \log (u)\left[\phi\left(\frac{u z_{i}-e^{\boldsymbol{X}_{\mu i}^{T} \boldsymbol{\beta}_{\mu}}}{\sigma}\right)+\phi\left(\frac{u z_{i}+e^{\boldsymbol{X}_{\mu i}^{T} \boldsymbol{\beta}_{\mu}}}{\sigma}\right)\right] \mathrm{d} u}{l_{1 i}}, \\
& \frac{\partial l_{2}}{\partial \boldsymbol{\beta}_{\mu}}=\sum_{i=1}^{n}\left(1-\delta_{i}\right) \frac{\int_{0}^{1} u^{e^{\boldsymbol{X}_{q i}^{T} \boldsymbol{\beta}_{q}}-1} e^{\boldsymbol{X}_{\mu i}^{T} \boldsymbol{\beta}_{\mu}} \boldsymbol{X}_{\mu i}\left[\phi\left(\frac{u z_{i}-e^{\boldsymbol{X}_{\mu i}^{T} \boldsymbol{\beta}_{\mu}}}{\sigma}\right)-\phi\left(\frac{u z_{i}+e^{\boldsymbol{X}_{\mu i}^{T} \boldsymbol{\beta}_{\mu}}}{\sigma}\right)\right] \mathrm{d} u}{l_{2 i}}, \\
& \frac{\partial l_{2}}{\partial \sigma}=\sum_{i=1}^{n}\left(1-\delta_{i}\right) \frac{\int_{0}^{1} u^{e^{\boldsymbol{X}_{q i}^{T} \boldsymbol{\beta}_{q}}-1} / 2\left[S_{\Gamma}\left(\frac{\left(u z_{i}-e^{\boldsymbol{X}_{\mu i}^{T} \boldsymbol{\beta}_{\mu}}\right)^{2}}{2 \sigma^{2}}, \frac{3}{2}, 1\right)+S_{\Gamma}\left(\frac{\left(u z_{i}+e^{\boldsymbol{X}_{\mu i}^{T} \boldsymbol{\beta}_{\mu}}\right)^{2}}{2 \sigma^{2}}, \frac{3}{2}, 1\right)\right] \mathrm{d} u}{l_{2 i}},
\end{aligned}
$$


$\frac{\partial l_{2}}{\partial \boldsymbol{\beta}_{q}}=\sum_{i=1}^{n}\left(1-\delta_{i}\right) \frac{\int_{0}^{1} u^{e^{\boldsymbol{X}_{q i}^{T} \boldsymbol{\beta}_{q}}-1} e^{\boldsymbol{X}_{q i}^{T} \boldsymbol{\beta}_{q}} \boldsymbol{X}_{q i} \log (u) \sigma\left[S\left(\frac{u z_{i}-e^{\boldsymbol{X}_{\mu i}^{T} \boldsymbol{\beta}_{\mu}}}{\sigma}\right)+S\left(\frac{u z_{i}+e^{\boldsymbol{X}_{\mu i}^{T} \boldsymbol{\beta}_{\mu}}}{\sigma}\right)\right] \mathrm{d} u}{l_{2 i}}$.

Para as expressões das segundas derivadas incluindo as transformações propostas para simplificar as rotinas computacionais, veja a Seção A.3.

As expressões para a verossimilhança e o vetor escore são semelhantes àquelas obtidas para o caso sem covariáveis. Novamente elas são expressas em termos da função densidade de probabilidade e distribuição acumulada da Normal padrão por motivos computacionais.

\subsection{Teste de Wald}

Nesta Seção são discutidos aspectos relacionados ao procedimento de verificação da magnitude dos efeitos de covariáveis, principalmente a influência delas no parâmetro $q_{i}$ que controla o aparecimento de tempos de maior magnitude.

Dada a parametrização adotada na seção anterior, considera-se as hipóteses

$$
\left\{\begin{array}{ll}
H_{0}: & \beta_{q j}=0 ; \\
H_{1}: & \beta_{q j} \neq 0
\end{array} \quad j=0,1, \ldots, p_{2} .\right.
$$

Para verificar se $H_{0}$ é rejeitada, será utilizado o teste de Wald por sua simplicidade. Considerando o fato dos parâmetros serem ortogonais, e consequentemente a matriz de informação bloco diagonal, sob $H_{0}$, a estatística do teste é dada por

$$
W=\frac{\hat{\beta}_{q j}^{2}}{\widehat{\operatorname{Var}\left(\hat{\beta}_{q j}\right)}},
$$

Considerando os resultados das simulações apresentadas no capítulo anterior conjectura-se aqui que o estimador de máxima verossimilhança para $\beta_{q j}$ é assintoticamente Normal e, assim, sob $H_{0}$ a estatística $W$ tem distribuição que pode ser aproximada por uma distribuição de qui-quadrado com 1 graus de liberdade, ver Pawitan [2001].

No contexto da comparação de dois grupos, supondo $X_{q i}$ uma covariável dicotômica, tem-se que a não rejeição de $H_{0}$ implica que os dois grupos considerados não possuem diferentes probabilidades de ocorrência de dados de longo termo, pois as caudas das distribuições para os dois grupos teriam o mesmo peso. 
O teste de Wald pode também ser realizado para avaliar o efeito de covariáveis incluídas no parâmetro $\mu$. Neste caso a estatística do teste seria dada por

$$
W=\frac{\hat{\beta}_{\mu j}^{2}}{\operatorname{Var}\left(\hat{\beta}_{\mu j}\right)}, j=0,1, \ldots, p_{1},
$$

assim como o caso do teste para o parâmetro $\beta_{q j}$, a estatística $W$ pode ser aproximada por uma distribuição qui-quadrado com 1 grau de liberdade. 


\subsection{Estudo de simulação}

Nesta seção procura-se avaliar o comportamento dos estimadores de máxima verossimilhança $\boldsymbol{\beta}_{\mu}$ e $\boldsymbol{\beta}_{q}$ em amostras de diferentes tamanhos, sujeitos à censura na proporção de $25 \%$. Foram analisadas combinações diversas de percentuais de censura, indo de $0 \%$ até $50 \%$, considerando censuras aleatória e tipo I. No entanto, aqui só foram apresentados os resultados com $25 \%$ de censura.

O estudo de simulação foi considerado assumindo para o parâmetro $\mu$ duas covariáveis, uma dicotômica e outra quantitativa, enquanto que apenas uma covariável dicotômica foi incluída no parâmetro q. Especificamente,

$$
\mu_{i}=\exp \left(\beta_{\mu 0}+\beta_{\mu 1} x_{\mu 1 i}+\beta_{\mu 2} x_{\mu 2 i}\right) \text { e } q_{i}=\exp \left(\beta_{q 0}+\beta_{q 1} x_{q 1 i}\right) .
$$

De forma análoga ao capítulo anterior, foram geradas amostras com diferentes proporções de censura à direita de tamanho 100, 500 e 1.000. Entretanto, devido à complexidade do algoritmo e a quantidade de parâmetros a serem estimados, nesta seção foi apresentado um número menor de cenários analisados. Apesar de não mostrado aqui, uma avaliação de outros cenários com outras proporções de censura forneceu padrões semelhantes ao observado no Capítulo 3.

Para a geração dos dados no caso com covariáveis o procedimento aqui também é mais complexo do que no caso sem covariáveis. Inicialmente foram gerados valores para as covariáveis. Para as dicotômicas, foi utilizada uma distribuição de Bernoulli com proporção de sucesso igual a meio. Para a geração da covariável quantitativa foi utilizada a distribuição Normal padrão. Dados os valores das covariáveis e os valores dos parâmetros pré-fixados, $\boldsymbol{\beta}_{\mu}=(1,-3,2)^{T}$ e $\boldsymbol{\beta}_{q}=(1,-2)^{T}$, foram gerados os tempos de falhas, $T_{i}$ com distribuição Slash Dobrada $\left(\mu_{i}, \sigma, q_{i}\right), i=1, \ldots, n$. Em seguida, foi determinado se os tempos seriam falha ou censura. Para censuras aleatórias foram gerados valores de uma Bernoulli com o percentual de censura de interesse. Para aqueles indivíduos a serem censurados, foi gerado aleatoriamente um tempo de uma censura, a partir de uma distribuição Uniforme Contínua no intervalo zero ao mínimo entre $\tau$ e o valor obtido como sendo o tempo de falha. Em seguida, valores maiores que $\tau$ foram substituídos por $\tau$. Dessa forma atingiu-se, em média, o percentual de censura pré-especificado.

No caso sem covariáveis do Capítulo 3, tendo os valores verdadeiros dos parâmetros é possível verificar qual seria o valor de $\tau$ que deixaria o percentual de censura do tipo I de interesse. No 
entanto, neste capítulo, a inclusão de covariáveis interfere com a distribuição dos dados. Foram gerados os valores de cada réplica, e em cada réplica foi verificado um valor que deixaria o percentual de censura de interesse à direita desse. Em seguida foi obtido um $\tau$ médio igual ao percentual de censura tipo I de interesse.

Na Tabela 4.1 são apresentadas informações resultantes das estimativas de $\boldsymbol{\beta}_{\mu}$. Assim como no caso sem covariáveis, verifica-se superestimação do parâmetro $\mu$. Observa-se ainda que conforme o tamanho amostral aumenta, os erros padrão vão diminuindo. Esse comportamento fica mais evidente conforme o percentual de censura aumenta (resultado não mostrado aqui). Nota-se ainda que o vício e erro quadrático médio não são grandes, mesmo com o problema de superestimação.

Tabela 4.1: Estimativas para os parâmetros da distribuição $\boldsymbol{\beta}_{\mu}=(1,-3,2)$ com $25 \%$ de censura, em 100 réplicas de tamanho igual a 100, 500 e 1.000.

\begin{tabular}{|c|c|c|c|c|c|c|c|c|c|c|}
\hline \multirow{2}{*}{$\begin{array}{l}\text { Tipo de } \\
\text { Censura }\end{array}$} & & \multicolumn{3}{|c|}{$n=100$} & \multicolumn{3}{|c|}{$n=500$} & \multicolumn{3}{|c|}{$n=1.000$} \\
\hline & & $\hat{\beta}_{\mu 0}$ & $\hat{\beta}_{\mu 1}$ & $\hat{\beta}_{\mu 2}$ & $\hat{\beta}_{\mu 0}$ & $\hat{\beta}_{\mu 1}$ & $\hat{\beta}_{\mu 2}$ & $\hat{\beta}_{\mu 0}$ & $\hat{\beta}_{\mu 1}$ & $\hat{\beta}_{\mu 2}$ \\
\hline $0 \%$ & Média & 1,30 & $-2,49$ & 2,30 & 1,43 & $-2,27$ & 2,53 & 1,79 & $-2,14$ & 2,75 \\
\hline aleatória & Erro padrão & 0,81 & 1,02 & 0,80 & 0,63 & 0,75 & 0,49 & 0,56 & 0,40 & 0,32 \\
\hline$+25 \%$ & Vício & 0,30 & 0,51 & 0,30 & 0,43 & 0,73 & 0,53 & 0,79 & 0,86 & 0,75 \\
\hline tipo I & EQM & 0,74 & 1,30 & 0,73 & 0,58 & 1,09 & 0,52 & 0,94 & 0,90 & 0,66 \\
\hline
\end{tabular}

Na Tabela 4.2 encontram-se os resultados associados às estimativas para $\boldsymbol{\beta}_{q}$. Aqui também observa-se que o procedimento estima adequadamente o parâmetro $q$. Nota-se também que o erro padrão diminui conforme o tamanho amostral aumenta. Verifica-se ainda que o vício e o erro quadrático médio diminuem com o aumento amostral.

Tabela 4.2: Estimativas para os parâmetros da distribuição $\boldsymbol{\beta}_{q}=(1,-2)$ com $25 \%$ de censura, em 100 réplicas de tamanho igual a 100, 500 e 1.000.

\begin{tabular}{clrrrrrr}
\hline \hline \multirow{2}{*}{$\begin{array}{c}\text { Tipo de } \\
\text { Censura }\end{array}$} & & \multicolumn{2}{c}{$n=100$} & \multicolumn{2}{c}{$n=500$} & \multicolumn{2}{c}{$n=1.000$} \\
$0 \%$ & Média & 0,90 & $-1,99$ & 1,00 & $-2,06$ & 1,01 & $-2,03$ \\
\hline \multirow{2}{*}{ aleatória } & Erro padrão & 0,81 & 0,91 & 0,57 & 0,53 & 0,22 & 0,20 \\
$+25 \%$ & Vício & $-0,10$ & 0,01 & $<0,01$ & $-0,06$ & 0,01 & $-0,03$ \\
tipo I & EQM & 0,65 & 0,82 & 0,33 & 0,28 & 0,05 & 0,04 \\
\hline \hline
\end{tabular}

Para a estimação do parâmetro $\sigma$ observa-se o mesmo padrão tanto no caso com covariáveis 
quanto sem covariáveis, em que ocorre a superestimação conforme o percentual de censura aumenta e diminui o erro padrão conforme o tamanho amostral aumenta.
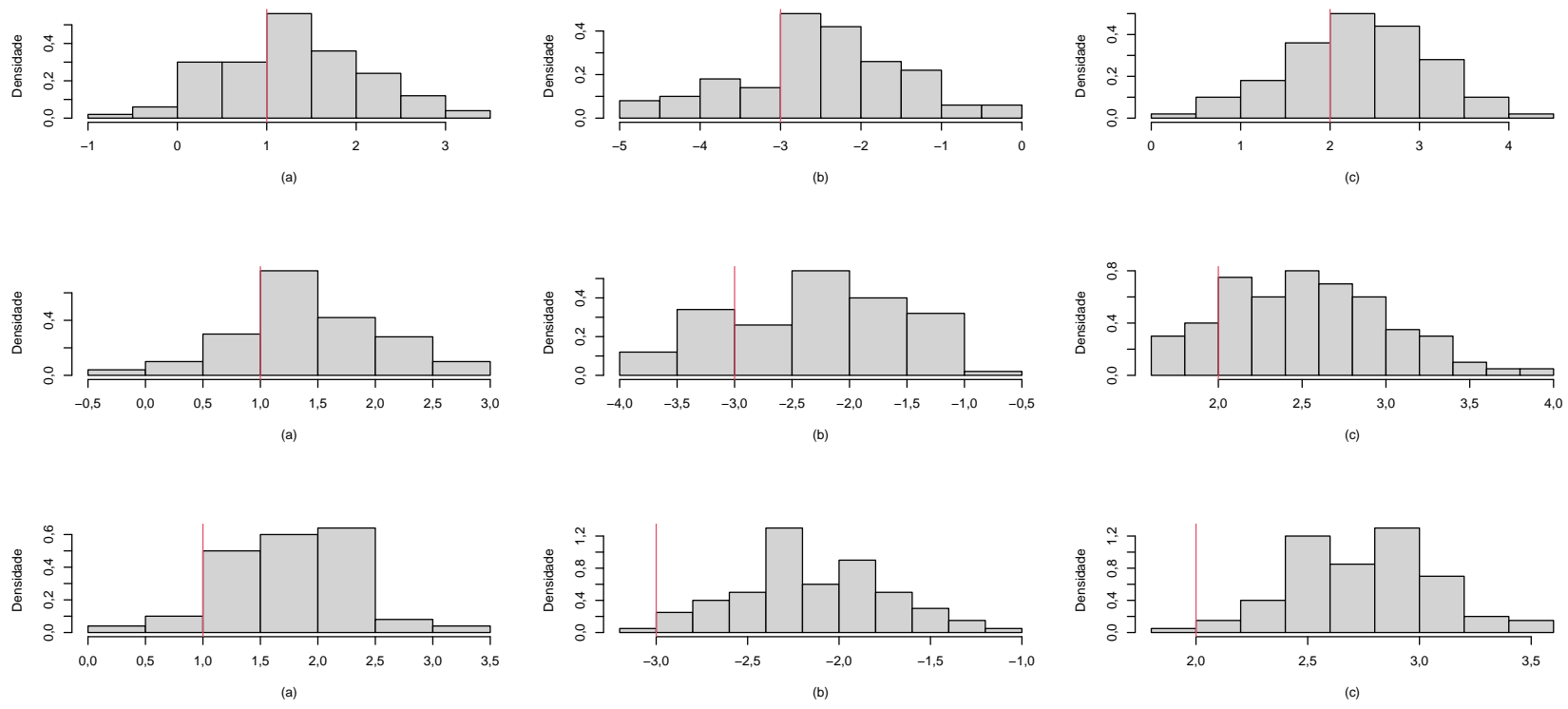

Figura 4.1: Histogramas das estimativas dos parâmetros $\boldsymbol{\beta}_{\mu}=(1,-3,2)$. Nas colunas tem-se os parâmetros, (a) $\beta_{\mu 0}$, (b) $\beta_{\mu 1}$ e (c) $\beta_{\mu 2}$, com 0\% de censura aleatória e 25\% tipo I em 100 réplicas. Nas linhas tem-se os tamanhos amostrais, 100, 500 e 1.000 .
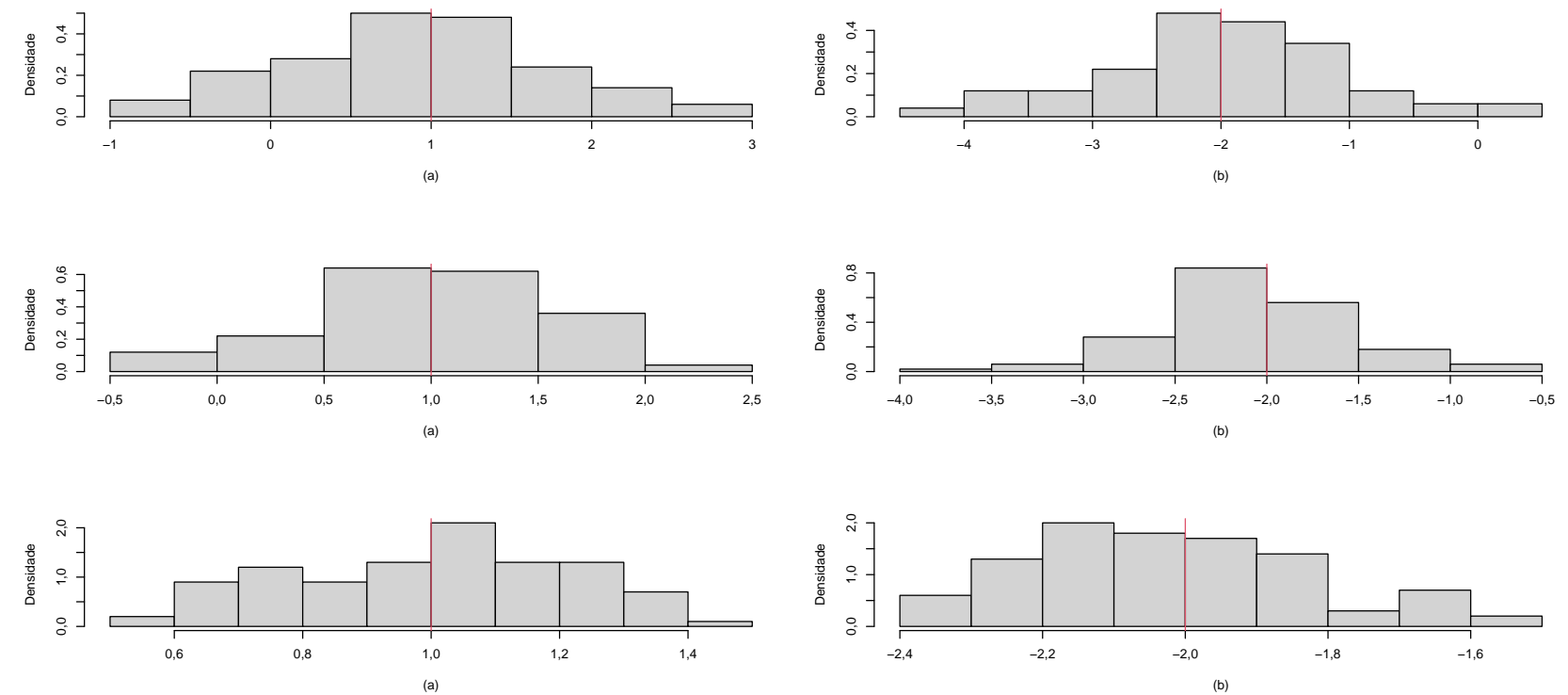

Figura 4.2: Histogramas das estimativas dos parâmetros $\boldsymbol{\beta}_{q}=(1,-2)$. Nas colunas tem-se os parâmetros, (a) $\beta_{q 0}$ e (b) $\beta_{q 1}$, com $0 \%$ de censura aleatória e $25 \%$ tipo I em 100 réplicas. Nas linhas tem-se os tamanhos amostrais, 100, 500 e 1.000 .

Os Histogramas 4.1 e 4.2 apresentam um padrão parecido com a distribuição Normal, o que sugere ser válida a distribuição qui-quadrado para a estatística de Wald.

Devido ao mesmo padrão encontrado nos outros cenários não foram incluídos neste trabalho 
outras tabelas e gráficos, pois estes não trariam novas informações se verificar o que já foi exposto nos casos com e sem covariáveis. Verificou-se que aumentando o percentual de censura o procedimento tende a superestimar os parâmetros $\mu$ e $\sigma$, mas em relação ao parâmetro $q$ o procedimento é bem estável, independente do percentual de censura. 


\section{Capítulo 5}

\section{Aplicação aos dados do projeto ICESP}

Neste capítulo considera-se o modelo Slash Dobrado para avaliar a presença de pacientes de longo termo nos dados do projeto ICESP, descrito na Seção 1.1. Recorde que, nesse estudo, pacientes oncológicos com a doença em estado avançado tem o início de seu acompanhamento definido como o momento de internação na UTI do ICESP e do Hospital de Barretos. O evento de interesse é a morte do paciente e, alguns pacientes sobrevivem mais do que esperado, sendo que vários deles encontravam-se vivos ao final do estudo (caracterizando assim dados com censura tipo I).

\subsection{Adequabilidade do modelo}

Inicialmente, admitindo-se que o modelo Normal Dobrado é adequado aos dados, testa-se a existência de dados de longo termo, isto é, se o modelo deveria ser, na verdade, o modelo Slash Dobrado. Neste momento não foram incluídas covariáveis. Assim foi realizado o teste da razão de verossimilhanças proposto na seção 3.3, com as seguintes hipóteses

$$
\begin{cases}H_{0}: & \text { Os dados seguem distribuição Normal Dobrada } \\ H_{1}: & \text { Os dados seguem distribuição Slash Dobrada }\end{cases}
$$

ou de forma equivalente

$$
\begin{cases}H_{0}: & \xi=0 \\ H_{1}: & \xi>0\end{cases}
$$

$\operatorname{com} \xi=1 / q$.

Para a verificação dessas hipóteses, considera-se o teste da razão de verossimilhanças discutido na Seção 3.3. Supondo que a estatística do teste segue uma distribuição de qui-quadrado com 1 
grau de liberdade, observa-se $\mathrm{p}<0,001$. O mesmo resultado foi obtido se for considerado o problema de fronteira e se for utilizada a mistura de qui-quadrados como a distribuição da estatística do teste. Consequentemente, rejeita-se a hipótese $H_{0}$ em favor do uso do modelo Slash Dobrado para os dados, o que em termos práticos sugere a presença de pacientes de longo termo.

\subsection{Inclusão de covariáveis}

Os dados incluem um grande número de variáveis que caracterizam os pacientes. Para exemplificar o uso do modelo Slash Dobrado com covariáveis, após uma análise descritiva inicial, baseada no estimador de Kaplan-Meier, decidiu-se considerar as características demográficas sexo e idade, e o status de cirurgia. A escolha dessas variáveis não foi feita seguindo critérios clínicos, mas por conveniência com relação à facilidade de implementação nos programas desenvolvidos, a fim de possibilitar avaliar o uso de variáveis de natureza qualitativa e quantitativa e, também para evitar ter que lidar com dados omissos. As variáveis dicotômicas foram definidas da seguinte forma

$$
\begin{gathered}
X_{i}= \begin{cases}0, & \text { se o i-ésimo paciente passou por cirurgia agendada; } \\
1, & \text { se o i-ésimo paciente não passou por cirurgia agendada. }\end{cases} \\
\operatorname{Sexo}_{i}= \begin{cases}0, & \text { se o i-ésimo paciente é do sexo feminino; } \\
1, & \text { se o i-ésimo paciente é do sexo masculino. }\end{cases}
\end{gathered}
$$

Idade $_{i}$ : idade em anos do paciente i.

Assumindo que um paciente submetido a cirurgia agendada teria um prognóstico melhor, essa informação foi incluída no parâmetro que controla o peso da cauda da distribuição Slash Dobrada, a fim de verificar se ela estaria influenciando a probabilidade do paciente ser de longo termo, isto é, de apresentar tempo de sobrevida maior que o esperado, relativamente aos outros pacientes. Considerou-se portanto a parametrização

$$
q_{i}=\exp \left\{\beta_{q 0}+\beta_{q 1} X_{i}\right\}, i=1, \ldots, 802,
$$

com $X_{i}$ definido como antes, $\beta_{q 0}$ seria o parâmetro associado com o peso da cauda para um paciente 
que se submeteu a uma cirurgia agendada e $\beta_{q 1}$ seria o parâmetro representando a mudança no peso da cauda (atuando multiplicativamente em $q_{i}$ ) para um paciente submetido a uma cirurgia de emergência.

As estimativas para o parâmetro que controla o peso da cauda da distribuição irão determinar se ao menos um dos dois grupos possui maior probabilidade de ter indivíduos de longo termo. Quanto menor forem os valores $\exp \left\{\hat{\beta}_{q 0}\right\}$ e $\exp \left\{\hat{\beta}_{q 0}+\hat{\beta}_{q 1}\right\}$ maior será a probabilidade da existência de dados de longo termo no grupo de cirurgia agendada e não agendada, respectivamente. As covariáveis Sexo e Idade foram incluídas no parâmetro de posição $\mu$, considerando a seguinte parametrização

$$
\mu_{i}=\exp \left\{\beta_{\mu 0}+\beta_{\mu 1} \operatorname{Sexo}_{i}+\beta_{\mu 2} \operatorname{Idade}_{i}\right\}, i=1, \ldots, 802 .
$$

com $\operatorname{Sexo}_{i}$ e Idade $i$ definidas como antes, $\beta_{\mu 1}$ representando o efeito de sexo e $\beta_{\mu 2}$ o efeito de idade. Neste caso $\beta_{\mu 0}$ não tem uma interpretação útil, o que pode ser feito centralizando-se a covariável Idade $_{i}$

A interpretação dos coeficientes será feita da seguinte forma. Para valores positivos (negativos) de $\beta_{\mu 1}$ a sobrevida aumenta (diminui) para os pacientes do sexo masculino, em relação aos pacientes do sexo feminino. Para a covariável Idade, se $\beta_{\mu 2}$ for positivo (negativo) a sobrevida aumenta (diminui) com o aumento da idade dos pacientes.

\subsection{Modelo estimado}

Na Tabela 5.1 encontram-se as estimativas de máxima verossimilhança dos parâmetros do modelo Slash Dobrado ajustado aos dados do projeto ICESP. Pode-se concluir pelas estimativas pontuais que a sobrevida dos pacientes do sexo feminino é maior que àquela dos pacientes do sexo masculino. Verifica-se também que a sobrevida dos pacientes diminui conforme a idade aumenta. Nota-se, ainda, que a probabilidade dos pacientes que passaram por cirurgia agendada serem de longo termo é maior que àquela de pacientes que passaram por cirurgia emergencial (não agendada).

Tabela 5.1: Estimativas de máxima verossimilhança para os parâmetros do modelo Slash Dobrado aplicado ao projeto ICESP.

\begin{tabular}{lrrrrrr}
\hline \hline & $\hat{\beta}_{\mu 0}$ & $\hat{\beta}_{\mu 1}$ & $\hat{\beta}_{\mu 2}$ & $\hat{\sigma}$ & $\hat{\beta}_{q 0}$ & $\hat{\beta}_{q 1}$ \\
\hline Estimativa & $-11,99$ & $-0,68$ & $-0,01$ & 10,80 & $-2,06$ & 1,12 \\
Erro padrão & 1,83 & 0,95 & 0,04 & 1,19 & 0,08 & 0,09 \\
\hline \hline
\end{tabular}


Ao analisar as estimativas pontuais dos parâmetros originais, conclui-se que ao considerar os grupos das mulheres, ou dos homens, com menor, ou maior, idade, obtém-se que a estimativa de máxima verossimilhança de $\mu$ é próxima de zero. Este achado sugere que um grande número de pacientes tem tempos de sobrevida pequenos relativamente à duração do experimento. De fato, conforme pode ser verificado na Tabela 1.3, o tempo mediano para os pacientes que foram a óbito é de 46 dias e o primeiro quartil é de 13 dias.

Ao obter as estimativas de máxima verossimilhança pontuais de $q$ para os grupos de pacientes que passaram ou não por cirurgia agendada encontra-se valores pequenos, sendo 0,13 e 0,39 respectivamente. Assim pode-se concluir que os dois grupos de pacientes tem grandes probabilidades de apresentar pacientes de longo termo, uma vez que ambos os valores são menores do que 1 .

Ao comparar as estimativas pontuais de máxima verossimilhança $\mu=0$ e $\sigma=10,80$ comprova-se uma grande assimetria, como foi visto anteriormente na Seção 2.1.1.

\subsection{Significância das covariáveis}

Para verificar se as conclusões anteriores podem ser extrapoladas para a população geral de pacientes da UTI do ICESP e do Hospital de Barretos, considera-se o teste de Wald discutido na Seção 4.2. O teste foi aplicado individualmente a cada parâmetro, considerando as seguintes hipóteses.

$H_{01} \begin{cases}H_{0}: & \beta_{\mu 1}=0 \text { (não há efeito de sexo no tempo de sobrevida); } \\ H_{1}: & \beta_{\mu 1} \neq 0 \text { (existe efeito de sexo no tempo de sobrevida). }\end{cases}$
$H_{02} \begin{cases}H_{0}: & \beta_{\mu 2}=0 \text { (o tempo de sobrevida não muda com a idade); } \\ H_{1}: & \beta_{\mu 2} \neq 0 \text { (existe efeito da idade na sobrevida). }\end{cases}$
$H_{03} \begin{cases}H_{0}: & \beta_{q 1}=0 \text { (cirurgia agendada não influencia na probabilidade de longo termo); } \\ H_{1}: & \beta_{q 1} \neq 0 \text { (cirurgia agendada influencia na probabilidade de longo termo). }\end{cases}$

Os resultados do teste estão Tabela 5.2. Conclui-se que as covariáveis Sexo e Idade não tem efeitos significativos, isto é, o parâmetro $\mu$ não é influenciado por essas covariáveis. Já a covariável dicotômica $X_{i}$, que indica se os pacientes passaram por cirurgia agendada, é altamente significativa. 
Tabela 5.2: Valor $p$ do teste de Wald para as covariáveis do modelo Slash Dobrado.

\begin{tabular}{lccccc}
\hline \hline & $\beta_{\mu 0}$ & $\beta_{\mu 1}$ & $\beta_{\mu 2}$ & $\beta_{q 0}$ & $\beta_{q 1}$ \\
\hline Valor da estatística & 42,928 & 0,512 & 0,063 & 663,063 & 154,864 \\
Valor $p$ & $<0,001$ & 0,474 & 0,803 & $<0,001$ & $<0,001$ \\
\hline \hline
\end{tabular}

Considerando as estimativas, $\hat{\mu}=0, \hat{q}=0,13$ para pacientes com cirurgia agendada e $\hat{q}=$ 0,39 para pacientes com cirurgia não agendada, as curvas de sobrevivência para as distribuições Slash Dobrado correspondentes são dadas na Figura 5.1. Verifica-se que no caso dos pacientes que passaram por cirurgia agendada a curva decai mais lentamente.

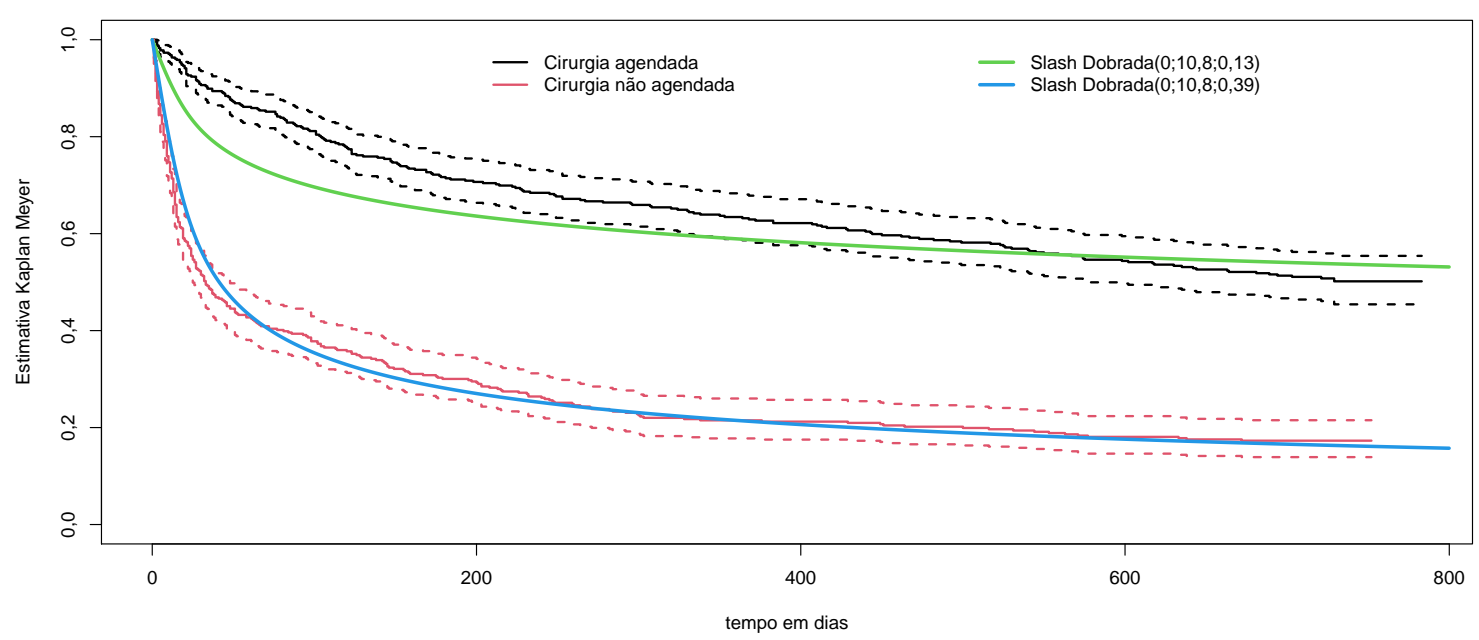

Figura 5.1: Estimativas das curvas de sobrevivência para distribuição Slash Dobrado considerando os valores estimados.

Considerando estas funções de sobrevivência estimadas, pode-se concluir que as probabilidades de um paciente sobreviver mais de 783 dias, valor máximo de acompanhamento observado nos dados, seriam respectivamente 0,53 e 0,16 , para os pacientes que passaram por cirurgia agendada ou não agendada. O tempo mediano de sobrevivência para pacientes nos dois grupos é, respectivamente, 1.275,4 e 41,2 dias. Ao incluir outras covariáveis pode-se melhorar a estimação da curva de sobrevivência. Verifica-se ainda que as curvas estimadas pelo modelo Slash Dobrado estão próximas das curvas estimadas de Kaplan-Meier, no entanto para o grupo de pacientes que passaram por cirurgia agendada a curva estimada pelo modelo Slash Dobrado decai mais lentamente, ficando mais próxima do limite superior da banda de confiança. 


\subsection{Resíduos Cox-Snell}

Considerando o modelo Slash Dobrado visto nos Capítulos 3 e 4 foi realizada uma comparação do modelo proposto, Slash Dobrado, e modelos comumente utilizados na literatura. Em dados de longo termo é comum utilizar modelos de mistura, nos quais é considerado que os tempos de falha seguem uma distribuição Weibull, Gama, Log Logístico, Log Normal, dentre outras. Uma característica interessante que vale ressaltar é que os modelos de mistura possuem função de sobrevivência imprópria, o que não ocorre no modelo Slash Dobrado.

Com base neste modelo e nos mencionados no início do capítulo foram analisados os resíduos de Cox-Snell. Os resíduos de Cox-Snell não identificam as causas de possíveis problemas nos modelos, mas indicam quando um modelo não está adequado, ver Cox \& Snell [1968]. Assim pela Figura 5.2 pode-se verificar que o modelo Slash Dobrado está um pouco mais próximo da reta de $45^{\circ}$, em vários instantes, o que sugere que este modelo está melhor ajustado que os outros.

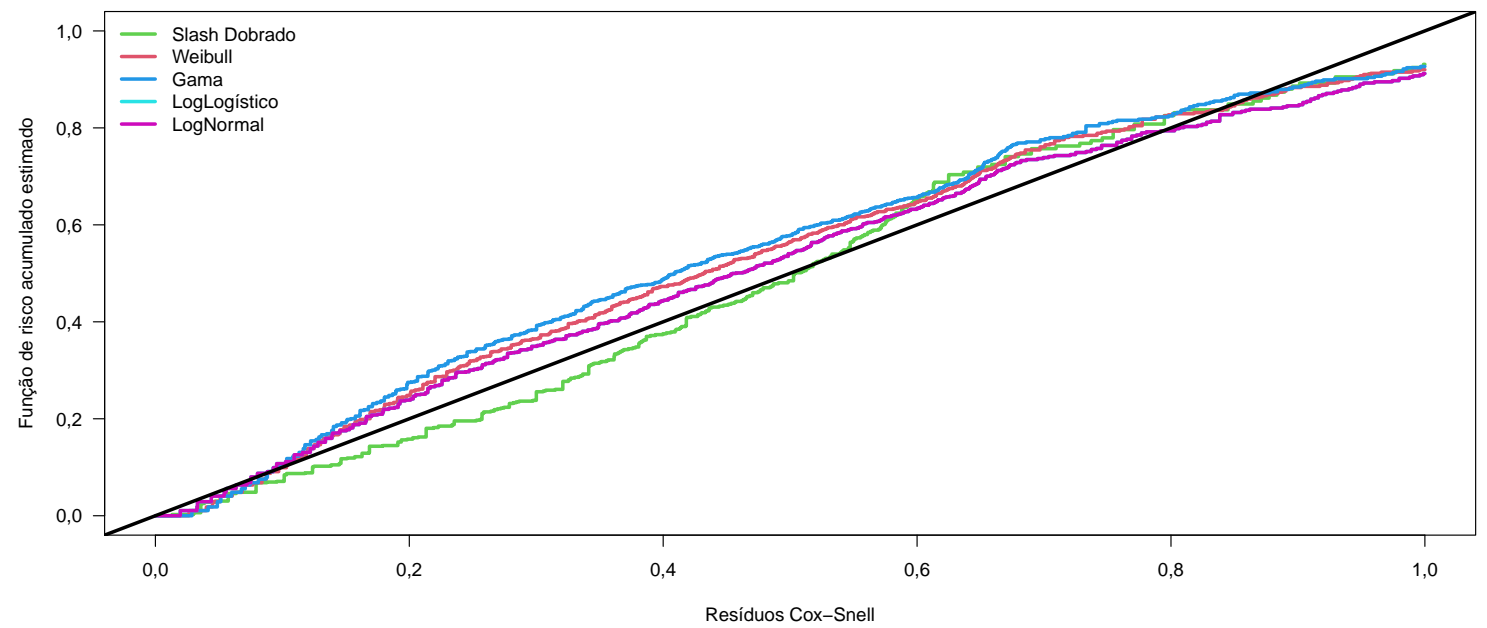

Figura 5.2: Resíduos Cox-Snell para o modelo Slash Dobrado e alguns modelos de mistura.

Na Tabela 5.3 encontram-se os valores de AIC (critério de informação de Akaike) para o modelo Slash Dobrado e para alguns modelos de mistura utilizados na literatura. Por este critério o modelo Slash Dobrado é melhor que os outros modelos.

Tabela 5.3: AIC dos modelos.

\begin{tabular}{lccccc}
\hline \hline Modelos & Slash Dobrado & Weibull & Gama & LogLogístico & LogNormal \\
\hline AIC & 6.188 & 6.849 & 6.874 & 6.824 & 6.802 \\
\hline \hline
\end{tabular}




\section{Capítulo 6}

\section{Considerações finais}

Após exaustivos estudos de simulação para analisar os estimadores dos parâmetros do modelo proposto. Sendo admitido casos com e sem heterogeneidade, diversos percentuais de censura e diferentes tamanhos amostrais. Além da implementação dos testes propostos, e uma aplicação aos dados do projeto ICESP chegou-se as seguintes conclusões.

\subsection{Conclusões}

Neste trabalho foi considerado um modelo que propõe tratar dados de longo termo sem ter a necessidade de separar os dados em grupos de curados e não curados, como é feito na literatura utilizando os modelos de mistura com fração de cura. O modelo se mostrou muito flexível, podendo tratar com altas concentrações de dados de longo termo. No modelo proposto, foi possível tratar dados com grande assimetria, além de grandes percentuais de censura.

Com um parâmetro que controla o peso da cauda da distribuição, pode-se verificar possíveis fatores que influenciam na existência de longo termo. Ou seja, pode-se estudar o que aumenta a probabilidade de um paciente ser de longo termo. E assim utilizar estas informações para tentar aumentar a sobrevida de pacientes.

Com os testes da razão de verossimilhanças e Wald pode-se verificar se o modelo Slash Dobrado é adequado aos dados e quais covariáveis deve-se adicionar.

$\mathrm{Na}$ análise dos dados que motivaram este trabalho verificou-se que o modelo Slash Dobrado foi mais adequado que os modelos comumente utilizados na literatura.

Este modelo é interessante para predizer o tempo de sobrevida em dados de longo termo, pois estima de forma satisfatória as curvas de sobrevida, tanto nos casos onde existem dados de longo termo quanto nos que não existem. 


\subsection{Trabalhos futuros}

Ao longo deste trabalho foram encontrados diversos obstáculos, alguns foram solucionados de forma satisfatória e outros ainda precisam de mais análises. Para superar os obstáculos foram surgindo várias ideias, algumas delas ficaram como propostas para trabalhos futuros. A seguir são mencionados alguns problemas sem solução que podem gerar trabalhos futuros.

O procedimento computacional possui forma complexa, no qual os valores iniciais podem fazer o procedimento demorar mais até convergir. Assim é necessário obter formas eficientes de atribuir valores iniciais.

Devido aos testes propostos terem hipóteses na fronteira do espaço paramétrico é necessário estudar melhor as propriedades assintóticas dos testes e a distribuição das estatísticas, assim como realizar estudos de simulação para verificar o poder dos testes. Ainda é necessário verificar se a propriedade de contiguidade é atendida.

Para os estimadores de máxima verossimilhança, pode-se ainda realizar estudos para verificar o comportamento das estimativas para grandes amostras, assim como propor intervalos de confiança, além dos testes pontuais já mencionados anteriormente.

Afim de reduzir erros computacionais é interessante verificar possíveis melhorias nas aproximações das integrais do procedimento utilizado, sendo interessante verificar procedimentos alternativos ao método de Newton Raphson.

A família de distribuições tratada neste trabalho possui forma bastante complexa o que inviabiliza a utilização prática. Desta forma é necessário a criação de pacotes computacionais que facilitem o acesso aos procedimentos utilizados.

Neste trabalho tínhamos a intenção de estudar heterogeneidade proveniente de efeitos aleatórios. Contudo devido a problemas computacionais, complexidade na dimensão das integrais, não foi possível concluir os estudos relativos a inclusão de efeitos aleatórios.

O modelo tratado neste trabalho possui diferentes parametrizações e formas de incluir covariáveis. Assim pode-se estudar o efeito de utilizar parametrizações diferentes na precisão dos procedimentos computacionais. Devido ao problema de identificabilidade ainda é possível verificar outras formas de corrigir esse problema e incluir covariáveis. 


\section{Apêndice A}

\section{Apêndice}

\section{A.1 Derivadas do modelo Normal Dobrado}

O logaritmo da função de verossimilhança do modelo é dado por

$$
l(\gamma)=-n \log (\sigma)+l_{1}+l_{2},
$$

em que

$$
l_{1}=\sum_{i=1}^{n} \delta_{i} \log \left(l_{1 i}\right), \quad l_{2}=\sum_{i=1}^{n}\left(1-\delta_{i}\right) \log \left(l_{2 i}\right)
$$

com

$$
\begin{aligned}
& l_{1 i}=\frac{e^{-\frac{\left(z_{i}-\mu\right)^{2}}{2 \sigma^{2}}}}{\sqrt{2 \pi}}+\frac{e^{-\frac{\left(z_{i}+\mu\right)^{2}}{2 \sigma^{2}}}}{\sqrt{2 \pi}}, \\
& l_{2 i}=\int_{z_{i}}^{\infty}\left(\frac{e^{-\frac{(y-\mu)^{2}}{2 \sigma^{2}}}}{\sqrt{2 \pi}}+\frac{e^{-\frac{(y+\mu)^{2}}{2 \sigma^{2}}}}{\sqrt{2 \pi}}\right) \mathrm{d} y .
\end{aligned}
$$

Nas derivadas da função $l_{1}$ aparece diretamente a função densidade de probabilidade da distribuição Normal. Será utilizada a função $\phi(y)=\frac{1}{\sqrt{(2 \pi)}} e^{-\frac{y^{2}}{2}}$. Assim $l_{1 i}$ fica da seguinte forma

$$
l_{1 i}=\phi\left(\frac{z_{i}-\mu}{\sigma}\right)+\phi\left(\frac{z_{i}+\mu}{\sigma}\right) .
$$

Já nas derivadas da função $l_{2}$, devido a integral envolvendo os tempos de censura, é necessário realizar transformações. Na função $l_{2 i}$, fazendo a mudança de variável $r=\frac{y \pm \mu}{\sigma}$, obtém-se 


$$
l_{2 i}=\sigma\left[S\left(\frac{z_{i}-\mu}{\sigma}\right)+S\left(\frac{z_{i}+\mu}{\sigma}\right)\right]
$$

$\operatorname{com} S(y)=\int_{y}^{\infty} \frac{1}{\sqrt{(2 \pi)}} e^{-\frac{u^{2}}{2}} \mathrm{~d} u$.

As expressões das primeiras derivadas da função $l_{1}$ em relação à $\gamma$, e em termos da função densidade de probabilidade da distribuição Normal, são dadas por

$$
\begin{aligned}
& \frac{\partial l_{1}}{\partial \mu}=\sum_{i=1}^{n} \delta_{i} \frac{\frac{e^{-\frac{\left(z_{i}-\mu\right)^{2}}{2 \sigma^{2}}}}{\sqrt{2 \pi}}\left(\frac{z_{i}-\mu}{\sigma^{2}}\right)-\frac{e^{-\frac{\left(z_{i}+\mu\right)^{2}}{2 \sigma^{2}}}}{\sqrt{2 \pi}}\left(\frac{z_{i}+\mu}{\sigma^{2}}\right)}{l_{1 i}}=\sum_{i=1}^{n} \delta_{i} \frac{\phi\left(\frac{z_{i}-\mu}{\sigma}\right)\left(\frac{z_{i}-\mu}{\sigma^{2}}\right)-\phi\left(\frac{z_{i}+\mu}{\sigma}\right)\left(\frac{z_{i}+\mu}{\sigma^{2}}\right)}{l_{1 i}}, \\
& \frac{\partial l_{1}}{\partial \sigma}=\sum_{i=1}^{n} \delta_{i} \frac{\frac{e^{-\frac{\left(z_{i}-\mu\right)^{2}}{2 \sigma^{2}}}}{\sqrt{2 \pi}}\left(\frac{\left(z_{i}-\mu\right)^{2}}{\sigma^{3}}\right)+\frac{e^{-\frac{\left(z_{i}+\mu\right)^{2}}{2 \sigma^{2}}}}{\sqrt{2 \pi}}\left(\frac{\left(z_{i}+\mu\right)^{2}}{\sigma^{3}}\right)}{l_{1 i}}=\sum_{i=1}^{n} \delta_{i} \frac{\phi\left(\frac{z_{i}-\mu}{\sigma}\right)\left(\frac{\left(z_{i}-\mu\right)^{2}}{\sigma^{3}}\right)+\phi\left(\frac{z_{i}+\mu}{\sigma}\right)\left(\frac{\left(z_{i}+\mu\right)^{2}}{\sigma^{3}}\right)}{l_{1 i}} .
\end{aligned}
$$

As segundas derivadas da função $l_{1}$ em relação à $\gamma$ são dadas por

$$
\begin{aligned}
& \frac{\partial^{2} l_{1}}{\partial \mu^{2}}=\sum_{i=1}^{n} \delta_{i} \frac{\frac{e^{-\frac{\left(z_{i}-\mu\right)^{2}}{2 \sigma^{2}}}}{\sqrt{2 \pi}}\left(\frac{\left(z_{i}-\mu\right)^{2}}{\sigma^{4}}-\frac{1}{\sigma^{2}}\right)+\frac{e^{-\frac{\left(z_{i}+\mu\right)^{2}}{2 \sigma^{2}}}}{\sqrt{2 \pi}}\left(\frac{\left(z_{i}+\mu\right)^{2}}{\sigma^{4}}-\frac{1}{\sigma^{2}}\right)}{l_{1 i}}-\left(\frac{\frac{\partial l_{1}}{\partial \mu}}{l_{1 i}}\right)^{2} \\
& =\sum_{i=1}^{n} \delta_{i} \frac{\phi\left(\frac{z_{i}-\mu}{\sigma}\right)\left(\frac{\left(z_{i}-\mu\right)^{2}}{\sigma^{4}}-\frac{1}{\sigma^{2}}\right)+\phi\left(\frac{z_{i}+\mu}{\sigma}\right)\left(\frac{\left(z_{i}+\mu\right)^{2}}{\sigma^{4}}-\frac{1}{\sigma^{2}}\right)}{l_{1 i}}-\left(\frac{\frac{\partial l_{1}}{\partial \mu}}{l_{1 i}}\right)^{2} \\
& \frac{\partial^{2} l_{1}}{\partial \mu \partial \sigma}=\sum_{i=1}^{n} \delta_{i} \frac{\frac{e^{-\frac{\left(z_{i}-\mu\right)^{2}}{2 \sigma^{2}}}}{\sqrt{2 \pi}}\left(\frac{\left(z_{i}-\mu\right)^{3}}{\sigma^{5}}-2 \frac{z_{i}-\mu}{\sigma^{3}}\right)-\frac{e^{-\frac{\left(z_{i}+\mu\right)^{2}}{2 \sigma^{2}}}}{\sqrt{2 \pi}}\left(\frac{\left(z_{i}+\mu\right)^{3}}{\sigma^{5}}-2 \frac{z_{i}+\mu}{\sigma^{3}}\right)}{l_{1 i}}-\frac{\frac{\partial l_{1}}{\partial \mu} \frac{\partial l_{1}}{\partial \sigma}}{l_{1 i}^{2}} \\
& =\sum_{i=1}^{n} \delta_{i} \frac{\phi\left(\frac{z_{i}-\mu}{\sigma}\right)\left(\frac{\left(z_{i}-\mu\right)^{3}}{\sigma^{5}}-2 \frac{z_{i}-\mu}{\sigma^{3}}\right)-\phi\left(\frac{z_{i}+\mu}{\sigma}\right)\left(\frac{\left(z_{i}+\mu\right)^{3}}{\sigma^{5}}-2 \frac{z_{i}+\mu}{\sigma^{3}}\right)}{l_{1 i}}-\frac{\frac{\partial l_{1}}{\partial \mu} \frac{\partial l_{1}}{\partial \sigma}}{l_{1 i}^{2}}, \\
& \frac{\partial^{2} l_{1}}{\partial \sigma^{2}}=\sum_{i=1}^{n} \delta_{i} \frac{\frac{e^{-\frac{\left(z_{i}-\mu\right)^{2}}{2 \sigma^{2}}}}{\sqrt{2 \pi}}\left(\frac{\left(z_{i}-\mu\right)^{4}}{\sigma^{6}}-3 \frac{\left(z_{i}-\mu\right)^{2}}{\sigma^{4}}\right)+\frac{e^{-\frac{\left(z_{i}+\mu\right)^{2}}{2 \sigma^{2}}}}{\sqrt{2 \pi}}\left(\frac{\left(z_{i}+\mu\right)^{4}}{\sigma^{6}}-3 \frac{\left(z_{i}+\mu\right)^{2}}{\sigma^{4}}\right)}{l_{1 i}}-\left(\frac{\frac{\partial l_{1}}{\partial \sigma}}{l_{1 i}}\right)^{2} \\
& =\sum_{i=1}^{n} \delta_{i} \frac{\phi\left(\frac{z_{i}-\mu}{\sigma}\right)\left(\frac{\left(z_{i}-\mu\right)^{4}}{\sigma^{6}}-3 \frac{\left(z_{i}-\mu\right)^{2}}{\sigma^{4}}\right)+\phi\left(\frac{z_{i}+\mu}{\sigma}\right)\left(\frac{\left(z_{i}+\mu\right)^{4}}{\sigma^{6}}-3 \frac{\left(z_{i}+\mu\right)^{2}}{\sigma^{4}}\right)}{l_{1 i}}-\left(\frac{\frac{\partial l_{1}}{\partial \sigma}}{l_{1 i}}\right)^{2} \text {. }
\end{aligned}
$$

A derivada da função $l_{2}$ em relação à $\mu$ é dada por

$$
\frac{\partial l_{2}}{\partial \mu}=\sum_{i=1}^{n}\left(1-\delta_{i}\right) \frac{\int_{z_{i}}^{\infty}\left[\frac{e^{-\frac{(y-\mu)^{2}}{2 \sigma^{2}}}}{\sqrt{2 \pi}}\left(\frac{y-\mu}{\sigma^{2}}\right)-\frac{e^{-\frac{(y+\mu)^{2}}{2 \sigma^{2}}}}{\sqrt{2 \pi}}\left(\frac{y+\mu}{\sigma^{2}}\right)\right] \mathrm{d} y}{l_{2 i}}
$$


Através da mudança de variável $r=\frac{(y \pm \mu)^{2}}{2 \sigma^{2}}$ e com algumas manipulações, obtém-se

$$
\frac{\partial l_{2}}{\partial \mu}=\sum_{i=1}^{n}\left(1-\delta_{i}\right) \frac{\phi\left(\frac{z_{i}-\mu}{\sigma}\right)-\phi\left(\frac{z_{i}+\mu}{\sigma}\right)}{l_{2 i}} .
$$

A derivada da função $l_{2}$ em relação à $\sigma$ é dada por

$$
\frac{\partial l_{2}}{\partial \sigma}=\sum_{i=1}^{n}\left(1-\delta_{i}\right) \frac{\int_{z_{i}}^{\infty}\left[\frac{e^{-\frac{(y-\mu)^{2}}{2 \sigma^{2}}}}{\sqrt{2 \pi}}\left(\frac{\left(z_{i}-\mu\right)^{2}}{\sigma^{3}}\right)+\frac{e^{-\frac{(y+\mu)^{2}}{2 \sigma^{2}}}}{\sqrt{2 \pi}}\left(\frac{\left(z_{i}+\mu\right)^{2}}{\sigma^{3}}\right)\right] \mathrm{d} y}{l_{2 i}}
$$

Com a mudança de variável $r=\frac{(y \pm \mu)^{2}}{2 \sigma^{2}}$ e com algumas manipulações, pode-se obter

$$
\frac{\partial l_{2}}{\partial \sigma}=\sum_{i=1}^{n}\left(1-\delta_{i}\right) \frac{1 / 2\left[S_{\Gamma}\left(\frac{\left(z_{i}-\mu\right)^{2}}{2 \sigma^{2}}, \frac{3}{2}, 1\right)+S_{\Gamma}\left(\frac{\left(z_{i}+\mu\right)^{2}}{2 \sigma^{2}}, \frac{3}{2}, 1\right)\right]}{l_{2 i}},
$$

sendo $S_{\Gamma}(y, a, b)=\int_{y}^{\infty} \frac{b^{a}}{\Gamma(a)} u^{a-1} e^{-b u} \mathrm{~d} u$ a função sobrevivência da distribuição Gama.

A derivada de $\frac{\partial l_{2}}{\partial \mu}$ em relação à $\mu$ é dada por

$$
\frac{\partial^{2} l_{2}}{\partial \mu^{2}}=\sum_{i=1}^{n}\left(1-\delta_{i}\right) \frac{\int_{z_{i}}^{\infty}\left[\frac{e^{-\frac{(y-\mu)^{2}}{2 \sigma^{2}}}}{\sqrt{2 \pi}}\left(\frac{(y-\mu)^{2}}{\sigma^{4}}-\frac{1}{\sigma^{2}}\right)+\frac{e^{-\frac{(y+\mu)^{2}}{2 \sigma^{2}}}}{\sqrt{2 \pi}}\left(\frac{(y+\mu)^{2}}{\sigma^{4}}-\frac{1}{\sigma^{2}}\right)\right] \mathrm{d} y}{l_{2 i}}+-\left(\frac{\frac{\partial l_{2}}{\partial \mu}}{l_{2 i}^{2}}\right)^{2} .
$$

Com a mudança de variável $r=\frac{(y \pm \mu)^{2}}{2 \sigma^{2}}$. Em seguida com integração por partes no primeiro termo. Pode-se obter após algumas manipulações

$$
\frac{\partial^{2} l_{2}}{\partial \mu^{2}}=\sum_{i=1}^{n}\left(1-\delta_{i}\right) \frac{\phi\left(\frac{z_{i}-\mu}{\sigma}\right)\left(\frac{z_{i}-\mu}{\sigma^{2}}\right)+\phi\left(\frac{z_{i}+\mu}{\sigma}\right)\left(\frac{z_{i}+\mu}{\sigma^{2}}\right)}{l_{2 i}}-\left(\frac{\frac{\partial l_{2}}{\partial \mu}}{l_{2 i}}\right)^{2} .
$$

A derivada de $\frac{\partial l_{2}}{\partial \mu}$ em relação à $\sigma$ é dada por 


$$
\frac{\partial^{2} l_{2}}{\partial \mu \partial \sigma}=\sum_{i=1}^{n}\left(1-\delta_{i}\right) \frac{\int_{z_{i}}^{\infty}\left[\frac{e^{-\frac{(y-\mu)^{2}}{2 \sigma^{2}}}}{\sqrt{2 \pi}}\left(\frac{(y-\mu)^{3}}{\sigma^{5}}-2 \frac{y-\mu}{\sigma^{3}}\right)-\frac{e^{-\frac{(y+\mu)^{2}}{2 \sigma^{2}}}}{\sqrt{2 \pi}}\left(\frac{(y+\mu)^{3}}{\sigma^{5}}-2 \frac{y+\mu}{\sigma^{3}}\right)\right] \mathrm{d} y}{l_{2 i}}-\frac{\frac{\partial l_{2}}{\partial \mu} \frac{\partial l_{2}}{\partial \sigma}}{l_{2 i}^{2}}
$$

Com a mudança de variável $r=\frac{(y \pm \mu)^{2}}{2 \sigma^{2}}$, em seguida utilizando integração por partes no primeiro termo, e com algumas manipulações, pode-se obter

$$
\frac{\partial^{2} l_{2}}{\partial \mu \partial \sigma}=\sum_{i=1}^{n}\left(1-\delta_{i}\right) \frac{\phi\left(\frac{z_{i}-\mu}{\sigma}\right)\left(\frac{\left(z_{i}-\mu\right)^{2}}{\sigma^{3}}\right)-\phi\left(\frac{z_{i}+\mu}{\sigma}\right)\left(\frac{\left(z_{i}+\mu\right)^{2}}{\sigma^{3}}\right)}{l_{2 i}}-\frac{\frac{\partial l_{2}}{\partial \mu} \frac{\partial l_{2}}{\partial \sigma}}{l_{2 i}^{2}}
$$

A derivada de $\frac{\partial l_{2}}{\partial \sigma}$ em relação à $\sigma$ é dada por

$$
\begin{aligned}
\frac{\partial^{2} l_{2}}{\partial \sigma^{2}} & =\sum_{i=1}^{n}\left(1-\delta_{i}\right) \frac{\int_{z_{i}}^{\infty}\left[\frac{e^{-\frac{(y-\mu)^{2}}{2 \sigma^{2}}}}{\sqrt{2 \pi}}\left(\frac{(y-\mu)^{4}}{\sigma^{6}}-3 \frac{(y-\mu)^{2}}{\sigma^{4}}\right)+\frac{e^{-\frac{(y+\mu)^{2}}{2 \sigma^{2}}}}{\sqrt{2 \pi}}\left(\frac{(y+\mu)^{4}}{\sigma^{6}}-3 \frac{(y+\mu)^{2}}{\sigma^{4}}\right)\right] \mathrm{d} y}{l_{2 i}} \\
& -\left(\frac{\frac{\partial l_{2}}{\partial \sigma}}{l_{2 i}}\right)^{2} .
\end{aligned}
$$

Com a mudança de variável $r=\frac{(y \pm \mu)^{2}}{2 \sigma^{2}}$, em seguida utilizando integração por partes no primeiro termo, e com algumas manipulações, pode-se obter

$$
\frac{\partial^{2} l_{2}}{\partial \sigma^{2}}=\sum_{i=1}^{n}\left(1-\delta_{i}\right) \frac{\phi\left(\frac{z_{i}-\mu}{\sigma}\right)\left(\frac{\left(z_{i}-\mu\right)^{3}}{\sigma^{4}}\right)+\phi\left(\frac{z_{i}+\mu}{\sigma}\right)\left(\frac{\left(z_{i}+\mu\right)^{3}}{\sigma^{4}}\right)}{l_{2 i}}-\left(\frac{\frac{\partial l_{2}}{\partial \sigma}}{l_{2 i}}\right)^{2}
$$




\section{A.2 Derivadas do modelo Slash Dobrado}

O logaritmo da função de verossimilhança do modelo é dado por

$$
l(\gamma)=n \log (q)-n \log (\sigma)+l_{1}+l_{2}
$$

em que

$$
l_{1}=\sum_{i=1}^{n} \delta_{i} \log \left(l_{1 i}\right), \quad l_{2}=\sum_{i=1}^{n}\left(1-\delta_{i}\right) \log \left(l_{2 i}\right)
$$

com

$$
\begin{aligned}
& l_{1 i}=\int_{0}^{1} u^{q}\left[\frac{e^{-\frac{\left(u z_{i}-\mu\right)^{2}}{2 \sigma^{2}}}}{\sqrt{2 \pi}}+\frac{e^{-\frac{\left(u z_{i}+\mu\right)^{2}}{2 \sigma^{2}}}}{\sqrt{2 \pi}}\right] \mathrm{d} u, \\
& l_{2 i}=\int_{0}^{1} u^{q} \int_{z_{i}}^{\infty}\left[\frac{e^{-\frac{(u y-\mu)^{2}}{2 \sigma^{2}}}}{\sqrt{2 \pi}}+\frac{e^{-\frac{(u y+\mu)^{2}}{2 \sigma^{2}}}}{\sqrt{2 \pi}}\right] \mathrm{d} y \mathrm{~d} u .
\end{aligned}
$$

Nas derivadas da função $l_{1}$ aparece diretamente a função densidade de probabilidade da distribuição Normal. Será utilizada a função $\phi(y)=\frac{1}{\sqrt{(2 \pi)}} e^{-\frac{y^{2}}{2}}$. Assim $l_{1 i}$ fica da seguinte forma

$$
l_{1 i}=\int_{0}^{1} u^{q}\left[\phi\left(\frac{u z_{i}-\mu}{\sigma}\right)+\phi\left(\frac{u z_{i}+\mu}{\sigma}\right)\right] \mathrm{d} u .
$$

Já nas derivadas da função $l_{2}$, devido a integral envolvendo os tempos de censura, é necessário realizar transformações. Na função $l_{2 i}$, fazendo a mudança de variável $r=\frac{u y \pm \mu}{\sigma}$, obtém-se

$$
l_{2 i}=\int_{0}^{1} u^{q-1} \sigma\left[S\left(\frac{u z_{i}-\mu}{\sigma}\right)+S\left(\frac{u z_{i}+\mu}{\sigma}\right)\right] \mathrm{d} u,
$$

$\operatorname{com} S(y)=\int_{y}^{\infty} \frac{1}{\sqrt{(} 2 \pi)} e^{-\frac{u^{2}}{2}} \mathrm{~d} u$

As expressões das primeiras derivadas da função $l_{1}$ em relação à $\gamma$, e em termos da função densidade de probabilidade da distribuição Normal, são dadas por 


$$
\begin{aligned}
\frac{\partial l_{1}}{\partial \mu} & =\sum_{i=1}^{n} \delta_{i} \frac{\int_{0}^{1} u^{q}\left[\frac{e^{-\frac{\left(u z_{i}-\mu\right)^{2}}{2 \sigma^{2}}}}{\sqrt{2 \pi}}\left(\frac{u z_{i}-\mu}{\sigma^{2}}\right)-\frac{e^{-\frac{\left(u z_{i}+\mu\right)^{2}}{2 \sigma^{2}}}}{\sqrt{2 \pi}}\left(\frac{u z_{i}+\mu}{\sigma^{2}}\right)\right] \mathrm{d} u}{l_{1 i}} \\
& =\sum_{i=1}^{n} \delta_{i} \frac{\int_{0}^{1} u^{q}\left[\phi\left(\frac{u z_{i}-\mu}{\sigma}\right)\left(\frac{u z_{i}-\mu}{\sigma^{2}}\right)-\phi\left(\frac{u z_{i}+\mu}{\sigma}\right)\left(\frac{u z_{i}+\mu}{\sigma^{2}}\right)\right] \mathrm{d} u}{l_{1 i}},
\end{aligned}
$$

$$
\begin{aligned}
\frac{\partial l_{1}}{\partial \sigma} & =\sum_{i=1}^{n} \delta_{i} \frac{\int_{0}^{1} u^{q}\left[\frac{e^{-\frac{\left(u z_{i}-\mu\right)^{2}}{2 \sigma^{2}}}}{\sqrt{2 \pi}}\left(\frac{\left(u z_{i}-\mu\right)^{2}}{\sigma^{3}}\right)+\frac{e^{-\frac{\left(u z_{i}+\mu\right)^{2}}{2 \sigma^{2}}}}{\sqrt{2 \pi}}\left(\frac{\left(u z_{i}+\mu\right)^{2}}{\sigma^{3}}\right)\right] \mathrm{d} u}{l_{1 i}} \\
& =\sum_{i=1}^{n} \delta_{i} \frac{\int_{0}^{1} u^{q}\left[\phi\left(\frac{u z_{i}-\mu}{\sigma}\right)\left(\frac{\left(u z_{i}-\mu\right)^{2}}{\sigma^{3}}\right)+\phi\left(\frac{u z_{i}+\mu}{\sigma}\right)\left(\frac{\left(u z_{i}+\mu\right)^{2}}{\sigma^{3}}\right)\right] \mathrm{d} u}{l_{1 i}}, \\
\frac{\partial l_{1}}{\partial q} & =\sum_{i=1}^{n} \delta_{i} \frac{\int_{0}^{1} u^{q} \log (u)\left[\frac{e^{-\frac{\left(u z_{i}-\mu\right)^{2}}{2 \sigma^{2}}}}{\sqrt{2 \pi}}+\frac{e^{-\frac{\left(u z_{i}+\mu\right)^{2}}{2 \sigma^{2}}}}{\sqrt{2 \pi}}\right] \mathrm{d} u}{l_{1 i}} \\
& =\sum_{i=1}^{n} \delta_{i} \frac{\int_{0}^{1} u^{q} \log (u)\left[\phi\left(\frac{u z_{i}-\mu}{\sigma}\right)+\phi\left(\frac{u z_{i}+\mu}{\sigma}\right)\right] \mathrm{d} u}{l_{1 i}} .
\end{aligned}
$$

As segundas derivadas da função $l_{1}$ em relação à $\gamma$ são dadas por

$$
\begin{aligned}
\frac{\partial^{2} l_{1}}{\partial \mu^{2}} & =\sum_{i=1}^{n} \delta_{i} \frac{\int_{0}^{1} u^{q}\left[\frac{e^{-\frac{\left(u z_{i}-\mu\right)^{2}}{2 \sigma^{2}}}}{\sqrt{2 \pi}}\left(\frac{\left(u z_{i}-\mu\right)^{2}}{\sigma^{4}}-\frac{1}{\sigma^{2}}\right)+\frac{e^{-\frac{\left(u z_{i}+\mu\right)^{2}}{2 \sigma^{2}}}}{\sqrt{2 \pi}}\left(\frac{\left(u z_{i}+\mu\right)^{2}}{\sigma^{4}}-\frac{1}{\sigma^{2}}\right)\right] \mathrm{d} u}{l_{1 i}}-\left(\frac{\frac{\partial l_{1}}{\partial \mu}}{l_{1 i}}\right)^{2} \\
& =\sum_{i=1}^{n} \delta_{i} \frac{\int_{0}^{1} u^{q}\left[\phi\left(\frac{u z_{i}-\mu}{\sigma}\right)\left(\frac{\left(u z_{i}-\mu\right)^{2}}{\sigma^{4}}-\frac{1}{\sigma^{2}}\right)+\phi\left(\frac{u z_{i}+\mu}{\sigma}\right)\left(\frac{\left(u z_{i}+\mu\right)^{2}}{\sigma^{4}}-\frac{1}{\sigma^{2}}\right)\right] \mathrm{d} u}{l_{1 i}}-\left(\frac{\frac{\partial l_{1}}{\partial \mu}}{l_{1 i}}\right)^{2}
\end{aligned}
$$




$$
\begin{aligned}
& \frac{\partial^{2} l_{1}}{\partial \mu \partial \sigma}=\sum_{i=1}^{n} \delta_{i} \frac{\int_{0}^{1} u^{q}\left[\frac{e^{-\frac{\left(u z_{i}-\mu\right)^{2}}{2 \sigma^{2}}}}{\sqrt{2 \pi}}\left(\frac{\left(u z_{i}-\mu\right)^{3}}{\sigma^{5}}-2 \frac{u z_{i}-\mu}{\sigma^{3}}\right)-\frac{e^{-\frac{\left(u z_{i}+\mu\right)^{2}}{2 \sigma^{2}}}}{\sqrt{2 \pi}}\left(\frac{\left(u z_{i}+\mu\right)^{3}}{\sigma^{5}}-2 \frac{u z_{i}+\mu}{\sigma^{3}}\right)\right] \mathrm{d} u}{l_{1 i}}-\frac{\frac{\partial l_{1}}{\partial \mu} \frac{\partial l_{1}}{\partial \sigma}}{l_{1 i}^{2}} \\
& =\sum_{i=1}^{n} \delta_{i} \frac{\int_{0}^{1} u^{q}\left[\phi\left(\frac{u z_{i}-\mu}{\sigma}\right)\left(\frac{\left(u z_{i}-\mu\right)^{3}}{\sigma^{5}}-2 \frac{u z_{i}-\mu}{\sigma^{3}}\right)-\phi\left(\frac{u z_{i}+\mu}{\sigma}\right)\left(\frac{\left(u z_{i}+\mu\right)^{3}}{\sigma^{5}}-2 \frac{u z_{i}+\mu}{\sigma^{3}}\right)\right] \mathrm{d} u}{l_{1 i}}-\frac{\frac{\partial l_{1}}{\partial \mu} \frac{\partial l_{1}}{\partial \sigma}}{l_{1 i}^{2}}, \\
& \frac{\partial^{2} l_{1}}{\partial \mu \partial q}=\sum_{i=1}^{n} \delta_{i} \frac{\int_{0}^{1} u^{q} \log (u)\left[\frac{e^{-\frac{\left(u z_{i}-\mu\right)^{2}}{2 \sigma^{2}}}}{\sqrt{2 \pi}}\left(\frac{u z_{i}-\mu}{\sigma^{2}}\right)-\frac{e^{-\frac{\left(u z_{i}+\mu\right)^{2}}{2 \sigma^{2}}}}{\sqrt{2 \pi}}\left(\frac{u z_{i}+\mu}{\sigma^{2}}\right)\right] \mathrm{d} u}{l_{1 i}}-\frac{\frac{\partial l_{1}}{\partial \mu} \frac{\partial l_{1}}{\partial q}}{l_{1 i}^{2}} \\
& =\sum_{i=1}^{n} \delta_{i} \frac{\int_{0}^{1} u^{q} \log (u)\left[\phi\left(\frac{u z_{i}-\mu}{\sigma}\right)\left(\frac{u z_{i}-\mu}{\sigma^{2}}\right)-\phi\left(\frac{u z_{i}+\mu}{\sigma}\right)\left(\frac{u z_{i}+\mu}{\sigma^{2}}\right)\right] \mathrm{d} u}{l_{1 i}}-\frac{\frac{\partial l_{1}}{\partial \mu} \frac{\partial l_{1}}{\partial q}}{l_{1 i}^{2}} \\
& \frac{\partial^{2} l_{1}}{\partial \sigma^{2}}=\sum_{i=1}^{n} \delta_{i} \frac{\int_{0}^{1} u^{q}\left[\frac{e^{-\frac{\left(u z_{i}-\mu\right)^{2}}{2 \sigma^{2}}}}{\sqrt{2 \pi}}\left(\frac{\left(u z_{i}-\mu\right)^{4}}{\sigma^{6}}-3 \frac{\left(u z_{i}-\mu\right)^{2}}{\sigma^{4}}\right)+\frac{e^{-\frac{\left(u z_{i}+\mu\right)^{2}}{2 \sigma^{2}}}}{\sqrt{2 \pi}}\left(\frac{\left(u z_{i}+\mu\right)^{4}}{\sigma^{6}}-3 \frac{\left(u z_{i}+\mu\right)^{2}}{\sigma^{4}}\right)\right] \mathrm{d} u}{l_{1 i}}-\left(\frac{\frac{\partial l_{1}}{\partial \sigma}}{l_{1 i}}\right)^{2} \\
& =\sum_{i=1}^{n} \delta_{i} \frac{\int_{0}^{1} u^{q}\left[\phi\left(\frac{u z_{i}-\mu}{\sigma}\right)\left(\frac{\left(u z_{i}-\mu\right)^{4}}{\sigma^{6}}-3 \frac{\left(u z_{i}-\mu\right)^{2}}{\sigma^{4}}\right)+\phi\left(\frac{u z_{i}+\mu}{\sigma}\right)\left(\frac{\left(u z_{i}+\mu\right)^{4}}{\sigma^{6}}-3 \frac{\left(u z_{i}+\mu\right)^{2}}{\sigma^{4}}\right)\right] \mathrm{d} u}{l_{1 i}}-\left(\frac{\frac{\partial l_{1}}{\partial \sigma}}{l_{1 i}}\right)^{2},
\end{aligned}
$$

$$
\begin{aligned}
& \frac{\partial^{2} l_{1}}{\partial \sigma \partial q}=\sum_{i=1}^{n} \delta_{i} \frac{\int_{0}^{1} u^{q} \log (u)\left[\frac{e^{-\frac{\left(u z_{i}-\mu\right)^{2}}{2 \sigma^{2}}}}{\sqrt{2 \pi}}\left(\frac{\left(u z_{i}-\mu\right)^{2}}{\sigma^{3}}\right)+\frac{e^{-\frac{\left(u z_{i}+\mu\right)^{2}}{2 \sigma^{2}}}}{\sqrt{2 \pi}}\left(\frac{\left(u z_{i}+\mu\right)^{2}}{\sigma^{3}}\right)\right] \mathrm{d} u}{l_{1 i}}-\frac{\frac{\partial l_{1}}{\partial \sigma} \frac{\partial l_{1}}{\partial q}}{l_{1 i}^{2}} \\
& =\sum_{i=1}^{n} \delta_{i} \frac{\int_{0}^{1} u^{q} \log (u)\left[\phi\left(\frac{u z_{i}-\mu}{\sigma}\right)\left(\frac{\left(u z_{i}-\mu\right)^{2}}{\sigma^{3}}\right)+\phi\left(\frac{u z_{i}+\mu}{\sigma}\right)\left(\frac{\left(u z_{i}+\mu\right)^{2}}{\sigma^{3}}\right)\right] \mathrm{d} u}{l_{1 i}}-\frac{\frac{\partial l_{1}}{\partial \sigma} \frac{\partial l_{1}}{\partial q}}{l_{1 i}^{2}}, \\
& \frac{\partial^{2} l_{1}}{\partial q^{2}}=\sum_{i=1}^{n} \delta_{i} \frac{\int_{0}^{1} u^{q}(\log (u))^{2}\left[\frac{e^{-\frac{\left(u z_{i}-\mu\right)^{2}}{2 \sigma^{2}}}}{\sqrt{2 \pi}}+\frac{e^{-\frac{\left(u z_{i}+\mu\right)^{2}}{2 \sigma^{2}}}}{\sqrt{2 \pi}}\right] \mathrm{d} u}{l_{1 i}}-\left(\frac{\frac{\partial l_{1}}{\partial q}}{l_{1 i}}\right)^{2} \\
& =\sum_{i=1}^{n} \delta_{i} \frac{\int_{0}^{1} u^{q}(\log (u))^{2}\left[\phi\left(\frac{u z_{i}-\mu}{\sigma}\right)+\phi\left(\frac{u z_{i}+\mu}{\sigma}\right)\right] \mathrm{d} u}{l_{1 i}}-\left(\frac{\frac{\partial l_{1}}{\partial q}}{l_{1 i}}\right)^{2} .
\end{aligned}
$$

A derivada da função $l_{2}$ em relação à $\mu$ é dada por

$$
\frac{\partial l_{2}}{\partial \mu}=\sum_{i=1}^{n}\left(1-\delta_{i}\right) \frac{\int_{0}^{1} \int_{z_{i}}^{\infty} u^{q}\left[\frac{e^{-\frac{(u y-\mu)^{2}}{2 \sigma^{2}}}}{\sqrt{2 \pi}}\left(\frac{u y-\mu}{\sigma^{2}}\right)-\frac{e^{-\frac{(u y+\mu)^{2}}{2 \sigma^{2}}}}{\sqrt{2 \pi}}\left(\frac{u y+\mu}{\sigma^{2}}\right)\right] \mathrm{d} y \mathrm{~d} u}{l_{2 i}}
$$


Através da mudança de variável $r=\frac{(u y \pm \mu)^{2}}{2 \sigma^{2}}$ e com algumas manipulações, obtém-se

$$
\frac{\partial l_{2}}{\partial \mu}=\sum_{i=1}^{n}\left(1-\delta_{i}\right) \frac{\int_{0}^{1} u^{q-1}\left[\phi\left(\frac{u z_{i}-\mu}{\sigma}\right)-\phi\left(\frac{u z_{i}+\mu}{\sigma}\right)\right] \mathrm{d} u}{l_{2 i}} .
$$

A derivada da função $l_{2}$ em relação à $\sigma$ é dada por

$$
\frac{\partial l_{2}}{\partial \sigma}=\sum_{i=1}^{n}\left(1-\delta_{i}\right) \frac{\int_{0}^{1} \int_{z_{i}}^{\infty} u^{q}\left[\frac{e^{-\frac{(u y-\mu)^{2}}{2 \sigma^{2}}}}{\sqrt{2 \pi}}\left(\frac{\left(u z_{i}-\mu\right)^{2}}{\sigma^{3}}\right)+\frac{e^{-\frac{(u y+\mu)^{2}}{2 \sigma^{2}}}}{\sqrt{2 \pi}}\left(\frac{\left(u z_{i}+\mu\right)^{2}}{\sigma^{3}}\right)\right] \mathrm{d} y \mathrm{~d} u}{l_{2 i}} .
$$

Com a mudança de variável $r=\frac{(u y \pm \mu)^{2}}{2 \sigma^{2}}$ e com algumas manipulações, pode-se obter

$$
\frac{\partial l_{2}}{\partial \sigma}=\sum_{i=1}^{n}\left(1-\delta_{i}\right) \frac{\int_{0}^{1} u^{q-1} / 2\left[S_{\Gamma}\left(\frac{\left(u z_{i}-\mu\right)^{2}}{2 \sigma^{2}}, \frac{3}{2}, 1\right)+S_{\Gamma}\left(\frac{\left(u z_{i}+\mu\right)^{2}}{2 \sigma^{2}}, \frac{3}{2}, 1\right)\right] \mathrm{d} u}{l_{2 i}},
$$

sendo $S_{\Gamma}(y, a, b)=\int_{y}^{\infty} \frac{b^{a}}{\Gamma(a)} u^{a-1} e^{-b u} \mathrm{~d} u$ a função sobrevivência da distribuição Gama.

A derivada da função $l_{2}$ em relação à $q$ é dada por

$$
\frac{\partial l_{2}}{\partial q}=\sum_{i=1}^{n}\left(1-\delta_{i}\right) \frac{\int_{0}^{1} \int_{z_{i}}^{\infty} u^{q} \log (u)\left[\frac{e^{-\frac{(u y-\mu)^{2}}{2 \sigma^{2}}}}{\sqrt{2 \pi}}+\frac{e^{-\frac{(u y+\mu)^{2}}{2 \sigma^{2}}}}{\sqrt{2 \pi}}\right] \mathrm{d} y \mathrm{~d} u}{l_{2 i}} .
$$

Com a mudança de variável $r=\frac{u y \pm \mu}{\sigma}$ e com algumas manipulações, pode-se obter

$$
\frac{\partial l_{2}}{\partial q}=\sum_{i=1}^{n}\left(1-\delta_{i}\right) \frac{\int_{0}^{1} \int_{z_{i}}^{\infty} u^{q-1} \log (u) \sigma\left[S\left(\frac{u z_{i}-\mu}{\sigma}\right)+S\left(\frac{u z_{i}+\mu}{\sigma}\right)\right] \mathrm{d} y \mathrm{~d} u}{l_{2 i}} .
$$

A derivada de $\frac{\partial l_{2}}{\partial \mu}$ em relação à $\mu$ é dada por 
$\frac{\partial^{2} l_{2}}{\partial \mu^{2}}=\sum_{i=1}^{n}\left(1-\delta_{i}\right) \frac{\int_{0}^{1} \int_{z_{i}}^{\infty} u^{q}\left[\frac{e^{-\frac{(u y-\mu)^{2}}{2 \sigma^{2}}}}{\sqrt{2 \pi}}\left(\frac{(u y-\mu)^{2}}{\sigma^{4}}-\frac{1}{\sigma^{2}}\right)+\frac{e^{-\frac{(u y+\mu)^{2}}{2 \sigma^{2}}}}{\sqrt{2 \pi}}\left(\frac{(u y+\mu)^{2}}{\sigma^{4}}-\frac{1}{\sigma^{2}}\right)\right] \mathrm{d} y \mathrm{~d} u}{l_{2 i}}+-\left(\frac{\frac{\partial l_{2}}{\partial \mu}}{l_{2 i}^{2}}\right)^{2}$.

Com a mudança de variável $r=\frac{(u y \pm \mu)^{2}}{2 \sigma^{2}}$. Em seguida com integração por partes no primeiro termo. Pode-se obter após algumas manipulações

$$
\frac{\partial^{2} l_{2}}{\partial \mu^{2}}=\sum_{i=1}^{n}\left(1-\delta_{i}\right) \frac{\int_{0}^{1} u^{q-1}\left[\phi\left(\frac{u z_{i}-\mu}{\sigma}\right)\left(\frac{u z_{i}-\mu}{\sigma^{2}}\right)+\phi\left(\frac{u z_{i}+\mu}{\sigma}\right)\left(\frac{u z_{i}+\mu}{\sigma^{2}}\right)\right] \mathrm{d} u}{l_{2 i}}-\left(\frac{\frac{\partial l_{2}}{\partial \mu}}{l_{2 i}}\right)^{2}
$$

A derivada de $\frac{\partial l_{2}}{\partial \mu}$ em relação à $\sigma$ é dada por

$\frac{\partial^{2} l_{2}}{\partial \mu \partial \sigma}=\sum_{i=1}^{n}\left(1-\delta_{i}\right) \frac{\int_{0}^{1} \int_{z_{i}}^{\infty} u^{q}\left[\frac{e^{-\frac{(u y-\mu)^{2}}{2 \sigma^{2}}}}{\sqrt{2 \pi}}\left(\frac{(u y-\mu)^{3}}{\sigma^{5}}-2 \frac{u y-\mu}{\sigma^{3}}\right)-\frac{e^{-\frac{(u y+\mu)^{2}}{2 \sigma^{2}}}}{\sqrt{2 \pi}}\left(\frac{(u y+\mu)^{3}}{\sigma^{5}}-2 \frac{u y+\mu}{\sigma^{3}}\right)\right] \mathrm{d} y \mathrm{~d} u}{l_{2 i}}-\frac{\frac{\partial l_{2}}{\partial \mu} \frac{\partial l_{2}}{\partial \sigma}}{l_{2 i}^{2}}$.

Com a mudança de variável $r=\frac{(u y \pm \mu)^{2}}{2 \sigma^{2}}$, em seguida utilizando integração por partes no primeiro termo, e com algumas manipulações, pode-se obter

$$
\frac{\partial^{2} l_{2}}{\partial \mu \partial \sigma}=\sum_{i=1}^{n}\left(1-\delta_{i}\right) \frac{\int_{0}^{1} u^{q-1}\left[\phi\left(\frac{u z_{i}-\mu}{\sigma}\right)\left(\frac{\left(u z_{i}-\mu\right)^{2}}{\sigma^{3}}\right)-\phi\left(\frac{u z_{i}+\mu}{\sigma}\right)\left(\frac{\left(u z_{i}+\mu\right)^{2}}{\sigma^{3}}\right)\right] \mathrm{d} u}{l_{2 i}}-\frac{\frac{\partial l_{2}}{\partial \mu} \frac{\partial l_{2}}{\partial \sigma}}{l_{2 i}^{2}}
$$

A derivada de $\frac{\partial l_{2}}{\partial \mu}$ em relação à $q$ é dada por

$$
\frac{\partial^{2} l_{2}}{\partial \mu \partial q}=\sum_{i=1}^{n}\left(1-\delta_{i}\right) \frac{\int_{0}^{1} \int_{z_{i}}^{\infty} u^{q} \log (u)\left[\frac{e^{-\frac{(u y-\mu)^{2}}{2 \sigma^{2}}}}{\sqrt{2 \pi}}\left(\frac{u y-\mu}{\sigma^{2}}\right)-\frac{e^{-\frac{(u y+\mu)^{2}}{2 \sigma^{2}}}}{\sqrt{2 \pi}}\left(\frac{u y+\mu}{\sigma^{2}}\right)\right] \mathrm{d} y \mathrm{~d} u}{l_{2 i}}-\frac{\frac{\partial l_{2}}{\partial \mu} \frac{\partial l_{2}}{\partial q}}{l_{2 i}^{2}} .
$$

Com a mudança de variável $r=\frac{(u y \pm \mu)^{2}}{2 \sigma^{2}}$, e com algumas manipulações, pode-se obter 


$$
\frac{\partial^{2} l_{2}}{\partial \mu \partial q}=\sum_{i=1}^{n}\left(1-\delta_{i}\right) \frac{\int_{0}^{1} u^{q-1} \log (u)\left[\phi\left(\frac{u z_{i}-\mu}{\sigma}\right)-\phi\left(\frac{u z_{i}+\mu}{\sigma}\right)\right] \mathrm{d} u}{l_{2 i}}-\frac{\frac{\partial l_{2}}{\partial \mu} \frac{\partial l_{2}}{\partial q}}{l_{2 i}^{2}} .
$$

A derivada de $\frac{\partial l_{2}}{\partial \sigma}$ em relação à $\sigma$ é dada por

$$
\begin{aligned}
\frac{\partial^{2} l_{2}}{\partial \sigma^{2}} & =\sum_{i=1}^{n}\left(1-\delta_{i}\right) \frac{\int_{0}^{1} \int_{z_{i}}^{\infty} u^{q}\left[\frac{e^{-\frac{(u y-\mu)^{2}}{2 \sigma^{2}}}}{\sqrt{2 \pi}}\left(\frac{(u y-\mu)^{4}}{\sigma^{6}}-3 \frac{(u y-\mu)^{2}}{\sigma^{4}}\right)+\frac{e^{-\frac{(u y+\mu)^{2}}{2 \sigma^{2}}}}{\sqrt{2 \pi}}\left(\frac{(u y+\mu)^{4}}{\sigma^{6}}-3 \frac{(u y+\mu)^{2}}{\sigma^{4}}\right)\right] \mathrm{d} y \mathrm{~d} u}{l_{2 i}} \\
& -\left(\frac{\frac{\partial l_{2}}{\partial \sigma}}{l_{2 i}}\right)^{2} .
\end{aligned}
$$

Com a mudança de variável $r=\frac{(u y \pm \mu)^{2}}{2 \sigma^{2}}$, em seguida utilizando integração por partes no primeiro termo, e com algumas manipulações, pode-se obter

$$
\frac{\partial^{2} l_{2}}{\partial \sigma^{2}}=\sum_{i=1}^{n}\left(1-\delta_{i}\right) \frac{\int_{0}^{1} u^{q-1}\left[\phi\left(\frac{u z_{i}-\mu}{\sigma}\right)\left(\frac{\left(u z_{i}-\mu\right)^{3}}{\sigma^{4}}\right)+\phi\left(\frac{u z_{i}+\mu}{\sigma}\right)\left(\frac{\left(u z_{i}+\mu\right)^{3}}{\sigma^{4}}\right)\right] \mathrm{d} u}{l_{2 i}}-\left(\frac{\frac{\partial l_{2}}{\partial \sigma}}{l_{2 i}}\right)^{2} .
$$

A derivada de $\frac{\partial l_{2}}{\partial \sigma}$ em relação à $q$ é dada por

$$
\frac{\partial^{2} l_{2}}{\partial \sigma \partial q}=\sum_{i=1}^{n}\left(1-\delta_{i}\right) \frac{\int_{0}^{1} \int_{z_{i}}^{\infty} u^{q} \log (u)\left[\frac{e^{-\frac{(u y-\mu)^{2}}{2 \sigma^{2}}}}{\sqrt{2 \pi}} \frac{(u y-\mu)^{2}}{\sigma^{3}}+\frac{e^{-\frac{(u y+\mu)^{2}}{2 \sigma^{2}}}}{\sqrt{2 \pi}} \frac{(u y+\mu)^{2}}{\sigma^{3}}\right] \mathrm{d} y_{\mathrm{d} u}}{l_{2 i}}-\frac{\frac{\partial l_{2}}{\partial \sigma} \frac{\partial l_{2}}{\partial q}}{l_{2 i}^{2}} .
$$

Com a mudança de variável $r=\frac{(u y \pm \mu)^{2}}{2 \sigma^{2}}$, e com algumas manipulações, pode-se obter

$$
\frac{\partial^{2} l_{2}}{\partial \sigma \partial q}=\sum_{i=1}^{n}\left(1-\delta_{i}\right) \frac{\int_{0}^{1} u^{q-1} \log (u) / 2\left[S_{\Gamma}\left(\frac{\left(u z_{i}-\mu\right)^{2}}{2 \sigma^{2}}, \frac{3}{2}, 1\right)+S_{\Gamma}\left(\frac{\left(u z_{i}+\mu\right)^{2}}{2 \sigma^{2}}, \frac{3}{2}, 1\right)\right] \mathrm{d} u}{l_{2 i}}-\frac{\frac{\partial l_{2}}{\partial \sigma} \frac{\partial l_{2}}{\partial \boldsymbol{\beta}_{q}}}{l_{2 i}^{2}}
$$

A derivada de $\frac{\partial l_{2}}{\partial q}$ em relação à $q$ é dada por 


$$
\frac{\partial^{2} l_{2}}{\partial q^{2}}=\sum_{i=1}^{n}\left(1-\delta_{i}\right) \frac{\int_{0}^{1} \int_{z_{i}}^{\infty} u^{q}(\log (u))^{2}\left[\frac{e^{-\frac{(u y-\mu)^{2}}{2 \sigma^{2}}}}{\sqrt{2 \pi}}+\frac{e^{-\frac{(u y+\mu)^{2}}{2 \sigma^{2}}}}{\sqrt{2 \pi}}\right] \mathrm{d} y \mathrm{~d} u}{l_{2 i}}-\left(\frac{\frac{\partial l_{2}}{\partial q}}{l_{2 i}}\right)^{2}
$$

Com a mudança de variável $r=\frac{u y \pm \mu}{\sigma}$, e com algumas manipulações, pode-se obter

$$
\frac{\partial^{2} l_{2}}{\partial q^{2}}=\sum_{i=1}^{n}\left(1-\delta_{i}\right) \frac{\int_{0}^{1} u^{q-1}\left(\log (u)^{2} \sigma\left[S\left(\frac{u z_{i}-\mu}{\sigma}\right)+S\left(\frac{u z_{i}+\mu}{\sigma}\right)\right] \mathrm{d} u\right.}{l_{2 i}}-\left(\frac{\frac{\partial l_{2}}{\partial q}}{l_{2 i}}\right)^{2} .
$$




\section{A.3 Derivadas do modelo Slash Dobrado com covariáveis}

O logaritmo da função de verossimilhança do modelo com covariáveis é dado por

$$
l(\gamma)=-n \log (\sigma)+\sum_{i=1}^{n} \boldsymbol{X}_{q i}^{T} \boldsymbol{\beta}_{q}+l_{1}+l_{2}
$$

em que

$$
l_{1}=\sum_{i=1}^{n} \delta_{i} \log \left(l_{1 i}\right), \quad l_{2}=\sum_{i=1}^{n}\left(1-\delta_{i}\right) \log \left(l_{2 i}\right)
$$

com

$$
\begin{aligned}
& l_{1 i}=\int_{0}^{1} u^{e^{\boldsymbol{X}_{q i}^{T} \boldsymbol{\beta}_{q}}}\left[\frac{e^{-\frac{\left(u z_{i}-e^{\left.\boldsymbol{X}_{\mu i}^{T} \boldsymbol{\beta}_{\mu}\right)^{2}}\right.}{2 \sigma^{2}}}}{\sqrt{2 \pi}}+\frac{e^{-\frac{\left(u z_{i}+e^{\left.\boldsymbol{X}_{\mu i}^{T} \boldsymbol{\beta}_{\mu}\right)^{2}}\right.}{2 \sigma^{2}}}}{\sqrt{2 \pi}}\right] \mathrm{d} u, \\
& l_{2 i}=\int_{0}^{1} u^{e^{\boldsymbol{X}_{q i}^{T} \boldsymbol{\beta}_{q}}} \int_{z_{i}}^{\infty}\left[\frac{e^{-\frac{\left(u y-e^{\left.\boldsymbol{X}_{\mu i}^{T} \boldsymbol{\beta}_{\mu}\right)^{2}}\right.}{2 \sigma^{2}}}}{\sqrt{2 \pi}}+\frac{e^{-\frac{\left(u y+e^{\left.\boldsymbol{X}_{\mu i}^{T} \boldsymbol{\beta}_{\mu}\right)^{2}}\right.}{2 \sigma^{2}}}}{\sqrt{2 \pi}}\right] \mathrm{d} y \mathrm{~d} u .
\end{aligned}
$$

Nas derivadas da função $l_{1}$ aparece diretamente a função densidade de probabilidade da distribuição Normal. Será utilizada a função $\phi(y)=\frac{1}{\sqrt{(2 \pi)}} e^{-\frac{y^{2}}{2}}$. Assim $l_{1 i}$ fica da seguinte forma

$$
l_{1 i}=\int_{0}^{1} u^{e^{\boldsymbol{X}_{q i}^{T} \boldsymbol{\beta}_{q}}}\left[\phi\left(\frac{u z_{i}-e^{\boldsymbol{X}_{\mu i}^{T} \boldsymbol{\beta}_{\mu}}}{\sigma}\right)+\phi\left(\frac{u z_{i}+e^{\boldsymbol{X}_{\mu i}^{T} \boldsymbol{\beta}_{\mu}}}{\sigma}\right)\right] \mathrm{d} u .
$$

Já nas derivadas da função $l_{2}$, devido a integral envolvendo os tempos de censura, é necessário realizar transformações. Na função $l_{2 i}$, fazendo a mudança de variável $r=\frac{u y \pm e^{\boldsymbol{X}_{\mu i}^{T} \boldsymbol{\beta}_{\mu}}}{\sigma}$, obtém-se

$$
l_{2 i}=\int_{0}^{1} u^{e^{\boldsymbol{X}_{q i}^{T} \boldsymbol{\beta}_{q}}-1} \sigma\left[S\left(\frac{u z_{i}-e^{\boldsymbol{X}_{\mu i}^{T} \boldsymbol{\beta}_{\mu}}}{\sigma}\right)+S\left(\frac{u z_{i}+e^{\boldsymbol{X}_{\mu i}^{T} \boldsymbol{\beta}_{\mu}}}{\sigma}\right)\right] \mathrm{d} u,
$$

$\operatorname{com} S(y)=\int_{y}^{\infty} \frac{1}{\sqrt{(2 \pi)}} e^{-\frac{u^{2}}{2}} \mathrm{~d} u$.

As expressões das primeiras derivadas da função $l_{1}$ em relação à $\gamma$, e em termos da função densidade de probabilidade da distribuição Normal, são dadas por

$\frac{\partial l_{1}}{\partial \boldsymbol{\beta}_{\mu}}=\sum_{i=1}^{n} \delta_{i} \frac{\int_{0}^{1} u^{e^{\boldsymbol{X}_{q i}^{T} \boldsymbol{\beta}_{q}}} e^{\boldsymbol{X}_{\mu i}^{T} \boldsymbol{\beta}_{\mu}} \boldsymbol{X}_{\mu i}\left[\frac{e^{-\frac{\left(u z_{i}-e^{\left.\boldsymbol{X}_{\mu i}^{T} \boldsymbol{\beta}_{\mu}\right)^{2}}\right.}{2 \sigma^{2}}}}{\sqrt{2 \pi}}\left(\frac{u z_{i}-e^{\boldsymbol{X}_{\mu i}^{T} \boldsymbol{\beta}_{\mu}}}{\sigma^{2}}\right)-\frac{e^{-\frac{\left(u z_{i}+e^{\left.\boldsymbol{X}_{\mu i}^{T} \boldsymbol{\beta}_{\mu}\right)^{2}}\right.}{2 \sigma^{2}}}}{\sqrt{2 \pi}}\left(\frac{u z_{i}+e^{\boldsymbol{X}_{\mu i}^{T} \boldsymbol{\beta}_{\mu}}}{\sigma^{2}}\right)\right] \mathrm{d} u}{l_{1 i}}$ 


$$
\begin{aligned}
& =\sum_{i=1}^{n} \delta_{i} \frac{\int_{0}^{1} u^{e^{\boldsymbol{X}_{q i}^{T} \boldsymbol{\beta}_{q}}} e^{\boldsymbol{X}_{\mu i}^{T} \boldsymbol{\beta}_{\mu}} \boldsymbol{X}_{\mu i}\left[\phi\left(\frac{u z_{i}-e^{\boldsymbol{X}_{\mu i}^{T} \boldsymbol{\beta}_{\mu}}}{\sigma}\right)\left(\frac{u z_{i}-e^{\boldsymbol{X}_{\mu i}^{T} \boldsymbol{\beta}_{\mu}}}{\sigma^{2}}\right)-\phi\left(\frac{u z_{i}+e^{\boldsymbol{X}_{\mu i}^{T} \boldsymbol{\beta}_{\mu}}}{\sigma}\right)\left(\frac{u z_{i}+e^{\boldsymbol{X}_{\mu i}^{T} \boldsymbol{\beta}_{\mu}}}{\sigma^{2}}\right)\right] \mathrm{d} u}{l_{1 i}}, \\
& \frac{\partial l_{1}}{\partial \sigma}=\sum_{i=1}^{n} \delta_{i} \frac{\int_{0}^{1} u^{e^{\boldsymbol{X}_{q i}^{T} \boldsymbol{\beta}_{q}}}\left[\frac{e^{-\frac{\left(u z_{i}-e^{\left.\boldsymbol{X}_{\mu i}^{T} \boldsymbol{\beta}_{\mu}\right)^{2}}\right.}{2 \sigma^{2}}}}{\sqrt{2 \pi}}\left(\frac{\left(u z_{i}-e^{\boldsymbol{X}_{\mu i}^{T} \boldsymbol{\beta}_{\mu}}\right)^{2}}{\sigma^{3}}\right)+\frac{e^{-\frac{\left(u z_{i}+e^{\left.\boldsymbol{X}_{\mu i}^{T} \boldsymbol{\beta}_{\mu}\right)^{2}}\right.}{2 \sigma^{2}}}}{\sqrt{2 \pi}}\left(\frac{\left(u z_{i}+e^{\boldsymbol{X}_{\mu i}^{T} \boldsymbol{\beta}_{\mu}}\right)^{2}}{\sigma^{3}}\right)\right] \mathrm{d} u}{l_{1 i}} \\
& =\sum_{i=1}^{n} \delta_{i} \frac{\int_{0}^{1} u^{e^{\boldsymbol{X}_{q i}^{T} \boldsymbol{\beta}_{q}}}\left[\phi\left(\frac{u z_{i}-e^{\boldsymbol{X}_{\mu i}^{T} \boldsymbol{\beta}_{\mu}}}{\sigma}\right)\left(\frac{\left(u z_{i}-e^{\boldsymbol{X}_{\mu i}^{T} \boldsymbol{\beta}_{\mu}}\right)^{2}}{\sigma^{3}}\right)+\phi\left(\frac{u z_{i}+e^{\boldsymbol{X}_{\mu i}^{T} \boldsymbol{\beta}_{\mu}}}{\sigma}\right)\left(\frac{\left(u z_{i}+e^{\boldsymbol{X}_{\mu i}^{T} \boldsymbol{\beta}_{\mu}}\right)^{2}}{\sigma^{3}}\right)\right] \mathrm{d} u}{l_{1 i}},
\end{aligned}
$$

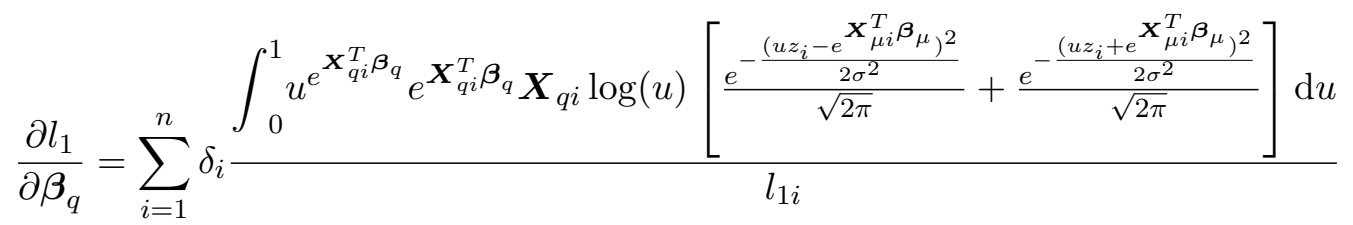

$$
\begin{aligned}
& =\sum_{i=1}^{n} \delta_{i} \frac{\int_{0}^{1} u^{e^{\boldsymbol{X}_{q i}^{T} \boldsymbol{\beta}_{q}}} e^{\boldsymbol{X}_{q i}^{T} \boldsymbol{\beta}_{q}} \boldsymbol{X}_{q i} \log (u)\left[\phi\left(\frac{u z_{i}-e^{\boldsymbol{X}_{\mu i}^{T} \boldsymbol{\beta}_{\mu}}}{\sigma}\right)+\phi\left(\frac{u z_{i}+e^{\boldsymbol{X}_{\mu i}^{T} \boldsymbol{\beta}_{\mu}}}{\sigma}\right)\right] \mathrm{d} u}{l_{1 i}} .
\end{aligned}
$$

As segundas derivadas da função $l_{1}$ em relação à $\boldsymbol{\gamma}$ são dadas por

$$
\begin{aligned}
& \frac{\partial^{2} l_{1}}{\partial \boldsymbol{\beta}_{\mu} \partial \boldsymbol{\beta}_{\mu}^{T}}=\sum_{i=1}^{n} \delta_{i} \frac{\int_{0}^{1} u^{e^{\boldsymbol{X}_{q i}^{T} \boldsymbol{\beta}_{q}}} e^{\boldsymbol{X}_{\mu i}^{T} \boldsymbol{\beta}_{\mu}} \boldsymbol{X}_{\mu i} \boldsymbol{X}_{\mu i}^{T} \frac{e^{-\frac{\left(u z_{i}-e^{\boldsymbol{X}_{\mu}^{T} \boldsymbol{\beta}_{\mu}}\right)^{2}}{2 \sigma^{2}}}}{\sqrt{2 \pi}}\left(\frac{\left(u z_{i}-e^{\boldsymbol{X}_{\mu i}^{T} \boldsymbol{\beta}_{\mu}}\right)^{2}}{\sigma^{4}} e^{\boldsymbol{X}_{\mu i}^{T} \boldsymbol{\beta}_{\mu}}-\frac{e^{\boldsymbol{X}_{\mu i}^{T} \boldsymbol{\beta}_{\mu}}}{\sigma^{2}}+\frac{u z_{i}-e^{\boldsymbol{X}_{\mu i}^{T} \boldsymbol{\beta}_{\mu}}}{\sigma^{2}}\right) \mathrm{d} u}{l_{1 i}}+ \\
& +\sum_{i=1}^{n} \delta_{i} \frac{\int_{0}^{1} u^{\boldsymbol{X}_{q i}^{T} \boldsymbol{\beta}_{q}} e^{\boldsymbol{X}_{\mu i}^{T} \boldsymbol{\beta}_{\mu}} \boldsymbol{X}_{\mu i} \boldsymbol{X}_{\mu i}^{T} \frac{e^{-\frac{\left(u z_{i}+e^{\left.\boldsymbol{X}_{\mu i}^{T} \boldsymbol{\beta}_{\mu}\right)^{2}}\right.}{2 \sigma^{2}}}}{\sqrt{2 \pi}}\left(\frac{\left(u z_{i}+e^{\boldsymbol{X}_{\mu i}^{T} \boldsymbol{\beta}_{\mu}}\right)^{2}}{\sigma^{4}} e^{\boldsymbol{X}_{\mu i}^{T} \boldsymbol{\beta}_{\mu}}-\frac{e^{\boldsymbol{X}_{\mu i}^{T} \boldsymbol{\beta}_{\mu}}}{\sigma^{2}}-\frac{u z_{i}+e^{\boldsymbol{X}_{\mu i}^{T} \boldsymbol{\beta}_{\mu}}}{\sigma^{2}}\right) \mathrm{d} u}{l_{1 i}}- \\
& -\frac{\frac{\partial l_{1}}{\partial \boldsymbol{\beta}_{\mu}}\left(\frac{\partial l_{1}}{\partial \boldsymbol{\beta}_{\mu}}\right)^{T}}{l_{1 i}^{2}} \\
& =\sum_{i=1}^{n} \delta_{i} \frac{\int_{0}^{1} u^{\boldsymbol{X}^{\boldsymbol{X}_{i i} \boldsymbol{\beta}_{q}}} e^{\boldsymbol{X}_{\mu i}^{T} \boldsymbol{\beta}_{\mu}} \boldsymbol{X}_{\mu i} \boldsymbol{X}_{\mu i}^{T} \phi\left(\frac{u z_{i}-e^{\mathbf{X}_{\mu i}^{T} \boldsymbol{\beta}_{\mu}}}{\sigma}\right)\left(\frac{\left(u z_{i}-e^{\boldsymbol{X}_{\mu i}^{T} \boldsymbol{\beta}_{\mu}}\right)^{2}}{\sigma^{4}} e^{\boldsymbol{X}_{\mu i}^{T} \boldsymbol{\beta}_{\mu}}-\frac{e^{\boldsymbol{X}_{\mu i}^{T} \boldsymbol{\beta}_{\mu}}}{\sigma^{2}}+\frac{u z_{i}-e^{\boldsymbol{X}_{\mu i}^{T} \boldsymbol{\beta}_{\mu}}}{\sigma^{2}}\right) \mathrm{d} u}{l_{1 i}}+ \\
& +\sum_{i=1}^{n} \delta_{i} \frac{\int_{0}^{1} u^{e^{\boldsymbol{X}_{q i}^{T} \boldsymbol{\beta}_{q}}} e^{\boldsymbol{X}_{\mu i}^{T} \boldsymbol{\beta}_{\mu}} \boldsymbol{X}_{\mu i} \boldsymbol{X}_{\mu i}^{T} \phi\left(\frac{u z_{i}+e^{\boldsymbol{X}_{\mu i}^{T} \boldsymbol{\beta}_{\mu}}}{\sigma}\right)\left(\frac{\left(u z_{i}+e^{\boldsymbol{X}_{\mu i}^{T} \boldsymbol{\beta}_{\mu}}\right)^{2}}{\sigma^{4}} e^{\boldsymbol{X}_{\mu i}^{T} \boldsymbol{\beta}_{\mu}}-\frac{e^{\boldsymbol{X}_{\mu i}^{T} \boldsymbol{\beta}_{\mu}}}{\sigma^{2}}-\frac{u z_{i}+e^{\boldsymbol{X}_{\mu i}^{T} \boldsymbol{\beta}_{\mu}}}{\sigma^{2}}\right) \mathrm{d} u}{l_{1 i}}- \\
& -\frac{\frac{\partial l_{1}}{\partial \boldsymbol{\beta}_{\mu}}\left(\frac{\partial l_{1}}{\partial \boldsymbol{\beta}_{\mu}}\right)^{T}}{l_{1 i}^{2}}
\end{aligned}
$$




$$
\begin{aligned}
& \frac{\partial^{2} l_{1}}{\partial \boldsymbol{\beta}_{\mu} \partial \sigma}=\sum_{i=1}^{n} \delta_{i} \frac{\int_{0}^{1} u^{e^{\boldsymbol{X}_{q i}^{T} \boldsymbol{\beta}_{q}}} e^{\boldsymbol{X}_{\mu i}^{T} \boldsymbol{\beta}_{\mu}} \boldsymbol{X}_{\mu i} \frac{e^{-\frac{\left(u z_{i}-e^{\left.\boldsymbol{X}_{\mu i}^{T} \boldsymbol{\beta}_{\mu}\right)^{2}}\right.}{2 \sigma^{2}}}}{\sqrt{2 \pi}}\left(\frac{\left(u z_{i}-e^{\boldsymbol{X}_{\mu i}^{T} \boldsymbol{\beta}_{\mu}}\right)^{3}}{\sigma^{5}}-2 \frac{u z_{i}-e^{\boldsymbol{X}_{\mu i}^{T} \boldsymbol{\beta}_{\mu}}}{\sigma^{3}}\right) \mathrm{d} u}{l_{1 i}}-
\end{aligned}
$$

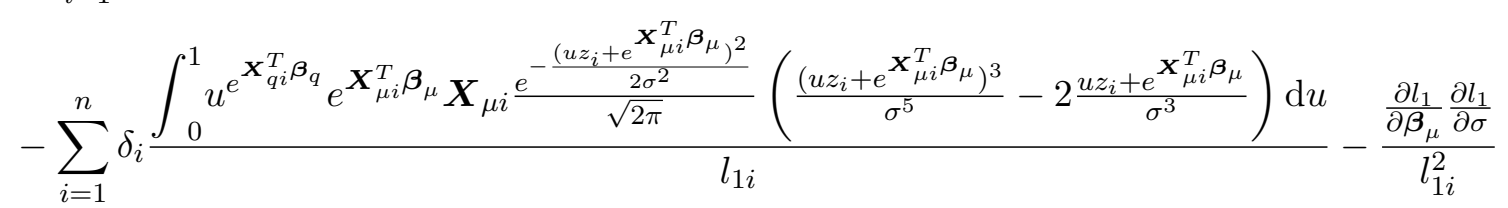

$$
\begin{aligned}
& =\sum_{i=1}^{n} \delta_{i} \frac{\int_{0}^{1} u^{e^{\boldsymbol{X}_{q i}^{T} \boldsymbol{\beta}_{q}}} e^{\boldsymbol{X}_{\mu i}^{T} \boldsymbol{\beta}_{\mu}} \boldsymbol{X}_{\mu i} \phi\left(\frac{u z_{i}-e^{\boldsymbol{X}_{\mu i}^{T} \boldsymbol{\beta}_{\mu}}}{\sigma}\right)\left(\frac{\left(u z_{i}-e^{\boldsymbol{X}_{\mu i}^{T} \boldsymbol{\beta}_{\mu}}\right)^{3}}{\sigma^{5}}-2 \frac{u z_{i}-e^{\boldsymbol{X}_{\mu i}^{T} \boldsymbol{\beta}_{\mu}}}{\sigma^{3}}\right) \mathrm{d} u}{l_{1 i}}- \\
& -\sum_{i=1}^{n} \delta_{i} \frac{\int_{0}^{1} u^{e^{\boldsymbol{X}_{q i}^{T} \boldsymbol{\beta}_{q}}} e^{\boldsymbol{X}_{\mu i}^{T} \boldsymbol{\beta}_{\mu}} \boldsymbol{X}_{\mu i} \phi\left(\frac{u z_{i}+e^{\boldsymbol{X}_{\mu i}^{T} \boldsymbol{\beta}_{\mu}}}{\sigma}\right)\left(\frac{\left(u z_{i}+e^{\boldsymbol{X}_{\mu i}^{T} \boldsymbol{\beta}_{\mu}}\right)^{3}}{\sigma^{5}}-2 \frac{u z_{i}+e^{\boldsymbol{X}_{\mu i}^{T} \boldsymbol{\beta}_{\mu}}}{\sigma^{3}}\right) \mathrm{d} u}{l_{1 i}}-\frac{\frac{\partial l_{1}}{\partial \boldsymbol{\beta}_{\mu}} \frac{\partial l_{1}}{\partial \sigma}}{l_{1 i}^{2}}, \\
& \frac{\partial^{2} l_{1}}{\partial \boldsymbol{\beta}_{\mu} \partial \boldsymbol{\beta}_{q}^{T}}=\sum_{i=1}^{n} \delta_{i} \frac{\int_{0}^{1} u^{e^{\boldsymbol{X}_{q i}^{T} \boldsymbol{\beta}_{q}}} e^{\boldsymbol{X}_{q i}^{T} \boldsymbol{\beta}_{q}} e^{\boldsymbol{X}_{\mu i}^{T} \boldsymbol{\beta}_{\mu}} \boldsymbol{X}_{\mu i} \boldsymbol{X}_{q i}^{T} \log (u) \frac{e^{-\frac{\left(u z_{i}-e^{\left.\boldsymbol{X}_{\mu i}^{T} \boldsymbol{\beta}_{\mu}\right)^{2}}\right.}{2 \sigma^{2}}}}{\sqrt{2 \pi}}\left(\frac{u z_{i}-e^{\boldsymbol{X}_{\mu i}^{T} \boldsymbol{\beta}_{\mu}}}{\sigma^{2}}\right) \mathrm{d} u}{l_{1 i}}- \\
& -\sum_{i=1}^{n} \delta_{i} \frac{\int_{0}^{1} u^{e^{\boldsymbol{X}_{q i}^{T} \boldsymbol{\beta}_{q}}} e^{\boldsymbol{X}_{q i}^{T} \boldsymbol{\beta}_{q}} e^{\boldsymbol{X}_{\mu i}^{T} \boldsymbol{\beta}_{\mu}} \boldsymbol{X}_{\mu i} \boldsymbol{X}_{q i}^{T} \log (u) \frac{e^{-\frac{\left(u z_{i}+e^{\left.\boldsymbol{X}_{\mu i}^{T} \boldsymbol{\beta}_{\mu}\right)^{2}}\right.}{2 \sigma^{2}}}}{\sqrt{2 \pi}}\left(\frac{u z_{i}+e^{\boldsymbol{X}_{\mu i}^{T} \boldsymbol{\beta}_{\mu}}}{\sigma^{2}}\right) \mathrm{d} u}{l_{1 i}}-\frac{\frac{\partial l_{1}}{\partial \boldsymbol{\beta}_{\mu}}\left(\frac{\partial l_{1}}{\partial \boldsymbol{\beta}_{q}}\right)^{T}}{l_{1 i}^{2}} \\
& =\sum_{i=1}^{n} \delta_{i} \frac{\int_{0}^{1} u^{e^{\boldsymbol{X}_{q i}^{T} \boldsymbol{\beta}_{q}}} e^{\boldsymbol{X}_{q i}^{T} \boldsymbol{\beta}_{q}} e^{\boldsymbol{X}_{\mu i}^{T} \boldsymbol{\beta}_{\mu}} \boldsymbol{X}_{\mu i} \boldsymbol{X}_{q i}^{T} \log (u) \phi\left(\frac{u z_{i}-e^{\boldsymbol{X}_{\mu i}^{T} \boldsymbol{\beta}_{\mu}}}{\sigma}\right)\left(\frac{u z_{i}-e^{\boldsymbol{X}_{\mu i}^{T} \boldsymbol{\beta}_{\mu}}}{\sigma^{2}}\right) \mathrm{d} u}{l_{1 i}}- \\
& -\sum_{i=1}^{n} \delta_{i} \frac{\int_{0}^{1} u^{e^{\boldsymbol{X}_{q i}^{T} \boldsymbol{\beta}_{q}}} e^{\boldsymbol{X}_{q i}^{T} \boldsymbol{\beta}_{q}} e^{\boldsymbol{X}_{\mu i}^{T} \boldsymbol{\beta}_{\mu}} \boldsymbol{X}_{\mu i} \boldsymbol{X}_{q i}^{T} \log (u) \phi\left(\frac{u z_{i}+e^{\boldsymbol{X}_{\mu i}^{T} \boldsymbol{\beta}_{\mu}}}{\sigma}\right)\left(\frac{u z_{i}+e^{\boldsymbol{X}_{\mu i}^{T} \boldsymbol{\beta}_{\mu}}}{\sigma^{2}}\right) \mathrm{d} u}{l_{1 i}}-\frac{\frac{\partial l_{1}}{\partial \boldsymbol{\beta}_{\mu}}\left(\frac{\partial l_{1}}{\partial \boldsymbol{\beta}_{q}}\right)^{T}}{l_{1 i}^{2}}
\end{aligned}
$$

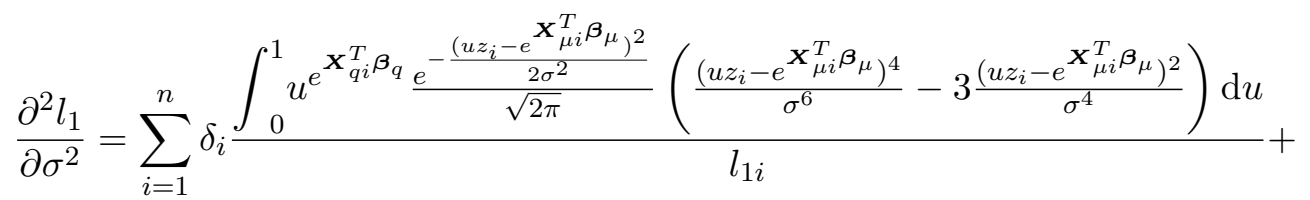

$$
\begin{aligned}
& +\sum_{i=1}^{n} \delta_{i} \frac{\int_{0}^{1} u^{e^{\mathbf{X}_{q i}^{T} \boldsymbol{\beta}_{q}}} \frac{e^{-\frac{\left(u z_{i}+e^{\left.\mathbf{X}_{\mu i}^{T} \boldsymbol{\beta}_{\mu}\right)^{2}}\right.}{2 \sigma^{2}}}}{\sqrt{2 \pi}}\left(\frac{\left(u z_{i}+e^{\left.\mathbf{X}_{\mu i}^{T} \boldsymbol{\beta}_{\mu}\right)^{4}}\right.}{\sigma^{6}}-3 \frac{\left(u z_{i}+e^{\left.\boldsymbol{X}_{\mu i}^{T} \boldsymbol{\beta}_{\mu}\right)^{2}}\right.}{\sigma^{4}}\right) \mathrm{d} u}{l_{1 i}}-\left(\frac{\frac{\partial l_{1}}{\partial \sigma}}{l_{1 i}}\right)^{2} \\
& =\sum_{i=1}^{n} \delta_{i} \frac{\int_{0}^{1} u^{e^{\boldsymbol{X}_{q i}^{T} \boldsymbol{\beta}_{q}}} \phi\left(\frac{u z_{i}-e^{\boldsymbol{X}_{\mu i}^{T} \boldsymbol{\beta}_{\mu}}}{\sigma}\right)\left(\frac{\left(u z_{i}-e^{\boldsymbol{X}_{\mu i}^{T} \boldsymbol{\beta}_{\mu}}\right)^{4}}{\sigma^{6}}-3 \frac{\left(u z_{i}-e^{\boldsymbol{X}_{\mu i}^{T} \boldsymbol{\beta}_{\mu}}\right)^{2}}{\sigma^{4}}\right) \mathrm{d} u}{l_{1 i}}+ \\
& +\sum_{i=1}^{n} \delta_{i} \frac{\int_{0}^{1} u^{e^{\boldsymbol{X}_{q i}^{T} \boldsymbol{\beta}_{q}}} \phi\left(\frac{u z_{i}+e^{\boldsymbol{X}_{\mu i}^{T} \boldsymbol{\beta}_{\mu}}}{\sigma}\right)\left(\frac{\left(u z_{i}+e^{\boldsymbol{X}_{\mu i}^{T} \boldsymbol{\beta}_{\mu}}\right)^{4}}{\sigma^{6}}-3 \frac{\left(u z_{i}+e^{\boldsymbol{X}_{\mu i}^{T} \boldsymbol{\beta}_{\mu}}\right)^{2}}{\sigma^{4}}\right) \mathrm{d} u}{l_{1 i}}-\left(\frac{\frac{\partial l_{1}}{\partial \sigma}}{l_{1 i}}\right)^{2},
\end{aligned}
$$




$$
\begin{aligned}
& \frac{\partial^{2} l_{1}}{\partial \sigma \partial \boldsymbol{\beta}_{q}}=\sum_{i=1}^{n} \delta_{i} \frac{\int_{0}^{1} u^{e^{\boldsymbol{X}_{q i}^{T} \boldsymbol{\beta}_{q}}} e^{\boldsymbol{X}_{q i}^{T} \boldsymbol{\beta}_{q}} \boldsymbol{X}_{q i} \log (u) \frac{e^{-\frac{\left(u z_{i}-e^{\left.\boldsymbol{X}_{\mu i}^{T} \boldsymbol{\beta}_{\mu}\right)^{2}}\right.}{2 \sigma^{2}}}}{\sqrt{2 \pi}}\left(\frac{\left(u z_{i}-e^{\left.\boldsymbol{X}_{\mu i}^{T} \boldsymbol{\beta}_{\mu}\right)^{2}}\right.}{\sigma^{3}}\right) \mathrm{d} u}{l_{1 i}}+ \\
& +\sum_{i=1}^{n} \delta_{i} \frac{\int_{0}^{1} u^{e^{\boldsymbol{X}_{q i}^{T} \boldsymbol{\beta}_{q}}} e^{\boldsymbol{X}_{q i}^{T} \boldsymbol{\beta}_{q}} \boldsymbol{X}_{q i} \log (u) \frac{e^{-\frac{\left(u z_{i}+e^{\left.\boldsymbol{X}_{\mu i}^{T} \boldsymbol{\beta}_{\mu}\right)^{2}}\right.}{2 \sigma^{2}}}}{\sqrt{2 \pi}}\left(\frac{\left(u z_{i}+e^{\left.\boldsymbol{X}_{\mu i}^{T} \boldsymbol{\beta}_{\mu}\right)^{2}}\right.}{\sigma^{3}}\right) \mathrm{d} u}{l_{1 i}}-\frac{\frac{\partial l_{1}}{\partial \sigma} \frac{\partial l_{1}}{\partial \boldsymbol{\beta}_{q}}}{l_{1 i}^{2}} \\
& =\sum_{i=1}^{n} \delta_{i} \frac{\int_{0}^{1} u^{e^{\boldsymbol{X}_{q i}^{T} \boldsymbol{\beta}_{q}}} e^{\boldsymbol{X}_{q i}^{T} \boldsymbol{\beta}_{q}} \boldsymbol{X}_{q i} \log (u) \phi\left(\frac{u z_{i}-e^{\boldsymbol{X}_{\mu i}^{T} \boldsymbol{\beta}_{\mu}}}{\sigma}\right)\left(\frac{\left(u z_{i}-e^{\boldsymbol{X}_{\mu i}^{T} \boldsymbol{\beta}_{\mu}}\right)^{2}}{\sigma^{3}}\right) \mathrm{d} u}{l_{1 i}}+ \\
& +\sum_{i=1}^{n} \delta_{i} \frac{\int_{0}^{1} u^{e^{\boldsymbol{X}_{q i}^{T} \boldsymbol{\beta}_{q}}} e^{\boldsymbol{X}_{q i}^{T} \boldsymbol{\beta}_{q}} \boldsymbol{X}_{q i} \log (u) \phi\left(\frac{u z_{i}+e^{\boldsymbol{X}_{\mu i}^{T} \boldsymbol{\beta}_{\mu}}}{\sigma}\right)\left(\frac{\left(u z_{i}+e^{\boldsymbol{X}_{\mu i}^{T} \boldsymbol{\beta}_{\mu}}\right)^{2}}{\sigma^{3}}\right) \mathrm{d} u}{l_{1 i}}-\frac{\frac{\partial l_{1}}{\partial \sigma} \frac{\partial l_{1}}{\partial \boldsymbol{\beta}_{q}}}{l_{1 i}^{2}},
\end{aligned}
$$

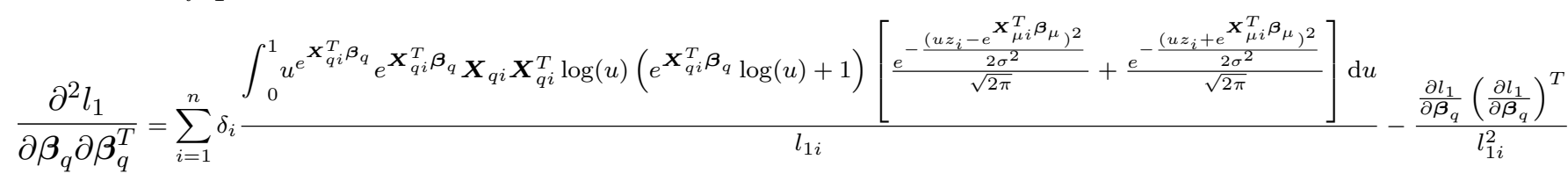

$$
\begin{aligned}
& =\sum_{i=1}^{n} \delta_{i} \frac{\int_{0}^{1} u^{e^{\mathbf{X}_{q i}^{T} \boldsymbol{\beta}_{q}}} e^{\boldsymbol{X}_{q i}^{T} \boldsymbol{\beta}_{q}} \boldsymbol{X}_{q i} \boldsymbol{X}_{q i}^{T} \log (u)\left(e^{\boldsymbol{X}_{q i}^{T} \boldsymbol{\beta}_{q}} \log (u)+1\right)\left[\phi\left(\frac{u z_{i}-e^{\boldsymbol{X}_{\mu i}^{T} \boldsymbol{\beta}_{\mu}}}{\sigma}\right)+\phi\left(\frac{u z_{i}+e^{\boldsymbol{X}_{\mu i}^{T} \boldsymbol{\beta}_{\mu}}}{\sigma}\right)\right] \mathrm{d} u}{l_{1 i}}-\frac{\frac{\partial \boldsymbol{l}_{1}}{\partial \boldsymbol{\beta}_{q}}\left(\frac{\partial l_{1}}{\partial \boldsymbol{\beta}_{q}}\right)^{T}}{l_{1 i}^{2}} .
\end{aligned}
$$

A derivada da função $l_{2}$ em relação à $\boldsymbol{\beta}_{\mu}$ é dada por

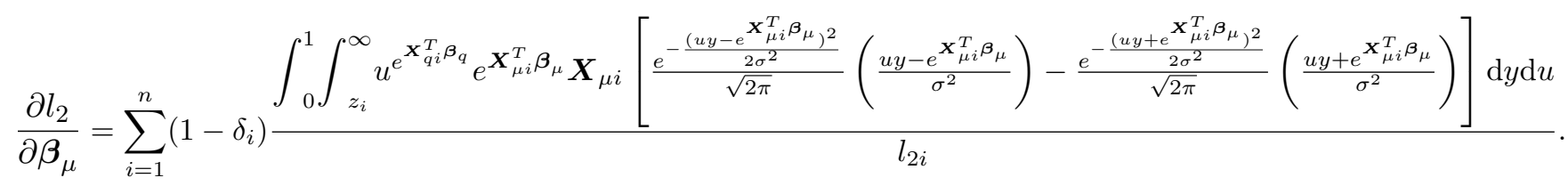

Através da mudança de variável $r=\frac{\left(u y \pm e^{\boldsymbol{X}_{\mu i}^{T} \boldsymbol{\beta}_{\mu}}\right)^{2}}{2 \sigma^{2}}$ e com algumas manipulações, obtém-se

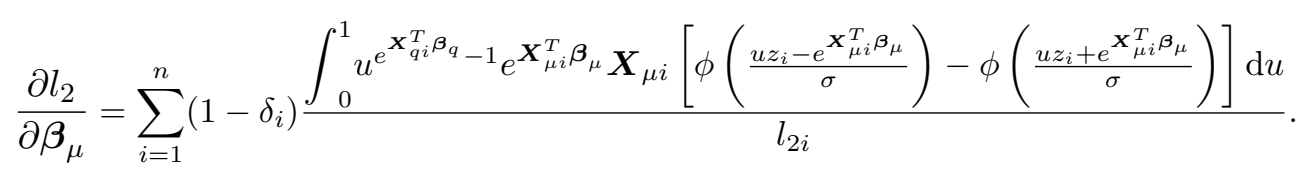

A derivada da função $l_{2}$ em relação à $\sigma$ é dada por

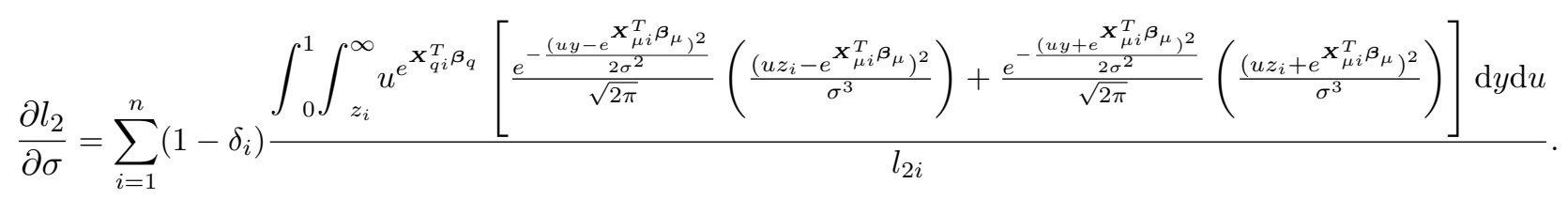


Com a mudança de variável $r=\frac{\left(u y \pm e^{\boldsymbol{X}_{\mu i}^{T} \boldsymbol{\beta}_{\mu}}\right)^{2}}{2 \sigma^{2}}$ e com algumas manipulações, pode-se obter

$$
\frac{\partial l_{2}}{\partial \sigma}=\sum_{i=1}^{n}\left(1-\delta_{i}\right) \frac{\int_{0}^{1} u^{e^{\mathbf{X}_{q i}^{T} \boldsymbol{\beta}_{q}}-1} / 2\left[S_{\Gamma}\left(\frac{\left(u z_{i}-e^{\boldsymbol{X}_{\mu i}^{T} \boldsymbol{\beta}_{\mu}}\right)^{2}}{2 \sigma^{2}}, \frac{3}{2}, 1\right)+S_{\Gamma}\left(\frac{\left(u z_{i}+e^{\boldsymbol{X}_{\mu i}^{T} \boldsymbol{\beta}_{\mu}}\right)^{2}}{2 \sigma^{2}}, \frac{3}{2}, 1\right)\right] \mathrm{d} u}{l_{2 i}},
$$

sendo $S_{\Gamma}(y, a, b)=\int_{y}^{\infty} \frac{b^{a}}{\Gamma(a)} u^{a-1} e^{-b u} \mathrm{~d} u$ a função sobrevivência da distribuição Gama.

A derivada da função $l_{2}$ em relação à $\boldsymbol{\beta}_{q}$ é dada por

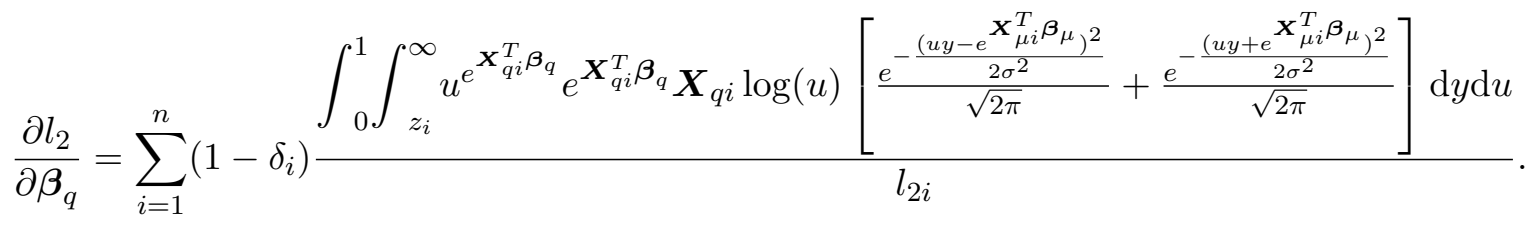

Com a mudança de variável $r=\frac{u y \pm e^{\boldsymbol{X}_{\mu i}^{T} \boldsymbol{\beta}_{\mu}}}{\sigma}$ e com algumas manipulações, pode-se obter

$\frac{\partial l_{2}}{\partial \boldsymbol{\beta}_{q}}=\sum_{i=1}^{n}\left(1-\delta_{i}\right) \frac{\int_{0}^{1} \int_{z_{i}}^{\infty} u^{e^{\boldsymbol{X}_{q i}^{T} \boldsymbol{\beta}_{q}}-1} e^{\boldsymbol{X}_{q i}^{T} \boldsymbol{\beta}_{q}} \boldsymbol{X}_{q i} \log (u) \sigma\left[S\left(\frac{u z_{i}-e^{\boldsymbol{X}_{\mu i}^{T} \boldsymbol{\beta}_{\mu}}}{\sigma}\right)+S\left(\frac{u z_{i}+e^{\boldsymbol{X}_{\mu i}^{T} \boldsymbol{\beta}_{\mu}}}{\sigma}\right)\right] \mathrm{d} y \mathrm{~d} u}{l_{2 i}}$.

A derivada de $\frac{\partial l_{2}}{\partial \boldsymbol{\beta}_{\mu}}$ em relação à $\boldsymbol{\beta}_{\mu}^{T}$ é dada por

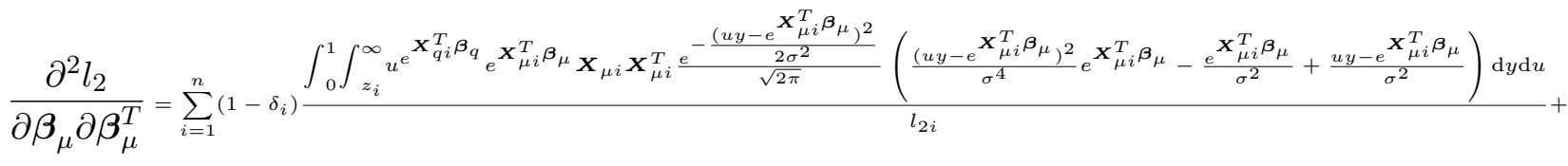

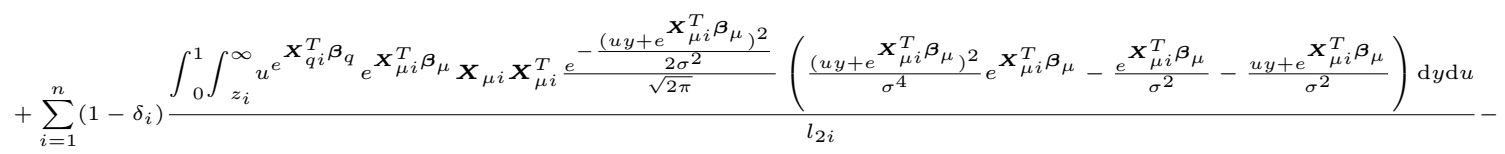

$$
\begin{aligned}
& -\frac{\frac{\partial l_{2}}{\partial \boldsymbol{\beta}_{\mu}}\left(\frac{\partial l_{2}}{\partial \boldsymbol{\beta}_{\mu}}\right)^{T}}{l_{2 i}^{2}} \text {. }
\end{aligned}
$$

Com as mudança de variáveis $r=\frac{\left(u y-e^{\boldsymbol{X}_{\mu i}^{T} \boldsymbol{\beta}_{\mu}}\right)^{2}}{2 \sigma^{2}}$ na primeira expressão e $r=\frac{\left(u y+e^{\boldsymbol{X}_{\mu i}^{T} \boldsymbol{\beta}_{\mu}}\right)^{2}}{2 \sigma^{2}}$ na segunda expressão. Em seguida com integração por partes no primeiro termo de cada expressão. Pode-se obter após algumas manipulações 


$$
\begin{aligned}
& \frac{\partial^{2} l_{2}}{\partial \boldsymbol{\beta}_{\mu} \partial \boldsymbol{\beta}_{\mu}^{T}}=\sum_{i=1}^{n}\left(1-\delta_{i}\right) \frac{\int_{0}^{1} u^{e^{\boldsymbol{X}_{q i}^{T} \boldsymbol{\beta}_{q}}-1} e^{\boldsymbol{X}_{\mu i}^{T} \boldsymbol{\beta}_{\mu}} \boldsymbol{X}_{\mu i} \boldsymbol{X}_{\mu i}^{T} \phi\left(\frac{u z_{i}-e^{\mathbf{X}_{\mu i}^{T} \boldsymbol{\beta}_{\mu}}}{\sigma}\right)\left(\frac{u z_{i}-e^{\mathbf{X}_{\mu i}^{T} \boldsymbol{\beta}_{\mu}}}{\sigma^{2}} e^{\boldsymbol{X}_{\mu i}^{T} \boldsymbol{\beta}_{\mu}}+1\right) \mathrm{d} u}{l_{2 i}}+
\end{aligned}
$$

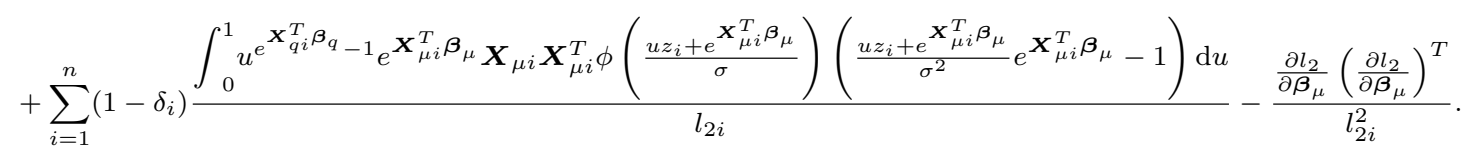

A derivada de $\frac{\partial l_{2}}{\partial \boldsymbol{\beta}_{\mu}}$ em relação à $\sigma$ é dada por

$$
\begin{aligned}
& \frac{\partial^{2} l_{2}}{\partial \boldsymbol{\beta}_{\mu} \partial \sigma}=\sum_{i=1}^{n}\left(1-\delta_{i}\right) \frac{\int_{0}^{1} \int_{z_{i}}^{\infty} u^{e^{\boldsymbol{X}_{q i}^{T} \boldsymbol{\beta}_{q}}} e^{\boldsymbol{X}_{\mu i}^{T} \boldsymbol{\beta}_{\mu}} \boldsymbol{X}_{\mu i} \frac{e^{-\frac{(u y-e}{\left.\boldsymbol{X}_{\mu i}^{T} \boldsymbol{\beta}_{\mu}\right)^{2}}}}{\sqrt{2 \pi}}\left(\frac{\left(u y-e^{\boldsymbol{X}_{\mu i}^{T} \boldsymbol{\beta}_{\mu}}\right)^{3}}{\sigma^{5}}-2 \frac{u y-e^{\boldsymbol{X}_{\mu i}^{T} \boldsymbol{\beta}_{\mu}}}{\sigma^{3}}\right) \mathrm{d} y \mathrm{~d} u}{l_{2 i}}-
\end{aligned}
$$

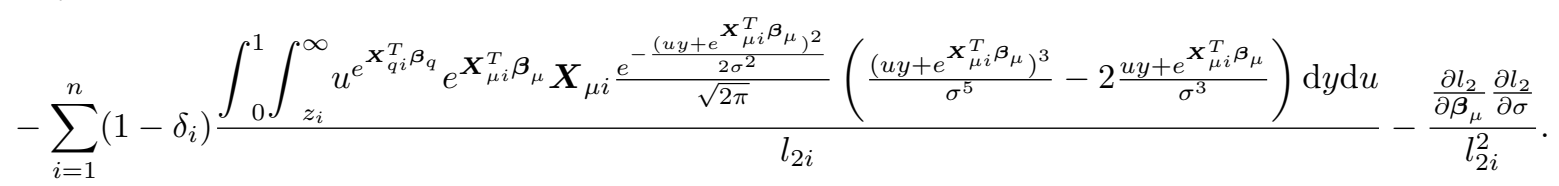

Com a mudança de variável $r=\frac{\left(u y \pm e^{\boldsymbol{X}_{\mu i}^{T} \boldsymbol{\beta}_{\mu}}\right)^{2}}{2 \sigma^{2}}$, em seguida utilizando integração por partes no primeiro termo, e com algumas manipulações, pode-se obter

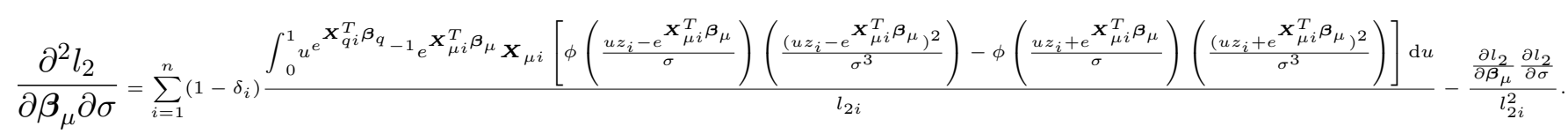

A derivada de $\frac{\partial l_{2}}{\partial \boldsymbol{\beta}_{\mu}}$ em relação à $\boldsymbol{\beta}_{q}^{T}$ é dada por

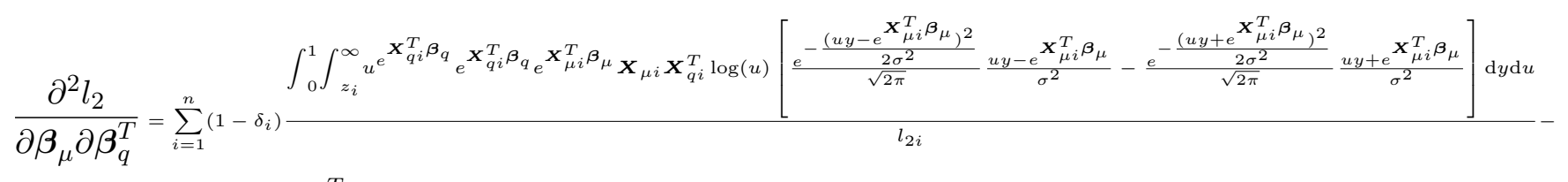

$$
\begin{aligned}
& -\frac{\frac{\partial l_{2}}{\partial \boldsymbol{\beta}_{\mu}}\left(\frac{\partial l_{2}}{\partial \boldsymbol{\beta}_{q}}\right)^{T}}{l_{2 i}^{2}} .
\end{aligned}
$$

Com a mudança de variável $r=\frac{\left(u y \pm e^{\boldsymbol{X}_{\mu i}^{T} \boldsymbol{\beta}_{\mu}}\right)^{2}}{2 \sigma^{2}}$, e com algumas manipulações, pode-se obter $\frac{\partial^{2} l_{2}}{\partial \boldsymbol{\beta}_{\mu} \partial \boldsymbol{\beta}_{q}^{T}}=\sum_{i=1}^{n}\left(1-\delta_{i}\right) \frac{\int_{0}^{1} u^{e^{\boldsymbol{X}_{q i}^{T} \boldsymbol{\beta}_{q}}-1} e^{\boldsymbol{X}_{q i}^{T} \boldsymbol{\beta}_{q}} \boldsymbol{X}_{\mu i} \boldsymbol{X}_{q i}^{T} \log (u)\left[\phi\left(\frac{u z_{i}-e^{\mathbf{X}_{\mu i}^{T} \boldsymbol{\beta}_{\mu}}}{\sigma}\right)-\phi\left(\frac{u z_{i}+e^{\mathbf{X}_{\mu i}^{T} \boldsymbol{\beta}_{\mu}}}{\sigma}\right)\right] \mathrm{d} u}{l_{2 i}}-\frac{\frac{\partial l_{2}}{\partial \boldsymbol{\beta}_{\mu}}\left(\frac{\partial l_{2}}{\partial \boldsymbol{\beta}_{q}}\right)^{T}}{l_{2 i}^{2}}$. 
A derivada de $\frac{\partial l_{2}}{\partial \sigma}$ em relação à $\sigma$ é dada por

$$
\begin{aligned}
& \frac{\partial^{2} l_{2}}{\partial \sigma^{2}}=\sum_{i=1}^{n}\left(1-\delta_{i}\right) \frac{\int_{0}^{1} \int_{z_{i}}^{\infty} u^{e^{\boldsymbol{X}_{q i}^{T} \boldsymbol{\beta}_{q}}} \frac{e^{-\frac{\left(u y-e^{\left.\boldsymbol{X}_{i j}^{T} \boldsymbol{\beta}_{\mu}\right)^{2}}\right.}{2 \sigma^{2}}}}{\sqrt{2 \pi}}\left(\frac{\left(u y-e^{\left.\boldsymbol{X}_{\mu i}^{T} \boldsymbol{\beta}_{\mu}\right)^{4}}\right.}{\sigma^{6}}-3 \frac{\left(u y-e^{\boldsymbol{X}_{\mu i}^{T} \boldsymbol{\beta}_{\mu}}\right)^{2}}{\sigma^{4}}\right) \mathrm{d} y \mathrm{~d} u}{l_{2 i}}+ \\
& +\sum_{i=1}^{n}\left(1-\delta_{i}\right) \frac{\int_{0}^{1} \int_{z_{i}}^{\infty} u^{e^{\boldsymbol{X}_{q i}^{T} \boldsymbol{\beta}_{q}}} \frac{e^{-\frac{\left(u y+e^{\left.\boldsymbol{X}_{\mu i}^{T} \boldsymbol{\beta}_{\mu}\right)^{2}}\right.}{2 \sigma^{2}}}}{\sqrt{2 \pi}}\left(\frac{\left(u y+e^{\boldsymbol{X}_{\mu i}^{T} \boldsymbol{\beta}_{\mu}}\right)^{4}}{\sigma^{6}}-3 \frac{\left(u y+e^{\left.\mathbf{X}_{\mu i}^{T} \boldsymbol{\beta}_{\mu}\right)^{2}}\right.}{\sigma^{4}}\right) \mathrm{d} y \mathrm{~d} u}{l_{2 i}}-\left(\frac{\frac{\partial l_{2}}{\partial \sigma}}{l_{2 i}}\right)^{2} .
\end{aligned}
$$

Com a mudança de variável $r=\frac{\left(u y \pm e^{\boldsymbol{X}_{\mu i}^{T} \boldsymbol{\beta}_{\mu}}\right)^{2}}{2 \sigma^{2}}$, em seguida utilizando integração por partes no primeiro termo, e com algumas manipulações, pode-se obter

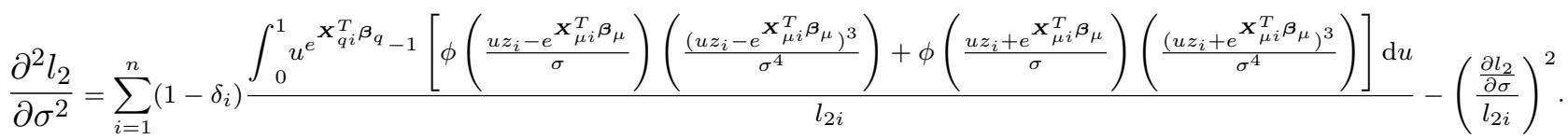

A derivada de $\frac{\partial l_{2}}{\partial \sigma}$ em relação à $\boldsymbol{\beta}_{q}$ é dada por

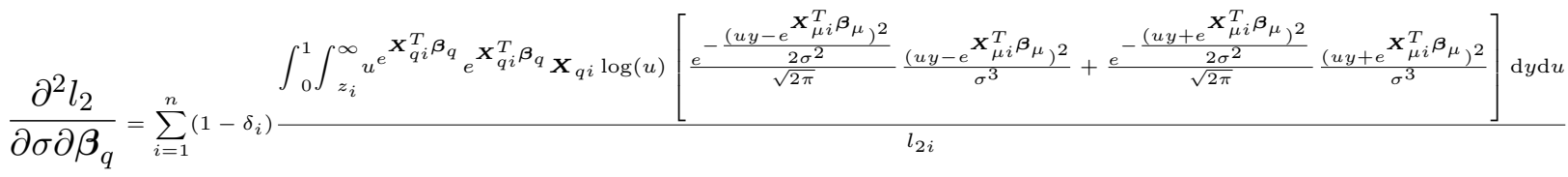

$$
\begin{aligned}
& -\frac{\frac{\partial l_{2}}{\partial \sigma} \frac{\partial l_{2}}{\partial \boldsymbol{\beta}_{q}}}{l_{2 i}^{2}} .
\end{aligned}
$$

Com a mudança de variável $r=\frac{\left(u y \pm e^{\boldsymbol{X}_{\mu i}^{T} \boldsymbol{\beta}_{\mu}}\right)^{2}}{2 \sigma^{2}}$, e com algumas manipulações, pode-se obter

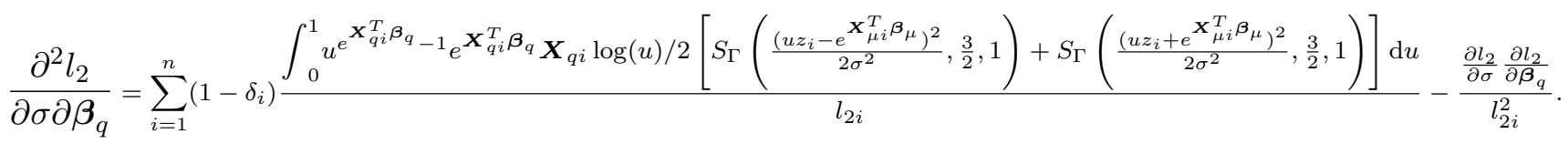

A derivada de $\frac{\partial l_{2}}{\partial \boldsymbol{\beta}_{q}}$ em relação à $\boldsymbol{\beta}_{q}^{T}$ é dada por 


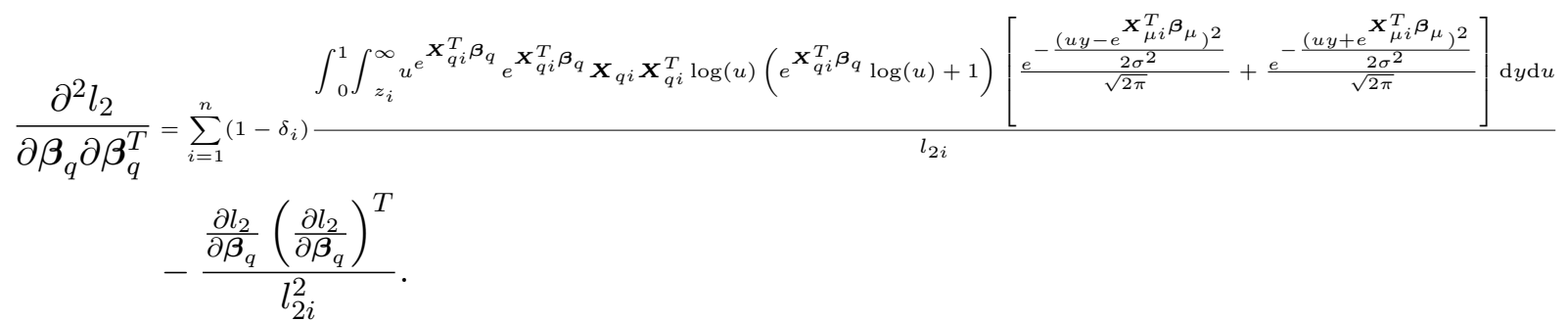

Com a mudança de variável $r=\frac{u y \pm e^{\boldsymbol{X}_{\mu i}^{T} \boldsymbol{\beta}_{\mu}}}{\sigma}$, e com algumas manipulações, pode-se obter

$\frac{\partial^{2} l_{2}}{\partial \boldsymbol{\beta}_{q} \partial \boldsymbol{\beta}_{q}^{T}}=\sum_{i=1}^{n}\left(1-\delta_{i}\right) \frac{\int_{0}^{1} u^{e^{\mathbf{X}_{q i}^{T} \boldsymbol{\beta}_{q}}-1} e^{\mathbf{X}_{q i}^{T} \boldsymbol{\beta}_{q}} \boldsymbol{X}_{q i} \boldsymbol{X}_{q i}^{T} \log (u) \sigma\left(e^{\mathbf{X}_{q i}^{T} \boldsymbol{\beta}_{q}} \log (u)+1\right)\left[S\left(\frac{u z_{i}-e^{\boldsymbol{X}_{\mu i}^{T} \boldsymbol{\beta}_{\mu}}}{\sigma}\right)+S\left(\frac{u z_{i}+e^{\boldsymbol{X}_{\mu i}^{T} \boldsymbol{\beta}_{\mu}}}{\sigma}\right)\right] \mathrm{d} u}{l_{2 i}}-\frac{\frac{\partial l_{2}}{\partial \boldsymbol{\beta}_{q}}\left(\frac{\partial l_{2}}{\partial \boldsymbol{\beta}_{q}}\right)^{T}}{l_{2 i}^{2}}$. 


\section{Referências Bibliográficas}

Berkson, J., \& Gage, R. P. 1952. The likelihood ratio, Wald, and Lagrange multiplier tests: an expository note. Journal of the American Statistical Association, 501-515. 1

Boag, J. W. 1949. Maximum likelihood estimates of the proportion of patients cured by cancer therapy. Journal of the Royal Statistical Society, 11, 15-44. 1

Broyden, Charles G. 1965. A class of methods for solving nonlinear simultaneous equations. Mathematics of computation, 19(92), 577-593. 20

Cavalcante, M. R. N. 2015. Robustecendo a distribuição normal. Mestrado em Estatıstica, Instituto de Matemática e Estatistica, Universidade de São Paulo. 11

Chen, M. H., Ibrahim, J. G., \& Sinha, D. A. 1999. A new baysian model for survival data with a surviving fraction. Journal of the American Statistical Association, 94, 909-919. 1

Cox, D. R., \& Snell, E. J. 1968. A General Definition of Residuals. Journal of the Royal Statistical Society. Series B (Methodological), 30(2), 248-275. 54

Gómez, Y.M, Gallardo D.I., \& De Castro, M. 2019. A regression model for positive data based on the slashed half-normal distribution. Revstat. In press. 11

Gui, Wenhao, Chen, Pei-Hua, \& Wu, Haiyan. 2013. A Folded Normal Slash Distribution and Its Applications to Non-negative Measurements. J. Data Sci., 11(01). 2, 11

Klein, J.P., \& Moeschberger, M.L. 2005. Survival Analysis: Techniques for Censored and Truncated Data. Statistics for Biology and Health. Springer New York. 17

Leone, F. C., Nelson, L. S., \& Nottingham, R. B. 1961. The Folded Normal Distribution. Technometrics, 3(4), 543-550. 7, 8 
Liu, Xuanyu, Tian, Guo-Liang, Fei, Yu, Shu, Lianjie, \& Zhao, Qiang. 2020. Folded normal regression models with applications in biomedicine. Journal of Computational and Applied Mathematics, 379, 112941. 15

Liu, Yucui, \& Kozubowski, T. 2015. A folded Laplace distribution. Journal of Statistical Distributions and Applications, 2, 1-17. 7

Locatelli, I., Rosina, A., Lichtenstein, P., \& Yashin, A. 2007. A correlated frailty model with longterm survivors for estimating the heritability of breast cancer. Statistics in medicine, $\mathbf{2 6} \mathbf{2 0}$, 3722-34. 1

Maller, Ross A., \& Zhou, Xian. 1996. Survival analysis with long-term survivors. Wiley Chichester, New York. 1

Nojoumizadeh, Maryam, \& Saberi, Zahra. 2019. A Folded Laplace Slash Distribution and Its Applications. International Journal of Applied Mathematics and Statistics, 20(02), 37-57. 11

Normilio-Silva, K., de Figueiredo, A. C., Pedroso-de Lima, A. C., Tunes-da Silva, G., da Silva, A. N., Levites, A. D. D., de Simone, A. T., Safra, P. L., Zancani, R., Tonini, P. C., et al. 2016. Long-term survival, quality of life, and quality-adjusted survival in critically ill patients with cancer. Critical care medicine, 44(7), 1327-1337. 2

Olmos, Neveka M., Varela, Héctor, Gómez, Héctor W., \& Bolfarine, Heleno. 2012. An extension of the half-normal distribution. Statistical Papers, 53, 875-886. 11

Pawitan, Yudi. 2001. In all likelihood : statistical modelling and inference using likelihood / Yudi Pawitan. Oxford science publications. Oxford New York: Clarendon Press Oxford University Press. 42

Pitkäniemi, Janne, Moltchanova, Elena, Haapala, Laura, Harjutsalo, Valma, Tuomilehto, Jaakko, \& Hakulinen, Timo. 2007. Genetic random effects model for family data with long-term survivors: analysis of diabetic nephropathy in Type 1 Diabetes. Genetic epidemiology, 31(11), 697-708. 1

Psarakis, S., \& Panaretoes, J. 1990. The folded t distribution. Communications in Statistics Theory and Methods, 19(7), 2717-2734. 7

Ribeiro, A.A., \& Karas, E.W. 2013. Otimizaçao continua: aspectos teóricos e computacionais. CENGAGE DO BRASIL. 20 
Rodrigues, J., Cancho, V. G., De Castro, M., \& Neto, F. L. 2009. On the unification of long-term survival models. Statistics and Probability Letters, 79(6), $753-759.1$

Self, Steven G, \& Liang, Kung-Yee. 1987. Asymptotic properties of maximum likelihood estimators and likelihood ratio tests under nonstandard conditions. Journal of the American Statistical Association, 82(398), 605-610. 35

Sen, Pranab K., \& Pedroso de Lima, Antonio C. 2011. Contiguity and irreconcilable nonstandard asymptotics of statistical tests. Brazilian Journal of Probability and Statistics, 25(3), $444-470$. 35

Tsagris, Michail, Beneki, Christina, \& Hassani, Hossein. 2014. On the Folded Normal Distribution. Mathematics, 2(1), 12-28. 9, 22

Wang, Jing, \& Genton, Marc G. 2006. The multivariate skew-slash distribution. Journal of Statistical Planning and Inference, 136(1), 209-220. 9, 11

Whelan, Simon, \& Goldman, Nick. 1999. Distributions of statistics used for the comparison of models of sequence evolution in phylogenetics. Molecular Biology and Evolution, 16(9), 12921292. 35

Wilks, S. S. 1962. Mathematical Statistics. New York, NY: Wiley. 35

Yakolev, A. Y., \& Tsodikov, A. D. 1996. Stochastic Models of Tumor Latency and Their Biostatistical Applications. World Scientific, New Jersey. 1 